\title{
Higher derivative couplings and massive supergravity in three dimensions
}

\author{
Sergei M. Kuzenko, ${ }^{a}$ Joseph Novak ${ }^{a}$ and Gabriele Tartaglino-Mazzucchelli ${ }^{a, b}$ \\ ${ }^{a}$ School of Physics M013, The University of Western Australia, \\ 35 Stirling Highway, Crawley W.A. 6009, Australia \\ ${ }^{b}$ Instituut voor Theoretische Fysica, KU Leuven, \\ Celestijnenlaan 200D, B-3001 Leuven, Belgium \\ E-mail: sergei.kuzenko@uwa.edu.au, joseph.novak@uwa.edu.au, \\ gabriele.tartaglino-mazzucchelli@uwa.edu.au
}

ABSTRACT: We develop geometric superspace settings to construct arbitrary higher derivative couplings (including $R^{n}$ terms) in three-dimensional supergravity theories with $\mathcal{N} \leq 3$ by realising them as conformal supergravity coupled to certain compensators. For all known off-shell supergravity formulations, we construct supersymmetric invariants with up to and including four derivatives. As a warming-up exercise, we first give a new and completely geometric derivation of such invariants in $\mathcal{N}=1$ supergravity. Upon reduction to components, they agree with those given in arXiv:0907.4658 and arXiv:1005.3952. We then carry out a similar construction in the case of $\mathcal{N}=2$ supergravity for which there exist two minimal formulations that differ by the choice of compensating multiplet: (i) a chiral scalar multipet; (ii) a vector multiplet. For these formulations all four derivative invariants are constructed in completely general and gauge independent form. For a general supergravity model (in the $\mathcal{N}=1$ and minimal $\mathcal{N}=2$ cases) with curvature-squared and lower order terms, we derive the superfield equations of motion, linearise them about maximally supersymmetric backgrounds and obtain restrictions on the parameters that lead to models for massive supergravity. We use the non-minimal formulation for $\mathcal{N}=2$ supergravity (which corresponds to a complex linear compensator) to construct a novel consistent theory of massive supergravity. In the case of $\mathcal{N}=3$ supergravity, we employ the off-shell formulation with a vector multiplet as compensator to construct for the first time various higher derivative invariants. These invariants may be used to derive models for $\mathcal{N}=3$ massive supergravity. As a bi-product of our analysis, we also present superfield equations for massive higher spin multiplets in $(1,0),(1,1)$ and $(2,0)$ anti-de Sitter superspaces.

KEywords: Extended Supersymmetry, Superspaces, Supergravity Models

ARXIV EPRINT: 1506.09063 


\section{Contents}

1 Introduction 1

$2 \mathcal{N}=1$ supergravity models $\quad 3$

2.1 Conventional superspace 3

2.2 The superconformal setting $\quad 6$

$\begin{array}{ll}2.3 \text { Supergravity invariants } & 9\end{array}$

2.3.1 The supergravity action 9

2.3.2 The $S^{n}$ invariants 9

$\begin{array}{ll}\text { 2.3.3 The scalar curvature squared invariant } & 10\end{array}$

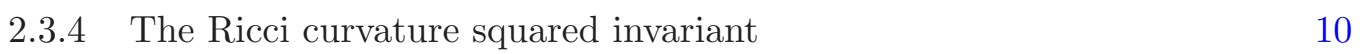

$\begin{array}{ll}2.4 \text { Models for massive supergravity } & 12\end{array}$

$3 \mathcal{N}=2$ supergravity models with a chiral compensator $\quad 16$

$\begin{array}{ll}3.1 \text { Conventional superspace } & 16\end{array}$

$\begin{array}{ll}3.2 & \text { Type I minimal supergravity in conventional superspace } \\ & 18\end{array}$

$\begin{array}{ll}\text { 3.3 The superconformal setting for type I supergravity } & 19\end{array}$

$\begin{array}{lll}3.4 & \text { Supergravity invariants } & 21\end{array}$

$\begin{array}{lll}3.4 .1 & \text { The supergravity action } & 22\end{array}$

$\begin{array}{ll}3.4 .2 & \text { The } R M^{n} \text { invariants }\end{array}$

$\begin{array}{lll}3.4 .3 & \text { The scalar curvature squared invariant } & 22\end{array}$

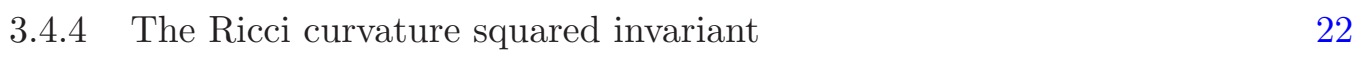

$\begin{array}{ll}3.5 & \text { Models for massive supergravity }\end{array}$

$4 \mathcal{N}=2$ supergravity models with a real linear compensator $\quad 28$

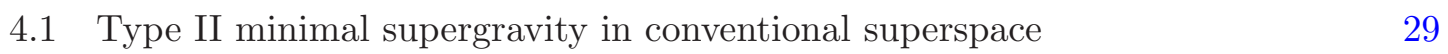

$\begin{array}{ll}4.2 \text { The superconformal setting } & 29\end{array}$

4.2.1 The real linear compensator $\quad 29$

4.2.2 The $B F$ action $\quad 32$

4.3 Supergravity invariants 33

$\begin{array}{lll}\text { 4.3.1 The supergravity action } & 33\end{array}$

$\begin{array}{lll}\text { 4.3.2 The } R h^{n} \text { invariants } & 33\end{array}$

4.3.3 The Ricci curvature squared invariant 34

4.4 Models for massive supergravity 35

$5 \mathcal{N}=2$ supergravity models with a complex linear compensator $\quad 39$

5.1 Non-minimal supergravity in conventional superspace 40

$\begin{array}{lll}5.2 & \text { The superconformal setting } & 42\end{array}$

$\begin{array}{lll}5.3 & \text { Massive supergravity } & 43\end{array}$ 
$6 \mathcal{N}=3$ supergravity with a compensating vector multiplet

6.1 Conventional superspace 44

$\begin{array}{lll}6.2 & \text { The superconformal setting } & 47\end{array}$

$\begin{array}{lll}\text { 6.2.1 The abelian vector multiplet } & 47\end{array}$

6.2.2 The $B F$ action $\quad 49$

$\begin{array}{lll}6.3 & \text { Supergravity invariants } & 50\end{array}$

$\begin{array}{lll}\text { 6.3.1 The supergravity action } & 50\end{array}$

6.3.2 The $R h^{n}$ invariants $\quad 51$

$\begin{array}{lll}\text { 6.3.3 Ricci squared invariants } & 52\end{array}$

7 Concluding comments $\quad 54$

$\begin{array}{ll}\text { A Geometry of conformal superspace } & \mathbf{5 7}\end{array}$

A.1 The $\mathcal{N}=1$ case $\quad 58$

$\begin{array}{ll}\text { A.2 The } \mathcal{N}=2 \text { case } & 59\end{array}$

$\begin{array}{ll}\text { A.3 The } \mathcal{N}=3 \text { case } & 60\end{array}$

B Results for $\mathcal{N}=1$ prepotential deformation $\quad 60$

C Results for $\mathcal{N}=2$ prepotential deformation $\quad 63$

\section{Introduction}

Higher-derivative gravity has attracted attention, on and off, for over half a century. Interest in such theories was spurred on in the early 1960s when it was noticed $[1,2]$ that the renormalization of divergences in quantum field theories in curved spacetime requires higher-derivative counterterms containing the curvature tensor squared. A decade later it was established [3] that adding the higher-derivative structures $R^{a b} R_{a b}$ and $R^{2}$ to the Einstein-Hilbert (EH) Lagrangian leads to a renormalizable theory in four spacetime dimensions, the price for renormalizability being unphysical ghost modes in the theory. Furthermore, an important development took place in 1980 when Starobinsky proposed his (nowadays famous) model of inflation [4] obtained by complementing the EH Lagrangian with a term proportional to the scalar curvature squared.

In three dimensions (3D), consistent models for massive gravity can be constructed by making use of certain higher-derivative extensions of the EH action. One such extension was proposed more than thirty years ago [5] and is known as topologically massive gravity (TMG). This model is obtained by adding a Lorentzian Chern-Simons term (which is cubic in derivatives of the gravitational field) to the $\mathrm{EH}$ action. The resulting theory does not preserve parity, is ghost-free and propagates a single massive state of helicity \pm 2 , where the sign depends on that of the Lorentz Chern-Simons term. More recently, a parity-preserving model for 3D massive gravity has been proposed [6] (see also [7]). It is obtained by combining the "wrong sign" EH Lagrangian with a fourth-order term $m^{-2}\left(R^{a b} R_{a b}-\frac{3}{8} R^{2}\right)$, which introduces a mass parameter $m$. The resulting theory, dubbed "new massive gravity" (NMG), proves to be unitary [8-10] (unlike its 4D predecessor [3]) and it propagates 
two massive states of both helicities \pm 2 in a Minkowski vacuum. ${ }^{1}$ Further generalisations of NMG are also possible. First of all, one may consider a hybrid parity-violating model which interpolates between TMG and NMG [6] and is known as "general massive gravity" (GMS). Its specific feature is that the \pm 2 helicity states have different masses $m_{ \pm}$. Furthermore, adding a cosmological term (in the spirit of cosmological TMG [13-16]) leads to cosmological GMG [6]. It turns out that all of these 3D models for massive gravity admit supersymmetric extensions.

Topologically massive supergravity (TMSG) with $\mathcal{N}=1$ was introduced in [17] and its cosmological extension followed in [13]. The off-shell versions of cosmological TMSG theories were presented in [18] for $\mathcal{N}=2$ and in [19] for $\mathcal{N}=3$ and $\mathcal{N}=4$. The off-shell $\mathcal{N}=1$ supergravity extensions of the models for massive gravity proposed in [6] were given in [20] (see also [21]), while the $\mathcal{N}=2$ case was studied in a recent paper [23].

The constructions in $[20,21]$ and [23] made use of component techniques. ${ }^{2}$ Such techniques are quite adept for deriving supergravity-matter systems with at most two derivatives. However, they can become rather involved when it comes to constructing higher-derivative couplings such as supersymmetric extensions of the curvature squared terms. ${ }^{3}$ For instance, the Ricci squared invariant in $\mathcal{N}=2$ supergravity with a chiral compensator was only given at the bosonic level in [23]. Moreover, the component formalism does not seem to provide a clear approach to higher derivative invariants with more than four derivatives. For this reason it is worth looking for alternative approaches.

There exist fully-fledged superspace formulations for off-shell 3D $\mathcal{N}$-extended conformal supergravity $[29,30]$, of which [29] is a gauged-fixed version of [30]. The $\mathrm{SO}(\mathcal{N})$ superspace approach of [29] has been used to construct general off-shell supergravity-matter couplings for $1 \leq \mathcal{N} \leq 4$. The conformal superspace of [30] has been applied to provide a universal construction of the conformal supergravity actions for $1 \leq \mathcal{N} \leq 6[31,32]$ (for each $\mathcal{N}$, the conformal supergravity action is a locally supersymmetric Lorentzian ChernSimons term required to formulate TMSG). Off-shell versions for 3D Poincaré and anti-de Sitter (AdS) supergravity theories naturally follow by coupling conformal supergravity to conformal compensators, see [33] for the complete description of the $\mathcal{N}=2$ case. In this paper we show that all the supergravity invariants required for the construction of the massive supergravity models proposed in $[20,21,23]$ naturally originate within the superspace approaches of $[29,30]$. In particular, the construction of four-derivative invariants in 3D $\mathcal{N}=2$ supergravity is analogous to that in $4 \mathrm{D} \mathcal{N}=1$ supergravity [34]. We also construct, for the first time, curvature squared invariants in $\mathcal{N}=3$ supergravity.

Before turning to the technical aspects of this work, we would like to make several comments concerning $\mathcal{N}=2$ supergravity. ${ }^{4}$ There are three off-shell formulations for 3D

\footnotetext{
${ }^{1}$ It has been claimed that NMG is power-counting renormalizable [11]. However, this statement is incorrect as shown in [12].

${ }^{2}$ It should be mentioned that the superspace formalism to derive all the $\mathcal{N}=1$ invariants given in $[20,21]$ has been available since 1979 [24-26]. However, the questions posed and answered in [20, 21] had not been asked by the authors of [24-26]. In principle, the off-shell formulation for $3 \mathrm{D} \mathcal{N}=1$ supergravity proposed in 1978 [27, 28] is also perfectly suitable for the explicit construction of the invariants given in [20, 21].

${ }^{3}$ The supersymmetric extensions of $R^{3}$ terms in $3 \mathrm{D} \mathcal{N}=1$ supergravity were constructed in [22].

${ }^{4}$ For early works on off-shell $3 \mathrm{D} \mathcal{N}=2$ supergravity, see [35-38]. In on-shell $3 \mathrm{D} \mathcal{N}=2$ supergravity, the matter couplings were studied in [39-41].
} 
$\mathcal{N}=2$ Poincaré and AdS supergravity theories [29, 33]: (i) type I minimal; (ii) type II minimal; and (iii) non-minimal. They differ by the structure of the conformal compensators employed. Type I minimal supergravity is a $3 \mathrm{D}$ analogue of the old minimal formulation for $4 \mathrm{D} \mathcal{N}=1$ supergravity [42-44] (see [26, 45, 46] for reviews). Type II minimal supergravity is a $3 \mathrm{D}$ analogue of the new minimal formulation ${ }^{5}$ for $4 \mathrm{D} \mathcal{N}=1$ supergravity [47] (see [26, 45] for reviews). The non-minimal supergravity theories are analogues of the following $4 \mathrm{D} \mathcal{N}=1$ theories: (i) non-minimal supergravity without a cosmological term [48-50]; and (ii) non-minimal AdS supergravity [51]. As shown by Achúcarro and Townsend [52], in three dimensions $\mathcal{N}$-extended AdS supergravity exists in several incarnations. They were called the $(p, q)$ AdS supergravity theories where the non-negative integers $p \geq q$ are such that $\mathcal{N}=p+q$. It was demonstrated in [52] that these theories are naturally associated with the $3 \mathrm{D}$ AdS supergroups $\operatorname{OSp}(\mathrm{p} \mid 2 ; \mathbb{R}) \times \operatorname{OSp}(\mathrm{q} \mid 2 ; \mathbb{R})$. There are two off-shell realisations for $(1,1)$ AdS supergravity [33], which are the type I theory with a cosmological term and the non-minimal AdS theory. There is only one off-shell realisation for $(2,0)$ AdS supergravity [33], which is the type II theory with a cosmological term. Strictly speaking, the terminology $(p, q)$ AdS supergravity should be used only for supergravity theories with a cosmological term. In the literature, however, the names $(1,1)$ and $(2,0)$ supergravity theories are also used for the type I and type II minimal formulations.

This paper is organised as follows. Section 2 is devoted to the description of $\mathcal{N}=1$ supergravity models. In sections 3,4 and $5, \mathcal{N}=2$ supergravity models with a chiral compensator, with a real linear compensator and a complex linear compensator are presented, respectively. In all the sections $2-5$ special attention is given to those models that describe massive supergravity. In section 6 we construct new invariants in $\mathcal{N}=3$ supergravity with a vector multiplet compensator. A discussion of our results and concluding comments are given in section 7 .

We have also included a few technical appendices. In appendix A we summarise the essential details of conformal superspace for $\mathcal{N} \leq 3$. Appendices $\mathrm{B}$ and $\mathrm{C}$ are devoted to prepotential deformations for $\mathcal{N}=1$ and $\mathcal{N}=2$ supergravity.

\section{$2 \mathcal{N}=1$ supergravity models}

The construction of $\mathcal{N}=1$ supergravity models in three dimensions can be performed using the conventional superspace formalism of [24-26]. It makes use of a curved superspace $\mathcal{M}^{3 \mid 2}$ parametrized by real bosonic $\left(x^{m}\right)$ and real fermionic $\left(\theta^{\mu}\right)$ coordinates $z^{M}=\left(x^{m}, \theta^{\mu}\right)$, where $m=0,1,2$ and $\mu=1,2$.

\subsection{Conventional superspace}

The superspace geometry is described in terms of covariant derivatives of the form

$$
\mathcal{D}_{A}=\left(\mathcal{D}_{a}, \mathcal{D}_{\alpha}\right)=E_{A}-\Omega_{A}
$$

\footnotetext{
${ }^{5}$ Unlike the new minimal formulation for $4 \mathrm{D} \mathcal{N}=1$ supergravity, the type II minimal formulation is suitable to describe AdS supergravity, which is a unique feature of three dimensions.
} 
Here the vector fields $E_{A}=E_{A}^{M} \partial / \partial z^{M}$ define the inverse vielbein, and

$$
\Omega_{A}=\frac{1}{2} \Omega_{A}^{b c} M_{b c}=-\Omega_{A}{ }^{b} M_{b}=\frac{1}{2} \Omega_{A}^{\beta \gamma} M_{\beta \gamma}
$$

is the Lorentz connection. The Lorentz generators with two vector indices $\left(M_{a b}=-M_{b a}\right)$, with one vector index $\left(M_{a}\right)$ and with two spinor indices $\left(M_{\alpha \beta}=M_{\beta \alpha}\right)$ are related to each other by the rules: $M_{a}=\frac{1}{2} \varepsilon_{a b c} M^{b c}$ and $M_{\alpha \beta}=\left(\gamma^{a}\right)_{\alpha \beta} M_{a}$. The Lorentz generators act on the covariant derivatives as follows:

$$
\left[M_{\alpha \beta}, \mathcal{D}_{\gamma}\right]=\varepsilon_{\gamma(\alpha} \mathcal{D}_{\beta)}, \quad\left[M_{a b}, \mathcal{D}_{c}\right]=2 \eta_{c[a} \mathcal{D}_{b]} .
$$

In the notation of [29], the covariant derivatives obey the following (anti-)commutation relations:

$$
\begin{aligned}
\left\{\mathcal{D}_{\alpha}, \mathcal{D}_{\beta}\right\}= & 2 \mathrm{i} \mathcal{D}_{\alpha \beta}-4 \mathrm{i} \mathcal{S} M_{\alpha \beta}, \\
{\left[\mathcal{D}_{\alpha \beta}, \mathcal{D}_{\gamma}\right]=} & -2 \varepsilon_{\gamma(\alpha} \mathcal{S} \mathcal{D}_{\beta)}+2 \varepsilon_{\gamma(\alpha} \mathcal{C}_{\beta) \delta \rho} M^{\delta \rho} \\
& +\frac{2}{3}\left(\mathcal{D}_{\gamma} \mathcal{S} M_{\alpha \beta}-4 \mathcal{D}_{(\alpha} \mathcal{S} M_{\beta) \gamma}\right), \\
{\left[\mathcal{D}_{a}, \mathcal{D}_{b}\right]=} & -\frac{\mathrm{i}}{2} \varepsilon_{a b c}\left(\gamma^{c}\right)^{\alpha \beta}\left\{\mathcal{C}_{\alpha \beta \gamma} \mathcal{D}^{\gamma}+\frac{4}{3} \mathcal{D}_{\alpha} \mathcal{S} \mathcal{D}_{\beta}-\mathcal{D}_{(\alpha} \mathcal{C}_{\beta \gamma \delta)} M^{\gamma \delta}\right. \\
& \left.+\frac{2}{3}\left(\mathcal{D}^{2}-6 \mathrm{i} \mathcal{S}\right) \mathcal{S} M_{\alpha \beta}\right\},
\end{aligned}
$$

where $\mathcal{S}$ and $\mathcal{C}_{\alpha \beta \gamma}$ are related to each other by the Bianchi identity

$$
\mathcal{D}^{\gamma} \mathcal{C}_{\alpha \beta \gamma}=-\frac{4 \mathrm{i}}{3} \mathcal{D}_{\alpha \beta} \mathcal{S}
$$

Practically all supergravity actions (with the action for conformal supergravity being a notable exception) may be realized as invariants of the form ${ }^{6}$

$$
S=\mathrm{i} \int \mathrm{d}^{3 \mid 2} z E \mathfrak{L}\left(\mathcal{T}, \mathcal{D} \mathcal{T}, \mathcal{D}^{2} \mathcal{T}, \cdots\right), \quad E^{-1}=\operatorname{Ber}\left(E_{A}{ }^{M}\right),
$$

where $\mathcal{T}$ schematically represents the torsion components appearing in the covariant derivative algebra (2.4). Various choices for $\mathfrak{L}$ lead to different supergravity models. As far as the higher-derivative supergravity invariants are concerned, the important observations are: (i) the top component of $\mathcal{S}$ gives a scalar curvature contribution; and (ii) a linear in $\theta$ component of $\mathcal{C}_{\alpha \beta \gamma}, \mathcal{D}_{(\alpha} \mathcal{C}_{\beta \gamma \delta)}$, contains the traceless part of the Ricci curvature tensor. Therefore, choosing $\mathfrak{L} \propto\left(\mathcal{D}^{\alpha} \mathcal{S}\right) \mathcal{D}_{\alpha} \mathcal{S}$ leads to a supersymmetric completion of the scalar curvature squared, while $\mathfrak{L} \propto \mathcal{C}^{\alpha \beta \gamma} \mathcal{C}_{\alpha \beta \gamma}$ produces the Ricci tensor squared along with some other contributions.

As is well known, gravity in $d>2$ dimensions can be realized as a Weyl invariant dynamical system describing conformal gravity coupled to a conformal compensator [53, 54]. It is also well known that similar formulations exist for various supergravity theories. Such

\footnotetext{
${ }^{6} \operatorname{In} \mathcal{N}$-extended superspace we use the notation $\mathrm{d}^{3 \mid 2 \mathcal{N}} z:=\mathrm{d}^{3} x \mathrm{~d}^{2 \mathcal{N}} \theta$. The $\mathcal{N}=1$ supergravity measure, $\mathrm{d}^{3 \mid 2} z E$, is imaginary.
} 
formulations are useful for certain applications, including the component reduction of supergravity models. It is especially suitable when the conformal supergravity action is a sector of the complete action of the theory under consideration. In three dimensions, $\mathcal{N}=1$ conformal supergravity can be described using the above curved superspace setting by requiring an additional gauge symmetry known as super-Weyl invariance. The algebra of covariant derivatives (2.4) is invariant under super-Weyl transformations [36, 55, 56] of the form ${ }^{7}$

$$
\begin{aligned}
& \mathcal{D}_{\alpha}^{\prime}=\mathrm{e}^{\frac{1}{2} \sigma}\left(\mathcal{D}_{\alpha}+\mathcal{D}^{\beta} \sigma M_{\alpha \beta}\right), \\
& \mathcal{D}_{a}^{\prime}=\mathrm{e}^{\sigma}\left(\mathcal{D}_{a}+\frac{\mathrm{i}}{2}\left(\gamma_{a}\right)^{\alpha \beta} \mathcal{D}_{\alpha} \sigma \mathcal{D}_{\beta}+\varepsilon_{a b c} \mathcal{D}^{b} \sigma M^{c}-\frac{\mathrm{i}}{8}\left(\gamma_{a}\right)^{\alpha \beta}\left(\mathcal{D}^{\gamma} \sigma\right) \mathcal{D}_{\gamma} \sigma M_{\alpha \beta}\right),
\end{aligned}
$$

with the parameter $\sigma$ being a real unconstrained superfield. The corresponding transformation of the torsion superfields is

$$
\mathcal{S}^{\prime}=\frac{\mathrm{i}}{2} \mathrm{e}^{\frac{3}{2} \sigma}\left(\mathcal{D}^{2}-2 \mathrm{i} \mathcal{S}\right) \mathrm{e}^{-\frac{1}{2} \sigma}, \quad \mathcal{C}_{\alpha \beta \gamma}^{\prime}=-\frac{1}{2} \mathrm{e}^{\frac{1}{2} \sigma}\left(\mathcal{D}_{(\alpha \beta} \mathcal{D}_{\gamma)}-2 \mathcal{C}_{\alpha \beta \gamma}\right) \mathrm{e}^{\sigma} .
$$

Every supergravity-matter action can be made super-Weyl invariant by coupling the fields to a conformal compensator $\varphi$, which is a nowhere vanishing scalar superfield with the super-Weyl transformation law

$$
\varphi^{\prime}=\mathrm{e}^{\frac{1}{2} \sigma} \varphi .
$$

Applying a finite super-Weyl transformation allows one to choose the gauge $\varphi=1$, in which the super-Weyl invariant action reduces to the original one.

The super-Weyl invariance (2.7) is intrinsic to conformal supergravity. The action for $\mathcal{N}=1$ conformal supergravity ${ }^{8}$ does not depend on $\varphi$ and is given by [58]

$$
\begin{aligned}
S_{\mathrm{CSG}}= & -2 \int \mathrm{d}^{3 \mid 2} z E \Omega^{\alpha \beta \gamma} \mathcal{G}_{\alpha \beta \gamma} \\
& +\frac{2}{3} \int \mathrm{d}^{3 \mid 2} z E\left\{\operatorname{tr}\left(\Omega^{\alpha} \Omega^{\beta} \Omega_{\alpha \beta}-2 \mathcal{S} \Omega^{\alpha} \Omega_{\alpha}\right)-\mathcal{S} \Omega^{\alpha \beta}{ }_{\alpha} \Omega^{\gamma}{ }_{\beta \gamma}\right\} \\
& +32 \mathrm{i} \int \mathrm{d}^{3 \mid 2} z E \mathcal{S}^{2},
\end{aligned}
$$

where we have used the matrix notation $\Omega_{A}=\left(\Omega_{A} \beta^{\gamma}\right)$ and introduced the tensor

$$
\mathcal{G}_{\alpha \beta \gamma}:=\mathcal{C}_{\alpha \beta \gamma}-\frac{4}{3} \varepsilon_{\alpha(\beta} \mathcal{D}_{\gamma)} \mathcal{S}, \quad \mathcal{D}^{\alpha} \mathcal{G}_{\alpha \beta \gamma}=0 .
$$

This tensor will be used for later considerations. Modulo an overall coefficient, the structures in the first and second lines of (2.9) are uniquely fixed by the condition of invariance under the local Lorentz transformations

$$
\delta_{K} \Omega_{A}^{b c}=K_{A}^{D} \Omega_{D}^{b c}-\mathcal{D}_{A} K^{b c} .
$$

The last term in (2.9) is uniquely fixed by requiring invariance under the super-Weyl transformations. Separate sectors of the superfield action (2.9) had appeared long ago [26, $36,55]$, but the complete action was given only in [58].

\footnotetext{
${ }^{7}$ Only infinitesimal super-Weyl transformations were given in $[36,55,56]$.

${ }^{8}$ The action for $\mathcal{N}=1$ conformal supergravity was originally constructed in components using the superconformal tensor calculus [57].
} 


\subsection{The superconformal setting}

Off-shell $\mathcal{N}$-extended conformal supergravity in three dimensions can be realized in superspace [30] as a gauge theory of the superconformal group $\operatorname{OSp}(\mathcal{N} \mid 4, \mathbb{R})$. This formulation, known as conformal superspace, is briefly reviewed in appendix A. It is the most powerful approach to derive off-shell conformal supergravity actions $[31,32]$. In the $\mathcal{N}=1$ case, the Weyl invariant formulation for conformal supergravity sketched above originates from conformal superspace by partially fixing certain local symmetries, see [30] for the details. Therefore, it is quite natural to carry out our subsequent analysis in conformal superspace; all results may be recast in the conventional superspace formalism by imposing the gauge conditions required. One of the advantages of conformal superspace is that it improves the complexity in performing component reduction.

Within the conformal superspace setting, the conformal compensator $\varphi$ has to be a primary superfield of dimension $1 / 2$,

$$
\mathbb{D} \varphi=\frac{1}{2} \varphi, \quad K_{A} \varphi=0 .
$$

We define the component fields of $\varphi$ as follows ${ }^{9}$

$$
l:=\varphi\left|, \quad \lambda_{\alpha}:=\mathrm{i} \nabla_{\alpha} \varphi\right|, \quad S:=\frac{\mathrm{i}}{2} \nabla^{2} \varphi \mid,
$$

where the bar-projection $[26,46,59]$ of a superfield $V(z)=V(x, \theta)$ is defined in the standard way $V|:=V(x, \theta)|_{\theta=0}$. Here we have introduced the operator

$$
\nabla^{2}:=\nabla^{\alpha} \nabla_{\alpha}
$$

Using $\varphi$ one can deform the covariant derivatives of conformal superspace $\nabla_{A}$ to new covariant derivatives $\mathscr{D}_{A}$ that are dimensionless and take primary superfields to primary ones. This procedure is very much like the one adopted in [51, 60-63] to construct Weyl invariant covariant derivatives. We define the new covariant derivatives as follows:

$$
\begin{aligned}
\mathscr{D}_{\alpha} & :=\frac{1}{\varphi}\left(\nabla_{\alpha}-2 \nabla^{\beta} \ln \varphi M_{\alpha \beta}-2 \nabla_{\alpha} \ln \varphi \mathbb{D}\right), \\
\mathscr{D}_{a} & :=\frac{\mathrm{i}}{4}\left(\gamma_{a}\right)^{\alpha \beta}\left\{\mathscr{D}_{\alpha}, \mathscr{D}_{\beta}\right\}-2 \mathscr{S} M_{a},
\end{aligned}
$$

where we have introduced the dimension zero primary superfield

$$
\mathscr{S}:=\frac{\mathrm{i}}{2 \varphi^{3}} \nabla^{2} \varphi .
$$

Note that $\varphi$ is covariantly constant with respect to $\mathscr{D}_{A}, \mathscr{D}_{A} \varphi=0$. When acting on a primary superfield, the covariant derivatives $\mathscr{D}_{A}$ satisfy the algebra

$$
\begin{aligned}
\left\{\mathscr{D}_{\alpha}, \mathscr{D}_{\beta}\right\}= & 2 \mathrm{i} \mathscr{D}_{\alpha \beta}-4 \mathrm{i} \mathscr{S} M_{\alpha \beta}, \\
{\left[\mathscr{D}_{\alpha \beta}, \mathscr{D}_{\gamma}\right]=} & -2 \varepsilon_{\gamma(\alpha} \mathscr{S} \mathscr{D}_{\beta)}+2 \varepsilon_{\gamma(\alpha} \mathscr{C}_{\beta) \delta \rho} M^{\delta \rho} \\
& +\frac{2}{3}\left(\mathscr{D}_{\gamma} \mathscr{S} M_{\alpha \beta}-4 \mathscr{D}_{(\alpha} \mathscr{S} M_{\beta) \gamma}\right),
\end{aligned}
$$

\footnotetext{
${ }^{9}$ The component fields of the conformal supergravity multiplet were elaborated in [31].
} 
where we have introduced

$$
\mathscr{C}_{\alpha \beta \gamma}:=-\frac{1}{2 \varphi} \nabla_{(\alpha \beta} \nabla_{\gamma)} \frac{1}{\varphi^{2}} .
$$

The algebra (2.17) formally coincides with (2.4). In fact, we can relate the superconformal framework presented above to the one of conventional superspace by gauge fixing the additional symmetries. The conformal boosts and $S$-supersymmetry transformations can be fixed by imposing the gauge condition

$$
B_{A}=0,
$$

which reduces conformal superspace to conventional superspace via the degauging procedure of [30]. The composites (2.16) and (2.18) become

$$
\begin{aligned}
\mathscr{S} & =\frac{\mathrm{i}}{2 \varphi^{3}}\left(\mathcal{D}^{2}-2 \mathrm{i} \mathcal{S}\right) \varphi, \\
\mathscr{C}_{\alpha \beta \gamma} & =-\frac{1}{2 \varphi}\left(\mathcal{D}_{(\alpha \beta} \mathcal{D}_{\gamma)}-2 \mathcal{C}_{\alpha \beta \gamma}\right) \frac{1}{\varphi^{2}} .
\end{aligned}
$$

One can then use the super-Weyl transformations to impose the gauge condition

$$
\varphi=1 \text {. }
$$

One can see that in the above gauge the composites (2.16) and (2.18) coincide with the torsion components $\mathcal{S}$ and $\mathcal{C}_{\alpha \beta \gamma}$ of conventional superspace.

We will make use of the composites (2.16) and (2.18) to construct supergravity invariants as superspace integrals. Superspace actions have the form

$$
S=\mathrm{i} \int \mathrm{d}^{3 \mid 2} z E \mathcal{L},
$$

where $\mathcal{L}=\overline{\mathcal{L}}$ is a real primary superfield of dimension 2 ,

$$
\mathbb{D} \mathcal{L}=2 \mathcal{L}, \quad K_{A} \mathcal{L}=0 .
$$

Using our constructions one may consider general actions of the form

$$
S=\mathrm{i} \int \mathrm{d}^{3 \mid 2} z E \varphi^{4} \mathfrak{L}\left(\mathscr{T}, \mathscr{D} \mathscr{T}, \mathscr{D}^{2} \mathscr{T}, \cdots\right),
$$

where $\mathfrak{L}$ is a dimensionless superfield constructed out of the torsion components $\mathscr{T}$ of the covariant derivatives $\mathscr{D}_{A}$. In particular, one can in principle construct general higher derivatives couplings. In this section, we will focus our attention on actions containing at most curvature squared terms.

In order to reduce the superspace actions to components we make use of the following component reduction formula [64]:

$$
\begin{aligned}
S & =\mathrm{i} \int \mathrm{d}^{3 \mid 2} z E \mathcal{L}, \\
& =-\frac{\mathrm{i}}{4} \int \mathrm{d}^{3} x e\left\{\nabla^{2}-\mathrm{i}\left(\gamma^{a}\right)^{\alpha \beta} \psi_{a \alpha} \nabla_{\beta}-\frac{1}{2} \varepsilon^{a b c}\left(\gamma_{a}\right)_{\alpha \beta} \psi_{b}{ }^{\alpha} \psi_{c}{ }^{\beta}\right\} \mathcal{L} \mid,
\end{aligned}
$$


where $e:=\operatorname{det}\left(e_{m}{ }^{a}\right)$. Here the component vielbein $e_{m}{ }^{a}$, its inverse $e_{a}{ }^{m}$ and the gravitino field $\psi_{a}{ }^{\beta}$ are defined by

$$
\begin{aligned}
& e_{m}{ }^{a}:=E_{m}{ }^{a} \mid, \quad e_{a}{ }^{m} e_{m}{ }^{b}=\delta_{a}^{b}, \quad e_{m}{ }^{a} e_{a}{ }^{n}=\delta_{m}^{n}, \\
& \psi_{a}{ }^{\beta}:=e_{a}{ }^{m} \psi_{m}{ }^{\beta}, \quad \psi_{m}{ }^{\alpha}:=2 E_{m}{ }^{\alpha} \mid .
\end{aligned}
$$

In what follows, we will mostly be interested in the bosonic sectors of locally supersymmetric actions, although by using the previous results it is straightforward to derive the full component actions.

Applying the component reduction formula, eq. (2.25), to our supergravity models, one will often find the appearance of terms such as $\nabla^{a} \nabla_{a} \varphi \mid$ and $\nabla^{a} \nabla_{a} \nabla^{b} \nabla_{b} \varphi \mid$. Such terms are of significance because they involve scalar curvature and Ricci curvature squared contributions. In general one finds for a primary scalar superfield $\phi$ of dimension $\Delta$ and lowest component $f:=\phi \mid$ the following results:

$$
\begin{aligned}
\nabla^{a} \nabla_{a} \phi \mid= & \left(D^{a} D_{a}+\frac{\Delta}{4} R\right) f+\text { fermion terms } \\
\nabla^{a} \nabla_{a} \nabla^{b} \nabla_{b} \phi \mid= & D^{a}\left(D_{a} D^{b} D_{b} f+\frac{\Delta}{4}\left(D_{a} R\right) f+\frac{\Delta}{4} R D_{a} f\right. \\
& \left.\quad+(2 \Delta-1)\left(R_{a b} D^{b} f-\frac{1}{4} R D_{a} f\right)\right) \\
& +(2 \Delta-1) R^{a b} D_{a} D_{b} f-\frac{(\Delta-3)}{4} R D^{a} D_{a} f \\
& +\Delta(2 \Delta-1) R^{a b} R_{a b} f-\frac{\Delta(9 \Delta-7)}{16} R^{2} f+\text { fermion terms. }
\end{aligned}
$$

Here we have introduced the covariant derivative

$$
D_{a}=e_{a}^{m}\left(\partial_{m}-\frac{1}{2} \omega_{m}^{b c} M_{b c}-b_{m} \mathbb{D}\right),
$$

where the Lorentz connection $\omega_{m}^{b c}$ and dilatation connection $b_{m}$ are defined as component projections of their corresponding superspace connections,

$$
\omega_{m}^{b c}=\Omega_{m}^{b c}\left|, \quad b_{m}=B_{m}\right| .
$$

The scalar curvature $R$ is constructed from the Lorentz curvature $R_{a b}{ }^{c d}$ as follows

$$
R=R_{a b}{ }^{a b},
$$

where the Lorentz curvature $R_{a b}{ }^{c d}$ is given by

$$
R_{a b}^{c d}=2 e_{a}^{m} e_{b}^{n}\left(\partial_{[m} \omega_{n]}^{a b}-2 \omega_{[m}^{c f} \omega_{n] f^{d}}\right) .
$$

In the cases we consider we will only need to make use of the results (2.27) in the gauge where $\phi=1$. For a more detailed discussion of the component results and conventions, the reader is referred to [31]. 
It should be mentioned that at the component level the gauge conditions (2.19) and (2.21) corresponds to setting

$$
l=1, \quad \lambda_{\alpha}=0, \quad b_{m}=0 .
$$

Here the first gauge condition fixes the dilatations, the second fixes the $S$-supersymmetry transformations and the last fixes the conformal boosts. We also point out that the top component $S$ of $\varphi$, eq. (2.13), does not vanish in the gauge $\varphi=1$. These gauge conditions are useful in deriving component actions corresponding to supergravity invariants.

\subsection{Supergravity invariants}

We now turn to describing locally supersymmetric invariants which contribute to massive supergravity actions.

\subsubsection{The supergravity action}

The standard $\mathcal{N}=1$ supergravity action with a cosmological term is given by

$$
S=\frac{1}{\kappa} S_{\mathrm{SG}}+\lambda S_{\mathrm{cos}}
$$

where

$$
S_{\mathrm{SG}}=8 \mathrm{i} \int \mathrm{d}^{3 \mid 2} z E \varphi^{4} \mathscr{S},
$$

is the supersymmetric Einstein-Hilbert action, and

$$
S_{\mathrm{cos}}=\mathrm{i} \int \mathrm{d}^{3 \mid 2} z E \varphi^{4}
$$

is the supersymmetric cosmological term. The functional $S_{\mathrm{SG}}$ gives rise to the EinsteinHilbert term $-\frac{1}{2} R$ once one reduces to components and imposes the gauge conditions (2.32). To see this one applies the component reduction formula (2.25) to the action (2.34). Keeping in mind the expression for $\mathscr{S}$ in terms of the compensator, eq. (2.16), one finds a term involving the component projection of $\nabla^{2} \nabla^{2} \varphi=-4 \nabla^{a} \nabla_{a} \varphi$. Finally, making use of (2.27) in the gauge $\varphi=1$ recovers the Einstein-Hilbert term. The full component action can be similarly computed. Here we are primarily concerned with the curvature dependence of our supergravity invariants.

\subsubsection{The $S^{n}$ invariants}

Keeping in mind the gauge condition (2.32), one can construct an invariant which contains an $S^{n}$ term, with $n$ a positive integer. Such a functional is given in terms of $\mathscr{S}$ as follows:

$$
S_{S^{n}}=\mathrm{i} \int \mathrm{d}^{3 \mid 2} z E \varphi^{4} \mathscr{S}^{n-1} .
$$

For $n=1$ and $n=2$ one recovers the supergravity cosmological term (2.35) and the Poincaré supergravity action (2.34), respectively. Similarly to the supergravity action one can check that the action (2.36) contains the contribution $-\frac{(n-1)}{16} R S^{n-2}$ for $n \geq 2$. The corresponding bosonic component action was given in [21]. For $n=1$ it coincides with the cosmological term, while for $n=2$ it gives the Einstein-Hilbert term. 


\subsubsection{The scalar curvature squared invariant}

A functional containing a scalar curvature term $R^{2}$ may be constructed using

$$
S_{\text {scalar }^{2}}=\int \mathrm{d}^{3 \mid 2} z E \varphi^{4}\left(\mathscr{D}^{\alpha} \mathscr{S}\right) \mathscr{D}_{\alpha} \mathscr{S} .
$$

Upon integration by parts the above functional may be taken to the equivalent form

$$
S_{\text {scalar }^{2}}=-\int \mathrm{d}^{3 \mid 2} z E \varphi \mathscr{S} \nabla^{2}(\varphi \mathscr{S})-2 S_{S^{4}} .
$$

One can check that the first term in $S_{\text {scalar }^{2}}$ gives rise to a scalar curvature squared term, $-\frac{1}{64} R^{2}$. At the component level a scalar curvature squared action was given in [20] using different techniques. Our curvature squared action (2.37) differs from the one in [20] by the addition of a multiple of the $S^{4}$ invariant.

\subsubsection{The Ricci curvature squared invariant}

An invariant containing a Ricci squared term, $R^{a b} R_{a b}$, is given by

$$
S_{\mathrm{Ricci}^{2}}=-2 \int \mathrm{d}^{3 \mid 2} z E \varphi^{4} \mathscr{C}^{\alpha \beta \gamma} \mathscr{C}_{\alpha \beta \gamma} .
$$

One can verify this readily by imposing the gauge conditions (2.19) and (2.21) and working in conventional superspace. It is not difficult to see that the component action will involve a Riemann curvature squared contribution, which leads to the Ricci squared term. In particular, in the gauge (2.32) one finds the curvature squared contribution to be

$$
R^{a b} R_{a b}-\frac{1}{3} R^{2}
$$

One can then show that the combination

$$
S_{\mathrm{Ricci}^{2}}^{(\text {pure }}:=S_{\mathrm{Ricci}^{2}}-\frac{64}{3} S_{\text {scalar }^{2}}
$$

gives a pure Ricci curvature squared invariant without any scalar curvature squared terms.

Remarkably one can write down an alternative invariant in a compact form that also gives rise to a Ricci squared term

$$
S_{\Sigma}=\int \mathrm{d}^{3 \mid 2} z E \frac{\Sigma}{\varphi}, \quad \Sigma:=\nabla^{a} \nabla_{a} \nabla^{2} \frac{1}{\varphi} .
$$

The dimension- $\frac{5}{2}$ superfield $\Sigma$ can be shown to be primary. Using eq. (2.27) one can check that in the gauge (2.32) the invariant (2.42) contains the following curvature squared contribution:

$$
R^{a b} R_{a b}-\frac{23}{64} R^{2}
$$

The relative coefficients between the Ricci squared and scalar curvature squared contributions exactly coincides with that of the Ricci squared invariant constructed at the bosonic level in [20]. The result thus appears to coincide with the Ricci squared invariant in [21] up to the addition of a multiple of the $S^{4}$ invariant. The form of the curvature squared 
terms tells us that the invariant (2.42) may be expressed in terms of a linear combination of the Ricci squared invariant $S_{\mathrm{Ricci}^{2}}$, the scalar curvature squared invariant $S_{\text {scalar }^{2}}$ and the $S^{4}$ invariant $S_{S^{4}}$.

There is another linear combination of $S_{\mathrm{Ricci}^{2}}$ and $S_{\mathrm{scalar}^{2}}$ that is worth mentioning. Here we make use of the gauges (2.19) and (2.21), and define the following invariant in conventional superspace

$$
S_{\mathrm{Ricci}^{2}}^{(\mathrm{YM})}:=S_{\mathrm{Ricci}^{2}}-\frac{16}{3} S_{\mathrm{scalar}^{2}} .
$$

The reason for the superscript (YM) will become clear shortly. The form of the action allows one to write it entirely in terms of the tensor $\mathcal{G}_{\alpha \beta \gamma}=\mathcal{G}_{\alpha(\beta \gamma)}$, eq. (2.10), which has the property $\mathcal{D}^{\alpha} \mathcal{G}_{\alpha \beta \gamma}=0$. The action reads

$$
S_{\mathrm{Ricci}^{2}}^{(\mathrm{YM})}=-2 \int \mathrm{d}^{3 \mid 2} z E \mathcal{G}^{\alpha \beta \gamma} \mathcal{G}_{\alpha \beta \gamma}=2 \int \mathrm{d}^{3 \mid 2} z E \operatorname{tr}\left\{\mathcal{G}^{\alpha} \mathcal{G}_{\alpha}\right\},
$$

where we have defined the Lorentz generator valued superfield $\mathcal{G}_{\alpha}:=\mathcal{G}_{\alpha}{ }^{\beta \gamma} M_{\beta \gamma}$. The form of the action makes clear a striking connection with the $\mathcal{N}=1$ super Yang-Mills action

$$
S_{\mathrm{YM}}=2 \int \mathrm{d}^{3 \mid 2} z E \operatorname{tr}\left\{\boldsymbol{G}^{\alpha} \boldsymbol{G}_{\alpha}\right\}
$$

with $\boldsymbol{G}_{\alpha}$ the field strength of a Yang-Mills multiplet which satisfies the divergenceless condition $\mathcal{D}^{\alpha} \boldsymbol{G}_{\alpha}=0$. Here $\mathcal{D}_{\alpha}$ is the Yang-Mills group gauge covariant derivative.

The fact that an invariant containing a Ricci squared term may be made to resemble the Yang-Mills action is most significant from a component perspective. In particular, in [6] the full component action for a supergravity invariant containing a Ricci squared term was efficiently constructed in the gauge (2.32) by reducing the problem to one of coupling a certain Yang-Mills multiplet to supergravity. ${ }^{10}$ The procedure is equivalent to judiciously replacing the Yang-Mills multiplet component fields with those of the component fields of $\mathcal{G}_{\alpha}{ }^{\beta \gamma}$, which transform as a Yang-Mills multiplet (with a Lorentz group index) by virtue of $\mathcal{D}^{\alpha} \mathcal{G}_{\alpha}{ }^{\beta \gamma}=0$. At the component level one can check that the invariant contains the curvature squared contribution $R^{a b} R_{a b}-\frac{1}{4} R^{2}$.

It is worth mentioning that although we imposed the gauge conditions (2.19) and (2.21) it is straightforward to restore the compensator. One simply uses the action

$$
S_{\mathrm{Ricci}^{2}}^{(\mathrm{YM})}=-2 \int \mathrm{d}^{3 \mid 2} z E \varphi^{4} \mathscr{G}^{\alpha \beta \gamma} \mathscr{G}_{\alpha \beta \gamma},
$$

where

$$
\mathscr{G}_{\alpha}{ }^{\beta \gamma}=\mathscr{C}_{\alpha}{ }^{\beta \gamma}+\frac{4}{3} \delta_{\alpha}^{(\beta} \mathscr{D}^{\gamma)} \mathscr{S} .
$$

Although we have restricted our attention here to curvature squared invariants, our approach makes it possible to generate locally supersymmetric functionals containing higher powers of the curvature tensor and its covariant derivatives. All such invariants are described by actions of the form (2.24) where $\mathscr{T}$ denotes the primary dimensionless superfields (2.20). Among the descendants of (2.20), the following rank 3 symmetric spinor

$$
\mathscr{W}_{\alpha \beta \gamma}=-\mathrm{i} \mathscr{D}^{2} \mathscr{C}_{\alpha \beta \gamma}-2 \mathscr{D}_{(\alpha \beta} \mathscr{D}_{\gamma)} \mathscr{S}-8 \mathscr{S} \mathscr{C}_{\alpha \beta \gamma}
$$

\footnotetext{
${ }^{10}$ This is often referred to as the "Yang-Mills trick."
} 
plays a special role. It is related to the super-Cotton tensor [58], $W_{\alpha \beta \gamma}$, by the rule

$$
W_{\alpha \beta \gamma}=\varphi^{5} \mathscr{W}_{\alpha \beta \gamma} .
$$

The super-Cotton tensor has been written in terms of $\varphi$ to make clear how it is related to the torsion components in conventional superspace. It should be kept in mind that it is actually independent of the compensator $\varphi$. Given a curved superspace background, it is conformally flat if and only if $W_{\alpha \beta \gamma}$ is equal to zero [30]. This property explains the geometric meaning of the super-Cotton tensor. The super-Cotton tensor contains the ordinary Cotton tensor as the component field $\nabla_{(\alpha} W_{\beta \gamma \delta)} \mid$ and obeys the equation [30]

$$
\nabla^{\gamma} W_{\alpha \beta \gamma}=0
$$

\subsection{Models for massive supergravity}

Using the invariants constructed in the previous section one can build models for massive supergravity. The actions for these models are built out of a linear combination of the supergravity invariants together with the action for conformal supergravity. In this section we analyse the dynamical properties of such theories and derive the necessary conditions for massive supergravity.

We begin by considering a general $\mathcal{N}=1$ supergravity model described by the action

$$
S=\lambda S_{\mathrm{cos}}+\frac{1}{\kappa} S_{\mathrm{SG}}+\mu_{0} S_{\mathcal{S}^{3}}+\mu_{1} S_{\mathcal{S}^{4}}+\mu_{2} S_{\mathrm{scalar}^{2}}+\mu_{3} S_{\mathrm{Ricci}^{2}}+\frac{1}{\mu} S_{\mathrm{CSG}},
$$

where $S_{\mathrm{CSG}}$ denotes the $\mathcal{N}=1$ conformal supergravity action, eq. (2.9). In what follows we will assume that $\mu_{3} \geq 0$ as in $[20,21]$.

It is an instructive exercise to derive the equations of motion in the theory with action (2.52). Varying the action (2.52) with respect to the compensator $\varphi$ leads to the equation

$$
\begin{aligned}
0= & \lambda+\frac{4}{\kappa} \mathscr{S}-\mathrm{i} \mu_{3} \mathscr{C}^{\alpha \beta \gamma} \mathscr{C}_{\alpha \beta \gamma}-\frac{\mu_{1}}{2} \mathscr{S}^{3} \\
& +\frac{\mathrm{i}}{4} \mu_{0} \mathscr{D}^{2} \mathscr{S}+\mathrm{i}\left(\frac{3}{8} \mu_{1}-\frac{3}{2} \mu_{2}\right) \mathscr{D}^{2} \mathscr{S}^{2}+\frac{\mathrm{i} \mu_{2}}{2}\left(\mathscr{D}^{\alpha} \mathscr{S}\right) \mathscr{D}_{\alpha} \mathscr{S} \\
& +\left(\mu_{2}-\frac{8}{3} \mu_{3}\right) \mathscr{D}^{a} \mathscr{D}_{a} \mathscr{S} .
\end{aligned}
$$

There is no contribution proportional to $1 / \mu$ since the conformal supergravity action is independent of the compensator. The equation of motion for the conformal supergravity prepotential is

$$
\frac{1}{\mu} W_{\alpha \beta \gamma}+T_{\alpha \beta \gamma}=0
$$

where

$$
\varphi^{-5} T_{\alpha \beta \gamma}=\frac{1}{\kappa} \mathbb{T}_{\alpha \beta \gamma}^{(\mathrm{SG})}+\mu_{0} \mathbb{T}_{\alpha \beta \gamma}^{\left(S^{3}\right)}+\mu_{1} \mathbb{T}^{\left(S^{4}\right)}{ }_{\alpha \beta \gamma}+\mu_{2} \mathbb{T}^{\left(\mathrm{sca}^{2}\right)}{ }_{\alpha \beta \gamma}+\mu_{3} \mathbb{T}^{\left(\mathrm{Ric}^{2}\right)}{ }_{\alpha \beta \gamma}
$$


with the right-hand side consisting of the following contributions:

$$
\begin{aligned}
\mathbb{T}^{(\mathrm{SG})}{ }_{\alpha \beta \gamma}= & -2 \mathscr{C}_{\alpha \beta \gamma}, \\
\mathbb{T}^{\left(S^{n}\right)}{ }_{\alpha \beta \gamma}= & \frac{n}{16}\left(\mathscr{D}_{(\alpha \beta} \mathscr{D}_{\gamma)}+2 \mathscr{C}_{\alpha \beta \gamma}\right) \mathscr{S}^{n-1}, \\
\mathbb{T}^{\left(\mathrm{sca}^{2}{ }_{\alpha \beta \gamma}=\right.} & 2\left(\mathscr{D}_{(\alpha} \mathscr{S}\right) \mathscr{D}_{\beta \gamma)} \mathscr{S}-\frac{\mathrm{i}}{4}\left(\mathscr{D}_{(\alpha \beta} \mathscr{D}_{\gamma)}+2 \mathscr{C}_{\alpha \beta \gamma}\right) \mathscr{D}^{2} \mathscr{S}, \\
\mathbb{T}^{\left(\mathrm{Ric}^{2}{ }_{\alpha \beta \gamma}=\right.} & -4 \mathscr{D}^{a} \mathscr{D}_{a} \mathscr{C}_{\alpha \beta \gamma}+\frac{8 \mathrm{i}}{3} \mathscr{D}_{(\alpha \beta} \mathscr{D}_{\gamma)} \mathscr{D}^{2} \mathscr{S}-4 \mathrm{i} \mathscr{C}_{\alpha \beta \gamma} \mathscr{D}^{2} \mathscr{S}+4 \mathrm{i} \mathscr{S} \mathscr{D}^{2} \mathscr{C}_{\alpha \beta \gamma} \\
& +32 \mathscr{S}_{(\alpha \beta} \mathscr{D}_{\gamma)} \mathscr{S}+12 \mathscr{C}_{(\alpha \beta}{ }^{\rho} \mathscr{D}_{\gamma) \rho} \mathscr{S}+\frac{56}{3}\left(\mathscr{D}_{(\alpha \beta} \mathscr{S}\right) \mathscr{D}_{\gamma)} \mathscr{S} \\
& +\frac{8}{3} \mathscr{K}_{\alpha \beta \gamma \delta} \mathscr{D}^{\delta} \mathscr{S}-16 \mathscr{C}_{(\alpha}{ }^{\delta \rho} \mathscr{K}_{\beta \gamma) \delta \rho}+12 \mathscr{S}^{2} \mathscr{C}_{\alpha \beta \gamma} .
\end{aligned}
$$

Here we have denoted

$$
\mathscr{K}_{\alpha \beta \gamma \delta}=\mathrm{i} \mathscr{D}_{(\alpha} \mathscr{C}_{\beta \gamma \delta)} .
$$

The equation of motion (2.54) can be obtained by using the results in appendix B, which imply

$$
\delta S_{\mathrm{CSG}}=\mathrm{i} \int \mathrm{d}^{3 \mid 2} z E \delta \Psi^{\alpha \beta \gamma} W_{\alpha \beta \gamma}, \quad \delta\left(S-\frac{1}{\mu} S_{\mathrm{CSG}}\right)=\mathrm{i} \int \mathrm{d}^{3 \mid 2} z E \delta \Psi^{\alpha \beta \gamma} T_{\alpha \beta \gamma} .
$$

It is seen that (2.54) does not involve the cosmological constant $\lambda$. This is due to the fact that the cosmological term (2.35) does not depend on the conformal supergravity prepotential. It also follows from the analysis in appendix $\mathrm{B}$ that $T_{\alpha \beta \gamma}$ obeys the conservation equation

$$
\nabla^{\gamma} T_{\alpha \beta \gamma}=0
$$

provided the compensator is subject to its equation of motion (2.53).

By construction, the supergravity equations (2.53) and (2.54) are super-Weyl invariant. Upon imposing the gauge (2.19) and reducing to conventional superspace, the local superWeyl symmetry may be fixed by imposing the gauge

$$
\varphi=1
$$

which amounts to replacing $\mathscr{D}_{A} \rightarrow \mathcal{D}_{A}, \mathscr{C}_{\alpha \beta \gamma} \rightarrow \mathcal{C}_{\alpha \beta \gamma}$ and $\mathscr{S} \rightarrow \mathcal{S}$ everywhere. The superWeyl invariance can always be restored by performing an inverse replacement. The gauge condition (2.58) will be assumed in what follows.

The supergravity equations of motion have maximally supersymmetric solutions. Maximally supersymmetric backgrounds are specified by the conditions

$$
\mathcal{C}_{\alpha \beta \gamma}=0, \quad \mathcal{S}=\text { const },
$$

which imply that the algebra of covariant derivatives (2.4) drastically simplifies

$$
\begin{aligned}
\left\{\mathcal{D}_{\alpha}, \mathcal{D}_{\beta}\right\} & =2 \mathrm{i} \mathcal{D}_{\alpha \beta}-4 \mathrm{i} \mathcal{S} M_{\alpha \beta}, \\
{\left[\mathcal{D}_{\alpha \beta}, \mathcal{D}_{\gamma}\right] } & =-2 \varepsilon_{\gamma(\alpha} \mathcal{S D}_{\beta)}, \\
{\left[\mathcal{D}_{a}, \mathcal{D}_{b}\right] } & =-4 \mathcal{S}^{2} M_{a b} .
\end{aligned}
$$

Such a superspace describes either anti-de Sitter geometry for $\mathcal{S} \neq 0$ or a flat spacetime for $\mathcal{S}=0$. 
Let us look for a maximally supersymmetric background (2.59) with $\mathcal{S}=\mathcal{S}_{0}$ which is a solution of the supergravity equations of motion. In this case we have

$$
W_{\alpha \beta \gamma}=T_{\alpha \beta \gamma}=0,
$$

and therefore the equation $(2.54 \mathrm{a})$ is satisfied identically, while the equation on the compensator, eq. (2.53), becomes algebraic

$$
0=\lambda+\frac{4}{\kappa} \mathcal{S}_{0}-\frac{\mu_{1}}{2} \mathcal{S}_{0}^{3} .
$$

This cubic equation in $\mathcal{S}_{0}$ coincides with the one found in [20,21]. The real solutions of this equation (at least one real solution always exists) determine the maximally supersymmetric solutions of the supergravity theory under consideration.

One may impose the constraint

$$
\mu_{2}=\frac{8}{3} \mu_{3},
$$

which reduces the dynamical system (2.52) to a six-parameter subclass of models. In general this leads to a propagating scalar mode, which is eliminated in the case of generalized massive supergravity (GMSG) via a further choice of coefficients. At the component level one finds the equation of motion on the compensator to be

$$
\begin{aligned}
\lambda+\frac{4}{\kappa} S & -\frac{3 \mu_{0}}{2} S^{2}+\left(12 \mu_{2}-5 \mu_{1}\right) S^{3} \\
& =-\frac{1}{16}\left(\mu_{0}+\left(3 \mu_{1}-12 \mu_{2}\right) S\right) R+\text { fermion terms }
\end{aligned}
$$

where we have used

$$
\mathcal{D}^{2} \mathcal{S} \mid=6 \mathrm{i} S^{2}-\frac{\mathrm{i}}{4} R+\text { fermion terms }
$$

One can solve eq. (2.64) for $S$ in terms of the scalar curvature $R$. At the bosonic level this was worked out in [21] and leads to an action non-polynomial in $R$. It is worth mentioning that although we have suppressed the fermionic terms for simplicity, it is a straightforward exercise to recover them from eq. (2.53).

One may consider perturbations in the supergravity model (2.63) from the maximally supersymmetric solution:

$$
\mathcal{S}=\mathcal{S}_{0}+\Delta \mathcal{S}, \quad \mathcal{C}_{\alpha \beta \gamma}=\Delta \mathcal{C}_{\alpha \beta \gamma}
$$

The equation of motion on the compensator becomes

$$
0=\mathrm{i}\left[\frac{\mu_{0}}{4}+\left(\frac{3}{4} \mu_{1}-3 \mu_{2}\right) \mathcal{S}_{0}\right] \mathcal{D}^{2} \Delta \mathcal{S}+\left[\frac{4}{\kappa}-\frac{3 \mu_{1}}{2} \mathcal{S}_{0}^{2}\right] \Delta \mathcal{S}
$$

When the coefficient for $\mathcal{D}^{2} \Delta \mathcal{S}$ in eq. (2.67) does not vanish,

$$
\frac{\mu_{0}}{4}+\left(\frac{3}{4} \mu_{1}-3 \mu_{2}\right) \mathcal{S}_{0} \neq 0
$$


we have the equation

$$
\left(\frac{\mathrm{i}}{2} \mathcal{D}^{2}-m\right) \Delta \mathcal{S}=0 \quad \Longrightarrow\left(\mathcal{D}^{a} \mathcal{D}_{a}-m^{2}\right) \Delta \mathcal{S}=0
$$

where $m$ is given by

$$
m=-\frac{8-3 \kappa \mu_{1} \mathcal{S}_{0}^{2}}{\kappa\left(\mu_{0}+\left(3 \mu_{1}-12 \mu_{2}\right) \mathcal{S}_{0}\right)} .
$$

Hence in this case $\Delta \mathcal{S}$ becomes propagating.

Instead of (2.68) we may impose the condition

$$
\mu_{0}+\left(3 \mu_{1}-12 \mu_{2}\right) \mathcal{S}_{0}=0
$$

and also assume that

$$
\frac{4}{\kappa}-\frac{3 \mu_{1}}{2} \mathcal{S}_{0}^{2} \neq 0
$$

Then eq. (2.67) gives

$$
\Delta \mathcal{S}=0 .
$$

Linearising the Bianchi identity (2.5) about the background chosen and taking into account $\Delta \mathcal{S}=0$, we obtain the divergenceless condition

$$
\mathcal{D}^{\gamma} \Delta \mathcal{C}_{\alpha \beta \gamma}=0 \quad \Longrightarrow \quad \mathcal{D}^{2} \Delta \mathcal{C}_{\alpha \beta \gamma}=2 \mathrm{i} \mathcal{D}_{\alpha}^{\delta} \Delta \mathcal{C}_{\beta \gamma \delta}+10 \mathrm{i} \mathcal{S}_{0} \Delta \mathcal{C}_{\alpha \beta \gamma}
$$

At this point it is worth remarking on a property of symmetric divergenceless spinors in the AdS background (2.60) chosen. In general, given a symmetric rank- $n$ spinor, $T_{\alpha_{1} \cdots \alpha_{n}}=$ $T_{\left(\alpha_{1} \cdots \alpha_{n}\right)}$, it holds that

$$
\mathcal{D}^{\beta} T_{\alpha_{1} \cdots \alpha_{n-1} \beta}=0 \quad \Longrightarrow \quad \mathcal{D}^{\beta} \mathcal{D}^{2} T_{\alpha_{1} \cdots \alpha_{n-1} \beta}=0 .
$$

The linearised torsion $\Delta \mathcal{C}_{\alpha \beta \gamma}$ is an example of such a superfield. In particular, the operator $\frac{i}{2} \mathcal{D}^{2}$ preserves the divergenceless condition of $\Delta \mathcal{C}_{\alpha \beta \gamma}$.

Using eq. (2.74), the supergravity equation of motion can be written in terms of vector covariant derivatives as follows

$$
\begin{aligned}
\mu_{3} \mathcal{D}^{a} \mathcal{D}_{a} \Delta \mathcal{C}_{\alpha \beta \gamma} & +\left(2 \mu_{3} \mathcal{S}_{0}-\frac{1}{2 \mu}\right) \mathcal{D}_{\alpha}{ }^{\delta} \Delta \mathcal{C}_{\beta \gamma \delta} \\
& +\left[\frac{1}{2 \kappa}-\frac{1}{2 \mu} \mathcal{S}_{0}-\left(\frac{3}{32} \mu_{0}-7 \mu_{3}\right) \mathcal{S}_{0}^{2}-\frac{1}{8} \mu_{1} \mathcal{S}_{0}^{3}\right] \Delta \mathcal{C}_{\alpha \beta \gamma}=0 .
\end{aligned}
$$

When $\mu_{3} \neq 0$ the equation of motion may be written in the following factorized form

$$
\left(\frac{\mathrm{i}}{2} \mathcal{D}^{2}+m_{-}\right)\left(\frac{\mathrm{i}}{2} \mathcal{D}^{2}-m_{+}\right) \Delta \mathcal{C}_{\alpha \beta \gamma}=0,
$$

where the constants $m_{+}$and $m_{-}$are such that

$$
\begin{aligned}
m_{+} m_{-} & =-\frac{1}{2 \mu_{3}}\left[\frac{1}{\kappa}+\frac{4}{\mu} \mathcal{S}_{0}-3\left(\frac{1}{16} \mu_{0}-8 \mu_{3}\right) \mathcal{S}_{0}^{2}-\frac{\mu_{1}}{4} \mathcal{S}_{0}^{3}\right], \\
m_{+}-m_{-} & =8 \mathcal{S}_{0}+\frac{1}{2 \mu_{3} \mu} .
\end{aligned}
$$


The constants $m_{+}$and $m_{-}$are real when the following inequality is satified:

$$
\frac{1}{\kappa}-\frac{1}{8 \mu_{3} \mu^{2}}-\left(\frac{3}{16} \mu_{0}+8 \mu_{3}\right) \mathcal{S}_{0}^{2}-\frac{\mu_{1}}{4} \mathcal{S}_{0}^{3} \leq 0
$$

The supergravity equations of motion have massive solutions in a number of cases. For instance, generalized massive supergravity $[20,21]$ is characterised by a negative EinsteinHilbert term, $\kappa<0$, while the case of new topologically massive supergravity $[20,21]$ is characterized by $\kappa \rightarrow \infty$. Furthermore, new massive supergravity occurs in the case $\mu_{3} \neq 0$ and $\mu \rightarrow \infty$. In this case it is straightforward to verify that about a Minkowski background $\left(\mathcal{S}_{0}=0\right)$ we have the massive equation

$$
\left(\partial^{a} \partial_{a}-\tilde{m}^{2}\right) \Delta \mathcal{C}_{\alpha \beta \gamma}=0, \quad \tilde{m}^{2}:=m_{+} m_{-}=-\frac{1}{2 \mu_{3} \kappa},
$$

where $\tilde{m}$ is real for a negative Einstein-Hilbert term, $\kappa<0$.

In the case when $\mu_{3}=0$ and $\mu$ is finite we have the equation

$$
\left(\frac{\mathrm{i}}{2} \mathcal{D}^{2}-\hat{m}\right) \Delta \mathcal{C}_{\alpha \beta \gamma}=0
$$

where $\hat{m}$ is given by

$$
\hat{m}=-\left(\frac{\mu}{\kappa}+4 \mathcal{S}_{0}-\frac{3 \mu_{0} \mu}{16} \mathcal{S}_{0}^{3}-\frac{\mu \mu_{1}}{4} \mathcal{S}_{0}^{3}\right) .
$$

About a Minkowski background it is straightforward to verify that

$$
\left(\partial^{a} \partial_{a}-\hat{m}^{2}\right) \Delta \mathcal{C}_{\alpha \beta \gamma}=0 .
$$

Note that when $\mu_{0}=\mu_{1}=0$ the above coincides with topologically massive supergravity.

We have reduced the supergravity models to those considered in [20, 21]. The analysis of unitarity for such theories may be carried out as in [20,21].

\section{$3 \mathcal{N}=2$ supergravity models with a chiral compensator}

All known off-shell formulations for $3 \mathrm{D} \mathcal{N}=2$ supergravity $[29,33]$ can be formulated in conventional superspace with structure group $\mathrm{SL}(2, \mathbb{R}) \times \mathrm{U}(1)_{R}$. This curved superspace $\mathcal{M}^{3 \mid 4}$ is parametrised by local bosonic $\left(x^{m}\right)$ and fermionic $\left(\theta^{\mu}, \bar{\theta}_{\mu}\right)$ coordinates $z^{M}=$ $\left(x^{m}, \theta^{\mu}, \bar{\theta}_{\mu}\right)$, where the Grassmann variables $\theta^{\mu}$ and $\bar{\theta}_{\mu}$ are related to each other by complex conjugation: $\overline{\theta^{\mu}}=\bar{\theta}^{\mu}$.

\subsection{Conventional superspace}

The covariant derivatives of conventional $\mathcal{N}=2$ superspace $\mathcal{D}_{A}=\left(\mathcal{D}_{a}, \mathcal{D}_{\alpha}, \overline{\mathcal{D}}^{\alpha}\right)$ have the form

$$
\mathcal{D}_{A}=E_{A}-\Omega_{A}-\mathrm{i} \Phi_{A} J,
$$

with $J$ the $R$-symmetry generator acting on the covariant derivatives as follows:

$$
\left[J, \mathcal{D}_{\alpha}\right]=\mathcal{D}_{\alpha}, \quad\left[J, \overline{\mathcal{D}}^{\alpha}\right]=-\overline{\mathcal{D}}^{\alpha}, \quad\left[J, \mathcal{D}_{a}\right]=0 .
$$


In order to describe $\mathcal{N}=2$ conformal supergravity, the torsion has to obey the covariant constraints proposed in [38]. The resulting algebra of covariant derivatives is $[29,33]$

$$
\begin{aligned}
\left\{\mathcal{D}_{\alpha}, \mathcal{D}_{\beta}\right\}= & -4 \overline{\mathcal{R}} M_{\alpha \beta} \\
\left\{\mathcal{D}_{\alpha}, \overline{\mathcal{D}}_{\beta}\right\}= & -2 \mathrm{i}\left(\gamma^{c}\right)_{\alpha \beta} \mathcal{D}_{c}-2 \mathcal{C}_{\alpha \beta} J-4 \mathrm{i} \varepsilon_{\alpha \beta} \mathcal{S} J+4 \mathrm{i} \mathcal{S} M_{\alpha \beta}-2 \varepsilon_{\alpha \beta} \mathcal{C}^{\gamma \delta} M_{\gamma \delta} \\
{\left[\mathcal{D}_{a}, \mathcal{D}_{\beta}\right]=} & \mathrm{i} \varepsilon_{a b c}\left(\gamma^{b}\right)_{\beta}{ }^{\gamma} \mathcal{C}^{c} \mathcal{D}_{\gamma}+\left(\gamma_{a}\right)_{\beta}{ }^{\gamma} \mathcal{S} \mathcal{D}_{\gamma}-\mathrm{i}\left(\gamma_{a}\right)_{\beta \gamma} \overline{\mathcal{R}} \overline{\mathcal{D}}^{\gamma}+\mathrm{i}\left(\gamma_{a}\right)_{\beta}{ }^{\gamma} \mathcal{D}_{(\gamma} \mathcal{C}_{\delta \rho)} M^{\delta \rho} \\
& -\frac{1}{3}\left(2 \mathcal{D}_{\beta} \mathcal{S}+\mathrm{i} \overline{\mathcal{D}}_{\beta} \overline{\mathcal{R}}\right) M_{a}-\frac{2}{3} \varepsilon_{a b c}\left(\gamma^{b}\right)_{\beta}{ }^{\alpha}\left(2 \mathcal{D}_{\alpha} \mathcal{S}+\mathrm{i} \overline{\mathcal{D}}_{\alpha} \overline{\mathcal{R}}\right) M^{c} \\
& +\frac{\mathrm{i}}{2}\left(\left(\gamma_{a}\right)^{\alpha \gamma} \mathcal{D}_{(\alpha} \mathcal{C}_{\beta \gamma)}+\frac{1}{3}\left(\gamma_{a}\right)_{\beta}{ }^{\gamma}\left(8 \mathrm{i} \mathcal{D}_{\gamma} \mathcal{S}-\overline{\mathcal{D}}_{\gamma} \overline{\mathcal{R}}\right)\right) J
\end{aligned}
$$

where the $\mathrm{U}(1)_{R}$ charges of the torsion superfields $\mathcal{R}, \overline{\mathcal{R}}$ and $\mathcal{C}_{\alpha \beta}$ are $-2,+2$ and 0 , respectively. They also satisfy the Bianchi identities

$$
\mathcal{D}_{\alpha} \overline{\mathcal{R}}=0, \quad \mathcal{D}^{\beta} \mathcal{C}_{\alpha \beta}=-\frac{1}{2}\left(\overline{\mathcal{D}}_{\alpha} \overline{\mathcal{R}}+4 \mathrm{i} \mathcal{D}_{\alpha} \mathcal{S}\right)
$$

The algebra of covariant derivatives given by (3.3) does not change under the superWeyl transformation $[29,33]$

$$
\begin{aligned}
\mathcal{D}^{\prime}{ }_{\alpha}= & \mathrm{e}^{\frac{1}{2} \sigma}\left(\mathcal{D}_{\alpha}+\mathcal{D}^{\gamma} \sigma M_{\gamma \alpha}-\mathcal{D}_{\alpha} \sigma J\right) \\
\mathcal{D}^{\prime}{ }_{a}= & \mathrm{e}^{\sigma}\left(\mathcal{D}_{a}-\frac{\mathrm{i}}{2}\left(\gamma_{a}\right)^{\gamma \delta} \mathcal{D}_{\gamma} \sigma \overline{\mathcal{D}}_{\delta}-\frac{\mathrm{i}}{2}\left(\gamma_{a}\right)^{\gamma \delta} \overline{\mathcal{D}}_{\gamma} \sigma \mathcal{D}_{\delta}+\varepsilon_{a b c} \mathcal{D}^{b} \sigma M^{c}\right. \\
& \left.-\frac{\mathrm{i}}{2}\left(\mathcal{D}^{\gamma} \sigma\right) \overline{\mathcal{D}}_{\gamma} \sigma M_{a}-\frac{\mathrm{i}}{24}\left(\gamma_{a}\right)^{\gamma \delta} \mathrm{e}^{-3 \sigma}\left[\mathcal{D}_{\gamma}, \overline{\mathcal{D}}_{\delta}\right] \mathrm{e}^{3 \sigma} J\right)
\end{aligned}
$$

which induces the following transformation of the torsion tensors:

$$
\begin{aligned}
\mathcal{S}^{\prime} & =\mathrm{e}^{\sigma}\left(\mathcal{S}+\frac{\mathrm{i}}{4} \mathcal{D}^{\gamma} \overline{\mathcal{D}}_{\gamma} \sigma\right), \\
\mathcal{C}_{a}^{\prime} & =\left(\mathcal{C}_{a}+\frac{1}{8}\left(\gamma_{a}\right)^{\gamma \delta}\left[\mathcal{D}_{\gamma}, \overline{\mathcal{D}}_{\delta}\right]\right) \mathrm{e}^{\sigma}, \\
\mathcal{R}^{\prime} & =-\frac{1}{4} \mathrm{e}^{2 \sigma}\left(\overline{\mathcal{D}}^{2}-4 \mathcal{R}\right) \mathrm{e}^{-\sigma} .
\end{aligned}
$$

Here the parameter $\sigma$ is an arbitrary real scalar superfield and we have defined $\mathcal{D}^{2}:=\mathcal{D}^{\alpha} \mathcal{D}_{\alpha}$ and $\overline{\mathcal{D}}^{2}:=\overline{\mathcal{D}}_{\alpha} \overline{\mathcal{D}}^{\alpha}$. The super-Weyl invariance (3.5) is intrinsic to conformal supergravity. For every supergravity-matter system, its action is required to be a super-Weyl invariant functional of the supergravity Weyl multiplet coupled to certain conformal compensators, see $[29,33]$ for more details.

There exists an important super-Weyl invariant descendent of the torsion components $\mathcal{C}_{\alpha \beta}$ and $\mathcal{S}$ that is worth mentioning. Using the above super-Weyl transformation laws, one can check that the following real vector superfield [68]

$$
\mathcal{W}_{\alpha \beta}=-\frac{\mathrm{i}}{4}\left[\mathcal{D}^{\gamma}, \overline{\mathcal{D}}_{\gamma}\right] \mathcal{C}_{\alpha \beta}+\frac{1}{2}\left[\mathcal{D}_{(\alpha}, \overline{\mathcal{D}}_{\beta)}\right] \mathcal{S}+2 \mathcal{S C}_{\alpha \beta}
$$

transforms homogeneously,

$$
\mathcal{W}_{\alpha \beta}^{\prime}=\mathrm{e}^{2 \sigma} \mathcal{W}_{\alpha \beta}
$$


The superfield is the $\mathcal{N}=2$ super-Cotton tensor and it vanishes if and only if the curved superspace is conformally flat [30]. Using the Bianchi identities one can find the following equivalent form for the super-Cotton tensor [18]:

$$
\mathcal{W}_{a}=-\frac{1}{2}\left(\gamma_{a}\right)^{\alpha \beta} \mathcal{W}_{\alpha \beta}=\frac{1}{4}\left(\gamma_{a}\right)^{\alpha \beta}\left[\mathcal{D}_{(\alpha}, \overline{\mathcal{D}}_{\beta)}\right] \mathcal{S}-\varepsilon_{a b c} \mathcal{D}^{b} \mathcal{C}^{c}-2 \mathcal{S C}_{a}
$$

The covariant derivative algebra of conformal superspace is expressed entirely in terms of the super-Cotton tensor, see appendix A.

\subsection{Type I minimal supergravity in conventional superspace}

Type I minimal supergravity makes use of two compensators, a covariantly chiral scalar $\Phi$ and its conjugate $\bar{\Phi}$. The chiral compensator is defined to be nowhere vanishing, have $\mathrm{U}(1)_{R}$ charge equal to $-1 / 2$,

$$
\overline{\mathcal{D}}_{\alpha} \Phi=0, \quad J \Phi=-\frac{1}{2} \Phi,
$$

and possess the super-Weyl transformation law

$$
\Phi^{\prime}=\mathrm{e}^{\frac{1}{2} \sigma} \Phi .
$$

In general, the $\mathrm{U}(1)_{R}$ charge of a chiral scalar and its super-Weyl weight are equal in magnitude and opposite in sign [29].

The freedom to perform the super-Weyl and local $\mathrm{U}(1)_{R}$ transformations allows us to choose a gauge $\Phi=1$, which implies the consistency conditions

$$
\mathcal{S}=0, \quad \Phi_{\alpha}=0, \quad \Phi_{a}=-\mathcal{C}_{a} .
$$

This reduces the structure group from $\mathrm{SL}(2, \mathbb{R}) \times \mathrm{U}(1)_{R}$ to its subgroup $\mathrm{SL}(2, \mathbb{R})$. Instead of imposing the gauge condition $\Phi=1$ (which completely fixes the super-Weyl and local $\mathrm{U}(1)_{R}$ freedom), it is more convenient to partially fix the super-Weyl and local $\mathrm{U}(1)_{R}$ symmetry by imposing only the conditions (3.11). The residual super-Weyl and local $\mathrm{U}(1)_{R}$ symmetry is described by transformations which are parametrised in terms of a covariantly chiral scalar parameter $\lambda, \overline{\mathcal{D}}_{\alpha} \lambda=0$, and have the form [33]

$$
\begin{aligned}
\mathcal{D}^{\prime}{ }_{\alpha}= & \mathrm{e}^{\frac{1}{2}(3 \bar{\lambda}-\lambda)}\left(\mathcal{D}_{\alpha}+\mathcal{D}^{\gamma} \lambda M_{\gamma \alpha}\right), \\
\tilde{\mathcal{D}}^{\prime}{ }_{a}= & \mathrm{e}^{\lambda+\bar{\lambda}}\left(\tilde{\mathcal{D}}_{a}-\frac{\mathrm{i}}{2}\left(\gamma_{a}\right)^{\alpha \beta} \mathcal{D}_{\alpha} \lambda \overline{\mathcal{D}}_{\beta}-\frac{\mathrm{i}}{2}\left(\gamma_{a}\right)^{\alpha \beta} \overline{\mathcal{D}}_{\alpha} \bar{\lambda} \mathcal{D}_{\beta}\right. \\
& \left.\quad+\varepsilon_{a b c} \tilde{\mathcal{D}}^{b}(\lambda+\bar{\lambda}) M^{c}-\frac{\mathrm{i}}{2}\left(\mathcal{D}^{\gamma} \lambda\right) \overline{\mathcal{D}}_{\gamma} \bar{\lambda} M_{a}\right),
\end{aligned}
$$

where we have introduced the operator $\tilde{\mathcal{D}}_{a}:=\mathcal{D}_{a}-\mathrm{i} \mathcal{C}_{a} J$. The covariant derivatives $\left(\tilde{\mathcal{D}}_{a}, \mathcal{D}_{\alpha}, \overline{\mathcal{D}}^{\alpha}\right)$ do not contain any $\mathrm{U}(1)_{R}$ connection and obey the algebra

$$
\begin{aligned}
& \left\{\mathcal{D}_{\alpha}, \mathcal{D}_{\beta}\right\}=-4 \overline{\mathcal{R}} M_{\alpha \beta}, \\
& \left\{\mathcal{D}_{\alpha}, \overline{\mathcal{D}}_{\beta}\right\}=-2 \mathrm{i}\left(\gamma^{c}\right)_{\alpha \beta} \tilde{\mathcal{D}}_{c}-2 \varepsilon_{\alpha \beta} \mathcal{C}^{\gamma \delta} M_{\gamma \delta} .
\end{aligned}
$$


The above partially gauge fixed geometric setting is completely suitable to describe type I supergravity and its matter couplings. However, it is not an ideal formalism for reducing the supergravity actions to components. From the point of view of component reduction, it is advantageous to make use of a supergravity formulation with a larger gauge group than that of conventional superspace. Such a framework is provided by the $\mathcal{N}=2$ conformal superspace developed in [30]. Its important features are: (i) it is well adapted to reducing off-shell supergravity-matter actions to components; and (ii) conventional superspace is a gauge fixed version of conformal superspace. The salient details of $\mathcal{N}=2$ conformal superspace are given in appendix A. Below we show how to describe type I supergravity in this setting.

\subsection{The superconformal setting for type I supergravity}

In conformal superspace, the compensator is a primary nowhere vanishing chiral superfield $\Phi$ of dimension $1 / 2$,

$$
\bar{\nabla}_{\alpha} \Phi=0, \quad K_{A} \Phi=0, \quad \mathbb{D} \Phi=\frac{1}{2} \Phi .
$$

The chirality of $\Phi$ fixes its $\mathrm{U}(1)_{R}$ charge, $J \Phi=-\mathbb{D} \Phi$. We define the component fields of $\Phi$ as follows:

$$
\phi:=\Phi\left|, \quad \zeta_{\alpha}:=\nabla_{\alpha} \Phi\right|, \quad \bar{M}=-\frac{1}{4} \nabla^{2} \Phi \mid .
$$

Similar to the $\mathcal{N}=1$ case, one can use the compensator $\Phi$ to introduce dimensionless and $\mathrm{U}(1)_{R}$ neutral covariant derivatives, $\mathscr{D}_{A}=\left(\mathscr{D}_{a}, \mathscr{D}_{\alpha}, \overline{\mathscr{D}}^{\alpha}\right)$, that take every primary superfield to a primary one. They are defined by

$$
\begin{aligned}
\mathscr{D}_{\alpha} & :=\sqrt{\frac{\Phi}{\bar{\Phi}^{3}}}\left(\nabla_{\alpha}-\nabla^{\beta} \ln \Phi M_{\alpha \beta}+\nabla_{\alpha} \ln \Phi J-\nabla_{\alpha} \ln \Phi \mathbb{D}\right), \\
\mathscr{D}_{a} & :=-\frac{\mathrm{i}}{4}\left(\gamma_{a}\right)^{\alpha \beta}\left\{\mathscr{D}_{\alpha}, \overline{\mathscr{D}}_{\beta}\right\} .
\end{aligned}
$$

One can explicitly check that

$$
\mathscr{D}_{A} \Phi=0,
$$

which tells us that when acting on primary superfields the graded commutator $\left[\mathscr{D}_{A}, \mathscr{D}_{B}\right\}$ contains no $\mathrm{U}(1)_{R}$ curvature.

When acting on primary superfields the operators $\mathscr{D}_{A}$ satisfy the algebra

$$
\begin{aligned}
& \left\{\mathscr{D}_{\alpha}, \mathscr{D}_{\beta}\right\}=-4 \mathscr{\mathscr { R }}_{\alpha \beta}, \quad\left\{\overline{\mathscr{D}}_{\alpha}, \overline{\mathscr{D}}_{\beta}\right\}=4 \mathscr{R} M_{\alpha \beta}, \\
& \left\{\mathscr{D}_{\alpha}, \overline{\mathscr{D}}_{\beta}\right\}=-2 \mathrm{i} \mathscr{D}_{\alpha \beta}-2 \varepsilon_{\alpha \beta} \mathscr{C}^{\gamma \delta} M_{\gamma \delta},
\end{aligned}
$$

where we have introduced the primary superfields

$$
\begin{aligned}
\mathscr{R} & :=-\frac{1}{4 \Phi^{3}} \bar{\nabla}^{2} \bar{\Phi}, \\
\mathscr{C}_{\alpha \beta} & :=-\frac{1}{4}\left[\nabla_{(\alpha}, \bar{\nabla}_{\beta)}\right]\left(\frac{1}{\Phi \bar{\Phi}}\right),
\end{aligned}
$$

which are dimensionless and $\mathrm{U}(1)_{R}$ neutral. The algebra of covariant derivatives (3.18) formally coincides with (3.13). 
We can relate the superconformal framework to the one of conventional superspace by gauge fixing the additional symmetries. We can use the conformal boosts and $S$ supersymmetry transformations to impose the gauge condition

$$
B_{A}=0,
$$

which degauges conformal superspace to conventional superspace [30]. The composites (3.19) become the following super-Weyl invariant objects

$$
\begin{aligned}
\mathscr{C}_{\alpha \beta} & =-\frac{1}{4}\left(\left[\mathcal{D}_{(\alpha}, \overline{\mathcal{D}}_{\beta)}\right]-4 \mathcal{C}_{\alpha \beta}\right) \frac{1}{\Phi \bar{\Phi}}, \\
\mathscr{R} & =-\frac{1}{4 \Phi^{3}}\left(\overline{\mathcal{D}}^{2}-4 R\right) \bar{\Phi}
\end{aligned}
$$

while the super-Cotton tensor of conformal superspace $W_{\alpha \beta}$ coincides with (3.6). Upon imposing the additional gauge condition

$$
\Phi=1
$$

the composites (3.19) coincide with the torsion components $\mathcal{R}$ and $\mathcal{C}_{\alpha \beta}$.

It is also worth mentioning that one can use the compensator to construct super-Weyl invariant covariant derivatives $\mathcal{D}_{A}^{(\Phi)}$ in conventional superspace as follows:

$$
\begin{aligned}
& \mathcal{D}_{\alpha}^{(\Phi)}=\sqrt{\frac{\Phi}{\bar{\Phi}^{3}}}\left(\mathcal{D}_{\alpha}-\mathcal{D}^{\beta} \ln \Phi M_{\alpha \beta}+\mathcal{D}_{\alpha} \ln \Phi J\right), \\
& \mathcal{D}_{a}^{(\Phi)}=-\frac{\mathrm{i}}{4}\left(\gamma_{a}\right)^{\alpha \beta}\left\{\mathcal{D}_{\alpha}^{(\Phi)}, \mathcal{D}_{\beta}^{(\Phi)}\right\} .
\end{aligned}
$$

It can be checked that these covariant derivatives satisfy the same algebra as (3.18). Unlike the operators $\mathscr{D}_{A}$, eq. (3.16), they do not annihilate the compensator $\Phi$.

Many supergravity models may be constructed as integrals over $\mathcal{N}=2$ superspace of the form

$$
S=\int \mathrm{d}^{3 \mid 4} z E \mathcal{L}
$$

where $\mathcal{L}$ is a real primary superfield of dimension 1 . One can also use the chiral action principle

$$
S_{\mathrm{c}}=\int \mathrm{d}^{3 \mid 2} z_{\mathrm{c}} \mathcal{E} \mathcal{L}_{\mathrm{c}}, \quad \mathrm{d}^{3 \mid 2} z_{\mathrm{c}}:=\mathrm{d}^{3} x \mathrm{~d}^{2} \theta,
$$

where $\mathcal{L}_{\mathrm{c}}$ is a primary chiral scalar of dimension 2 and $\mathcal{E}$ denotes the chiral density. ${ }^{11}$ Every action (3.24) can be rewritten as a chiral action because of the relation [29]

$$
\int \mathrm{d}^{3 \mid 4} z E \mathcal{L}=-\frac{1}{4} \int \mathrm{d}^{3 \mid 2} z_{c} \mathcal{E} \bar{\nabla}^{2} \mathcal{L}_{\mathrm{c}}
$$

The chiral action can be reduced to components using the component reduction formula $[18,19]$

$$
S_{\mathrm{c}}=-\frac{1}{4} \int \mathrm{d}^{3} x e\left[\nabla^{2}-2 \mathrm{i}\left(\gamma^{a}\right)_{\alpha \beta} \bar{\psi}_{a}^{\alpha} \nabla^{\beta}-2 \varepsilon^{a b c}\left(\gamma_{a}\right)_{\alpha \beta} \bar{\psi}_{b}{ }^{\alpha} \bar{\psi}_{c}{ }^{\beta}\right] \mathcal{L}_{\mathrm{c}} \mid,
$$

\footnotetext{
${ }^{11}$ The explicit expression for $\mathcal{E}$ in terms of the supergravity prepotentials is given in [68].
} 
where $\psi_{a}{ }^{\beta}=e_{a}{ }^{m} \psi_{m}{ }^{\beta}$ and $\bar{\psi}_{a}{ }^{\beta}=e_{a}{ }^{m} \bar{\psi}_{m}{ }^{\beta}$ denote the gravitini. The component fields of the Weyl multiplet were defined in [31]. The vielbein $e_{m}{ }^{a}$, the gravitini $\psi_{m}{ }^{\alpha}$ and $\bar{\psi}_{m}{ }^{\beta}$, the $\mathrm{U}(1)_{R}$ gauge field $V_{m}$ and the dilatation gauge field $b_{m}$ are defined as the lowest components of their corresponding superforms,

$$
e_{m}{ }^{a}:=E_{m}{ }^{a}\left|, \quad \psi_{m}{ }^{\alpha}:=2 E_{m}{ }^{\alpha}\right|, \quad V_{m}:=\Phi_{m}\left|, \quad b_{m}:=B_{m}\right| .
$$

At the component level we will be mainly concerned with bosonic fields.

Using the above results one can construct general actions of the form

$$
S=\int \mathrm{d}^{3 \mid 4} z E \Phi \bar{\Phi} \mathfrak{L}(\mathscr{T}, \mathscr{D} \mathscr{T}, \cdots)
$$

where $\mathfrak{L}$ is a dimensionless superfield constructed out of the torsion components $\mathscr{T}$ and their covariant derivatives $\mathscr{D}_{A}$. As for $\mathcal{N}=1$, we will focus on actions that in components involve at most curvature squared terms.

To fix the additional symmetries in our invariants one can make use of the gauge conditions (3.20) and (3.22) which correspond to the following conditions at the component level

$$
\phi=1, \quad \zeta_{\alpha}=0, \quad b_{m}=0 .
$$

The first condition fixes the dilatations and $\mathrm{U}(1)_{R}$ transformations, the second fixes the $S$-supersymmetry transformations and the last fixes the conformal boosts.

Some useful results for identifying curvature terms in the actions we construct are given below with the gauge conditions (3.30) (compare with (2.27)):

$$
\begin{aligned}
\square \phi & =\frac{1}{8} R+\text { fermion terms }, \\
\square^{2} \frac{1}{\phi} & =R^{a b} R_{a b}-\frac{23}{64} R^{2}-\frac{1}{8} D^{a} D_{a} R+\text { fermion terms }
\end{aligned}
$$

where we have defined

$$
\begin{aligned}
\square \phi & :=\nabla^{a} \nabla_{a} \Phi \mid \\
\square^{2} \frac{1}{\phi} & :=\nabla^{a} \nabla_{a} \nabla^{b} \nabla_{b} \frac{1}{\Phi} \mid
\end{aligned}
$$

and introduced the covariant derivative

$$
D_{a}=e_{a}^{m}\left(\partial_{m}-\frac{1}{2} \omega_{m}^{b c} M_{b c}-\mathrm{i} V_{m} J-b_{m} \mathbb{D}\right) .
$$

\subsection{Supergravity invariants}

In this subsection we construct supergravity invariants in superspace by making use of the composites (3.19). 


\subsubsection{The supergravity action}

The type I minimal supergravity action with a cosmological term was given in [18] in conventional superspace. It is straightforward to lift the action to conformal superspace and is given by

$$
S=\frac{1}{\kappa} S_{\mathrm{SG}}+\left(\lambda S_{\mathrm{cos}}+\text { c.c. }\right)
$$

where

$$
\begin{aligned}
& S_{\mathrm{SG}}=-4 \int \mathrm{d}^{3 \mid 4} z E \bar{\Phi} \Phi, \\
& S_{\mathrm{cos}}=\int \mathrm{d}^{3 \mid 2} z \mathcal{E} \Phi^{4}
\end{aligned}
$$

and $\lambda$ is the cosmological constant, which can be complex in general. The above action contains the Einstein-Hilbert term, $-\frac{1}{2} R$, since one finds the term $-4 \phi \square \bar{\phi}$ at the component level in the action. The detailed component analysis for type I supergravity can be found in [18].

\subsubsection{The $R M^{n}$ invariants}

A locally supersymmetric invariant containing a $R M^{n}$ term is given by

$$
S_{R M^{n}}=\int \mathrm{d}^{3 \mid 4} z E \Phi \bar{\Phi} \mathscr{R}^{n}=\int \mathrm{d}^{3 \mid 2} z \mathcal{E} \mathscr{R}^{n+1} \Phi^{4} .
$$

The corresponding invariant at the component level contains a term proportional to $\left(M / \phi^{3}\right)^{n} \phi \square \bar{\phi}$, which upon gauge fixing gives rise to the term

$$
\frac{(n+1)}{8} R M^{n}
$$

The component action at the bosonic level was explicitly given in [23].

\subsubsection{The scalar curvature squared invariant}

A scalar curvature squared invariant is described by

$$
S_{\text {scalar }^{2}}=-4 \int \mathrm{d}^{3 \mid 4} z E \Phi \bar{\Phi} \mathscr{R} \overline{\mathscr{R}}=-\frac{1}{4} \int \mathrm{d}^{3 \mid 4} z E \frac{1}{(\Phi \bar{\Phi})^{2}}\left(\nabla^{2} \Phi\right) \bar{\nabla}^{2} \bar{\Phi} .
$$

One can show that the above invariant will involve a term proportional to $1 /(\phi \bar{\phi})^{2} \square \phi \square \bar{\phi}$, which gives rise to the scalar curvature squared term $-\frac{1}{16} R^{2}$ at the component level. One can check that the action also contains a $|M|^{4}$ term. At the component level the explicit bosonic action was explicitly given in [23].

\subsubsection{The Ricci curvature squared invariant}

An invariant containing a Ricci squared term is given by

$$
S_{\mathrm{Ricci}^{2}}=4 \int \mathrm{d}^{3 \mid 4} z E \Phi \bar{\Phi}^{\alpha \beta} \mathscr{C}_{\alpha \beta} .
$$


The above action also can be seen to contain a scalar curvature squared term at the component level by making use of the results in [18]. It therefore makes sense to introduce the one parameter family of invariants

$$
S_{\zeta}=4 \int \mathrm{d}^{3 \mid 4} z E \Phi \bar{\Phi}\left(\mathscr{C}^{\alpha \beta} \mathscr{C}_{\alpha \beta}+\zeta \mathscr{R} \overline{\mathscr{R}}\right)
$$

where $\zeta$ parametrizes the scalar curvature squared contribution.

It should be mentioned that an alternative invariant containing a Ricci curvature squared term may be constructed and is given by

$$
S_{\Xi}=-\frac{1}{8} \int \mathrm{d}^{3 \mid 2} z_{c} \mathcal{E} \frac{\Xi}{\Phi}+\text { c.c. },
$$

where we have defined

$$
\Xi=\nabla^{a} \nabla_{a} \bar{\nabla}^{2} \frac{1}{\bar{\Phi}}=\nabla^{a} \bar{\nabla}^{2} \nabla_{a} \frac{1}{\bar{\Phi}}=\bar{\nabla}^{2} \nabla^{a} \nabla_{a} \frac{1}{\bar{\Phi}}
$$

Remarkably, one can check that the superfield $\Xi$ is both chiral and primary. It corresponds to the dimension 5/2 composite constructed at the bosonic level in [23]. The fermionic terms may be recovered by straightforward component reduction of our result. It is also worth noting that the invariant (3.42) can be written in terms of the conventional superspace formulation of [29] as follows:

$$
\begin{aligned}
S_{\Xi}= & -\frac{1}{4} \int \mathrm{d}^{3 \mid 4} z E\left\{\frac{1}{\Phi} \mathcal{D}^{\alpha \beta} \mathcal{D}_{\alpha \beta} \frac{1}{\bar{\Phi}}+\frac{11 \mathcal{C}^{\alpha \beta} \mathcal{C}_{\alpha \beta}}{4 \Phi \bar{\Phi}}+\frac{6 \mathcal{R} \overline{\mathcal{R}}}{\Phi \bar{\Phi}}+8 \mathcal{C}^{\alpha \beta}\left(\mathcal{D}_{\alpha} \frac{1}{\Phi}\right) \overline{\mathcal{D}}_{\beta} \frac{1}{\bar{\Phi}}\right. \\
& -\frac{8 \mathrm{i}}{\Phi^{2} \bar{\Phi}} \mathcal{C}^{\alpha \beta} \mathcal{D}_{\alpha \beta} \frac{1}{\bar{\Phi}}-\frac{13 \mathrm{i}}{\Phi} \mathcal{C}^{\alpha \beta} \mathcal{D}_{\alpha \beta} \frac{1}{\bar{\Phi}}-\frac{6 \mathcal{S}^{2}}{\Phi \bar{\Phi}}-\frac{3 \mathrm{i}}{2 \Phi \bar{\Phi}} \mathcal{D}^{\alpha \beta} \mathcal{C}_{\alpha \beta}+\frac{9 \bar{R}}{2 \Phi} \overline{\mathcal{D}}^{2} \frac{1}{\bar{\Phi}} \\
& \left.-\frac{6 \mathrm{i}}{\Phi \bar{\Phi}} \mathcal{D}^{\alpha} \overline{\mathcal{D}}_{\alpha} \mathcal{S}-\frac{6 \bar{R} \bar{\Phi}^{2}}{\Phi^{2}}\left(\overline{\mathcal{D}}_{\alpha} \frac{1}{\bar{\Phi}}\right) \overline{\mathcal{D}}^{\alpha} \frac{1}{\bar{\Phi}}\right\}+ \text { c.c. }
\end{aligned}
$$

Note that in the gauge where $\Phi=1$ we have

$$
\mathcal{S}=0, \quad \mathcal{D}_{\alpha \beta} \Phi=-\frac{\mathrm{i}}{2} \mathcal{C}_{\alpha \beta}
$$

and the action (3.44) simply becomes

$$
S_{\Xi}=4 \int \mathrm{d}^{3 \mid 4} z E\left(\mathcal{C}^{\alpha \beta} \mathcal{C}_{\alpha \beta}-\frac{3}{4} \mathcal{R} \overline{\mathcal{R}}\right)
$$

It follows that the action (3.42) coincides with $S_{-3 / 4}$.

Upon reducing to components and imposing the gauge conditions (3.20) and (3.22), the action (3.46) gives rise to the following combination of Ricci and scalar curvature squared terms:

$$
R^{a b} R_{a b}-\frac{23}{64} R^{2}
$$

A pure Ricci curvature squared invariant can be identified and is simply given by

$$
S_{\mathrm{Ricci}^{2}}^{(\text {pure }}=S_{5}=4 \int \mathrm{d}^{3 \mid 4} z E \Phi \bar{\Phi}\left(\mathscr{C}^{\alpha \beta} \mathscr{C}_{\alpha \beta}+5 \mathscr{R} \overline{\mathscr{R}}\right) \text {. }
$$


In the above we have restricted our attention to curvature squared terms. However, our approach naturally provides a means to address locally supersymmetric functionals containing higher powers of the curvature tensor and its covariant derivatives. One can simply consider other actions of the form (3.29), which involves covariant derivatives of the primary superfields (3.19). Amongst the descendants of (3.19) it is worth mentioning the following rank 2 symmetric spinor

$$
\mathscr{W}_{\alpha \beta}=-\frac{\mathrm{i}}{4}\left[\mathscr{D}^{\gamma}, \overline{\mathscr{D}}_{\gamma}\right] \mathscr{C}_{\alpha \beta},
$$

which is related to the super-Cotton tensor $W_{\alpha \beta}$ by the rule

$$
W_{\alpha \beta}=(\Phi \bar{\Phi})^{2} \mathscr{W}_{\alpha \beta}
$$

The super-Cotton tensor is independent of the compensator $\Phi$ and satisfies the condition

$$
\nabla^{\beta} W_{\alpha \beta}=0
$$

\subsection{Models for massive supergravity}

The invariants in the previous section are useful building blocks in the construction of massive supergravity. In this section we analyse the dynamics of a general supergravity model and determine the conditions in which we have massive supergravity.

We consider the supergravity model ${ }^{12}$

$$
S=\frac{1}{\kappa} S_{\mathrm{SG}}+\mu_{2} S_{\mathrm{scalar}^{2}}+\mu_{3} S_{\mathrm{Ricci}^{2}}+\frac{1}{\tilde{\mu}} S_{\mathrm{CSG}}+\left(\lambda S_{\mathrm{cos}}+\mu_{0} S_{M^{3}}+\mu_{1} S_{M^{4}}+\text { c.c. }\right) .
$$

Here $\lambda, \mu_{0}$ and $\mu_{1}$ are allowed to be complex in general. The action for $\mathcal{N}=2$ conformal supergravity, $S_{\mathrm{CSG}}$, was originally constructed in [35]. Within the conformal superspace approach [30], its construction was given in [31]. In what follows we assume $\mu_{3} \geq 0$ as in [23].

Varying the action (3.52) with respect to the compensator $\Phi$ leads to the equation of motion

$$
\begin{aligned}
0= & 4 \lambda-\frac{4}{\kappa} \mathscr{R}-2 \mu_{0} \mathscr{R}^{2}-\frac{1}{2} \bar{\mu}_{0}\left(\overline{\mathscr{D}}^{2}-4 \mathscr{R}\right) \overline{\mathscr{R}}-5 \mu_{1} \mathscr{R}^{3}-\frac{3}{4} \bar{\mu}_{1}\left(\overline{\mathscr{D}}^{2}-4 \mathscr{R}\right) \overline{\mathscr{R}}^{2} \\
& +\frac{1}{4}\left(\mu_{3}-\mu_{2}\right)\left(\overline{\mathscr{D}}^{2}-4 \mathscr{R}\right)\left(\mathscr{D}^{2}-4 \overline{\mathscr{R}}\right) \mathscr{R}+\left(\mu_{3}-2 \mu_{2}\right) \mathscr{R}\left(\overline{\mathscr{D}}^{2}-4 \mathscr{R}\right) \overline{\mathscr{R}} \\
& -2 \mu_{3}\left(\overline{\mathscr{D}}^{2}-4 \mathscr{R}\right)\left(\mathscr{C}^{a} \mathscr{C}_{a}\right) .
\end{aligned}
$$

As in the $\mathcal{N}=1$ case there is no contribution proportional to $\tilde{\mu}^{-1}$ since the conformal supergravity action is independent of the compensator. The equation of motion for the conformal supergravity prepotential is

$$
\frac{1}{\mu} W_{\alpha \beta}+T_{\alpha \beta}=0
$$

\footnotetext{
${ }^{12}$ The class of supergravity models considered here is more general than those considered in [23] because we have allowed some of the coupling constants to be complex.
} 
where $\mu$ is related to $\tilde{\mu}$ by a multiplicative constant and the supercurrent $T_{\alpha \beta}$ is

$$
\begin{aligned}
(\Phi \bar{\Phi})^{-2} T_{\alpha \beta}= & \frac{1}{\kappa} \mathbb{T}_{\alpha \beta}^{(\mathrm{SG})}+\mu_{2} \mathbb{T}_{\alpha \beta}^{\left(\mathrm{sca}^{2}\right)}+\mu_{3} \mathbb{T}^{\left(\mathrm{Ric}^{2}\right)}{ }_{\alpha \beta} \\
& +\left(\mu_{0} \mathbb{T}_{\alpha \beta}^{\left(M^{3}\right)}+\mu_{1} \mathbb{T}^{\left(M^{4}\right)}{ }_{\alpha \beta}+\text { c.c. }\right)
\end{aligned}
$$

where

$$
\begin{aligned}
\mathbb{T}^{(\mathrm{SG})}{ }_{\alpha \beta}= & -\frac{1}{2} \mathscr{C}_{\alpha \beta}, \\
\mathbb{T}^{\left(M^{n+2}{ }_{\alpha \beta}=\right.} & -\frac{n+1}{8}\left(\left[\mathscr{D}_{(\alpha}, \overline{\mathscr{D}}_{\beta)}\right]+4 \mathscr{C}_{\alpha \beta}\right) \mathscr{R}^{n} \\
\mathbb{T}^{\left(\mathrm{sca}^{2}{ }_{\alpha \beta}=\right.} & -\frac{1}{8}\left(\left[\mathscr{D}_{(\alpha}, \overline{\mathscr{D}}_{\beta)}\right]+4 \mathscr{C}_{\alpha \beta}\right)\left(\left(\mathscr{D}^{2}-4 \overline{\mathscr{R}}\right) \mathscr{R}+\left(\overline{\mathscr{D}}^{2}-4 \mathscr{R}\right) \overline{\mathscr{R}}-4 \mathscr{R} \overline{\mathscr{R}}\right) \\
& -2\left(\mathscr{D}_{(\alpha} \mathscr{R}\right) \overline{\mathscr{D}}_{\beta)} \overline{\mathscr{R}} \\
\mathbb{T}^{\left(\mathrm{Ric}^{2}{ }_{\alpha \beta}=\right.} & \frac{\mathrm{i}}{2}\left[\mathscr{D}^{\delta}, \overline{\mathscr{D}}_{\delta}\right] \mathscr{W}_{\alpha \beta}-\frac{1}{8}\left[\mathscr{D}_{(\alpha}, \overline{\mathscr{D}}_{\beta)}\right]\left(\mathscr{D}^{2} \mathscr{R}+\overline{\mathscr{D}}^{2} \overline{\mathscr{R}}\right)+\frac{3}{2}\left[\mathscr{D}_{(\alpha}, \overline{\mathscr{D}}_{\beta)}\right]\left(\mathscr{C}^{\gamma \delta} \mathscr{C}_{\gamma \delta}\right) \\
& +\frac{5}{6} \mathscr{C}_{\alpha \beta}\left(\mathscr{D}^{2} \mathscr{R}+\overline{\mathscr{D}}^{2} \overline{\mathscr{R}}\right)+2 \mathrm{i} \mathscr{C}^{\gamma \delta}\left(\overline{\mathscr{D}}_{(\alpha} \mathscr{C}_{\beta \gamma \delta)}+\mathscr{D}_{(\alpha} \overline{\mathscr{C}}_{\beta \gamma \delta)}\right) \\
& +\frac{2 \mathrm{i}}{3}\left(\overline{\mathscr{D}}^{\gamma} \overline{\mathscr{R}}\right) \mathscr{C}_{\alpha \beta \gamma}+\frac{2 \mathrm{i}}{3}\left(\mathscr{D}^{\gamma} \mathscr{R}\right) \overline{\mathscr{C}}_{\alpha \beta \gamma}+\frac{20}{9}\left(\mathscr{D}_{(\alpha} \mathscr{R}\right) \overline{\mathscr{D}}_{\beta)} \overline{\mathscr{R}} \\
& +2 \mathscr{C}_{\alpha \beta} \mathscr{C}^{\gamma \delta} \mathscr{C}_{\gamma \delta}-8 \mathscr{C}_{(\alpha}{ }^{\gamma \delta} \overline{\mathscr{C}}_{\beta) \gamma \delta}-8 \mathscr{C}_{\alpha \beta} \mathscr{R} \mathscr{\mathscr { R }} .
\end{aligned}
$$

Here we have defined

$$
\mathscr{C}_{\alpha \beta \gamma}=-\mathrm{i} \mathscr{D}_{(\alpha} \mathscr{C}_{\beta \gamma)} .
$$

One can check the supergravity equation of motion (3.54) by making use of the results for the deformation of the prepotential in appendix $\mathrm{C}$, which imply

$$
\delta S_{\mathrm{CSG}}=\int \mathrm{d}^{3 \mid 4} z E \delta H^{\alpha \beta} W_{\alpha \beta}, \quad \delta\left[S-\frac{1}{\tilde{\mu}} S_{\mathrm{CSG}}\right]=\int \mathrm{d}^{3 \mid 4} z E \delta H^{\alpha \beta} T_{\alpha \beta} .
$$

The supercurrent $T_{\alpha \beta}$ obeys the conservation equation

$$
\nabla^{\beta} T_{\alpha \beta}=0
$$

when the compensator obeys its equation of motion (3.53).

The supergravity equations (3.53) and (3.54) are automatically super-Weyl invariant. Upon imposing the gauge (3.20) and reducing to conventional superspace, the local superWeyl and $\mathrm{U}(1)_{R}$ symmetries may be fixed by imposing the gauge

$$
\Phi=1 .
$$

This is equivalent to making the replacements $\mathscr{D}_{A} \rightarrow \mathcal{D}_{A}, \mathscr{C}_{\alpha \beta} \rightarrow \mathcal{C}_{\alpha \beta}$ and $\mathscr{R} \rightarrow \mathcal{R}$ everywhere. The super-Weyl invariance can be restored by making the inverse replacement. In what follows we will assume the gauge condition (3.60).

We are interested in maximally supersymmetric solutions of the supergravity equations of motion. In type I supergravity backgrounds, all maximally supersymmetric backgrounds $[18,65]$ are characterised by dimension- 1 torsion superfields under the following constraints

$$
\mathcal{S}=0, \quad \mathcal{R C}_{a}=0, \quad \mathcal{D}_{A} \mathcal{R}=0, \quad \mathcal{D}_{A} \mathcal{C}_{b}=0
$$


The complete algebra of covariant derivatives is

$$
\begin{aligned}
\left\{\mathcal{D}_{\alpha}, \mathcal{D}_{\beta}\right\} & =-4 \overline{\mathcal{R}} M_{\alpha \beta}, \\
\left\{\mathcal{D}_{\alpha}, \overline{\mathcal{D}}_{\beta}\right\} & =-2 \mathrm{i}\left(\gamma^{c}\right)_{\alpha \beta}\left(\mathcal{D}_{c}-\mathrm{i} \mathcal{C}_{c} J\right)+4 \varepsilon_{\alpha \beta} \mathcal{C}^{c} M_{c}, \\
{\left[\mathcal{D}_{a}, \mathcal{D}_{\beta}\right] } & =\mathrm{i} \varepsilon_{a b c}\left(\gamma^{b}\right)_{\beta}{ }^{\gamma} \mathcal{C}^{c} \mathcal{D}_{\gamma}-\mathrm{i}\left(\gamma_{a}\right)_{\beta \gamma} \overline{\mathcal{R}} \overline{\mathcal{D}}^{\gamma} \\
{\left[\mathcal{D}_{a}, \mathcal{D}_{b}\right] } & =4 \varepsilon_{a b c}\left(\mathcal{C}^{c} \mathcal{C}_{d}+\delta^{c}{ }_{d} \overline{\mathcal{R}} \mathcal{R}\right) M^{d}
\end{aligned}
$$

The equations of motion (3.53) and (3.54) simplify significantly for maximally supersymmetric backgrounds where we have the conditions

$$
\mathcal{C}_{a}=0, \quad \mathcal{R}=\mathcal{R}_{0}=\text { const } .
$$

In this case the supercurrent and super-Cotton tensor vanish,

$$
T_{\alpha \beta}=W_{\alpha \beta}=0,
$$

which means that (3.54) is identically satisfied while the equation on the compensator reduces to

$$
0=4 \lambda-\frac{4}{\kappa} \mathcal{R}_{0}-2 \mu_{0} \mathcal{R}_{0}^{2}+2 \bar{\mu}_{0} \mathcal{R}_{0} \overline{\mathcal{R}}_{0}-5 \mu_{1} \mathcal{R}_{0}^{3}+3 \bar{\mu}_{1} \mathcal{R}_{0} \overline{\mathcal{R}}_{0}^{2}+4 \mu_{2} \mathcal{R}_{0}^{2} \overline{\mathcal{R}}_{0}
$$

We now consider perturbations in the model (3.52) about the maximally supersymmetric solution:

$$
\mathcal{R}=\mathcal{R}_{0}+\Delta \mathcal{R}, \quad \mathcal{C}_{\alpha \beta}=\Delta \mathcal{C}_{\alpha \beta} .
$$

The equation of motion on the compensator becomes

$$
\begin{aligned}
0= & \left(-\frac{4}{\kappa}-4 \mu_{0} \mathcal{R}_{0}+2 \bar{\mu}_{0} \overline{\mathcal{R}}_{0}-15 \mu_{1} \mathcal{R}_{0}^{2}+3 \bar{\mu}_{1} \overline{\mathcal{R}}_{0}^{2}+8 \mu_{2} \mathcal{R}_{0} \overline{\mathcal{R}}_{0}\right) \Delta \mathcal{R} \\
& +\left(2 \mathcal{R}_{0}\left(\bar{\mu}_{0}+3 \bar{\mu}_{1} \overline{\mathcal{R}}_{0}\right)+4 \mu_{2} \mathcal{R}_{0}^{2}\right) \Delta \overline{\mathcal{R}} \\
& +\left(-\frac{1}{2}\left(\bar{\mu}_{0}+3 \bar{\mu}_{1} \overline{\mathcal{R}}_{0}\right)-\mu_{2} \mathcal{R}_{0}\right) \overline{\mathcal{D}}^{2} \Delta \overline{\mathcal{R}} \\
& +\left(\mu_{2}-\mu_{3}\right) \mathcal{R}_{0} \mathcal{D}^{2} \Delta \mathcal{R}-\frac{1}{4}\left(\mu_{2}-\mu_{3}\right) \overline{\mathcal{D}}^{2} \mathcal{D}^{2} \Delta \mathcal{R} .
\end{aligned}
$$

Thus we see that $\Delta \mathcal{R}$ is propagating in general. However one can simplify the equation of motion (3.67) by turning it into an algebraic one by setting

$$
\begin{aligned}
\mu_{2} & =\mu_{3}, \\
0 & =\bar{\mu}_{0}+3 \bar{\mu}_{1} \overline{\mathcal{R}}_{0}+2 \mu_{2} \mathcal{R}_{0} .
\end{aligned}
$$

The generic case is characterized by the condition

$$
\frac{1}{\kappa}+\frac{3}{4}\left(\mu_{1} \mathcal{R}_{0}^{2}+\bar{\mu}_{1} \overline{\mathcal{R}}_{0}^{2}\right)-3 \mu_{2}\left|\mathcal{R}_{0}\right|^{2} \neq 0,
$$


which requires $\Delta \mathcal{R}=\Delta \overline{\mathcal{R}}=0$. Then the supergravity equation of motion reduces to

$$
\begin{gathered}
-\frac{\mu_{3}}{8}\left[\mathcal{D}^{\gamma}, \overline{\mathcal{D}}_{\gamma}\right]\left[\mathcal{D}^{\delta}, \overline{\mathcal{D}}_{\delta}\right] \Delta \mathcal{C}_{\alpha \beta}+\frac{\mathrm{i}}{4 \mu}\left[\mathcal{D}^{\gamma}, \overline{\mathcal{D}}_{\gamma}\right] \Delta \mathcal{C}_{\alpha \beta} \\
+\left(\frac{1}{2 \kappa}-\frac{3}{2} \mu_{1} \mathcal{R}_{0}^{2}-\frac{3}{2} \bar{\mu}_{1} \overline{\mathcal{R}}_{0}^{2}\right) \Delta \mathcal{C}_{\alpha \beta}=0,
\end{gathered}
$$

which gives

$$
\mu_{3} \mathcal{D}^{b} \mathcal{D}_{b} \Delta \mathcal{C}_{a}+\frac{1}{2 \mu} \varepsilon_{a b c} \mathcal{D}^{b} \Delta \mathcal{C}^{c}+\left(\frac{1}{4 \kappa}+8 \mu_{3}\left|\mathcal{R}_{0}\right|^{2}-\frac{3}{4}\left(\mu_{1} \mathcal{R}_{0}^{2}+\bar{\mu}_{1} \overline{\mathcal{R}}_{0}^{2}\right)\right) \Delta \mathcal{C}_{a}=0
$$

By linearizing the Bianchi identity (3.4) about the background chosen one can see that $\Delta \mathcal{C}_{\alpha \beta}$ is divergenceless $\mathcal{D}^{\beta} \Delta \mathcal{C}_{\alpha \beta}=0$.

In general for a symmetric spinor $T_{\alpha_{1} \cdots \alpha_{n}}=T_{\left(\alpha_{1} \cdots \alpha_{n}\right)}$ that is divergenceless,

$$
\mathcal{D}^{\alpha} T_{\alpha \alpha_{1} \cdots \alpha_{n-1}}=0,
$$

one can check the following identity holds in the background chosen

$$
\mathcal{D}^{\beta} \mathcal{D}^{\gamma} \overline{\mathcal{D}}_{\gamma} T_{\alpha_{1} \cdots \alpha_{n-1} \beta}=\overline{\mathcal{D}}^{\beta} \mathcal{D}^{\gamma} \overline{\mathcal{D}}_{\gamma} T_{\alpha_{1} \cdots \alpha_{n-1} \beta}=0 .
$$

This implies that the operator $\frac{\mathrm{i}}{2} \mathcal{D}^{\gamma} \overline{\mathcal{D}}_{\gamma}$ preserves the divergenceless condition of the superfield $\Delta \mathcal{C}_{\alpha \beta}$.

In the case $\mu_{3} \neq 0$ we may write the equation of motion in the following factorized form

$$
\left(\frac{\mathrm{i}}{2} \mathcal{D}^{\gamma} \overline{\mathcal{D}}_{\gamma}+m_{-}\right)\left(\frac{\mathrm{i}}{2} \mathcal{D}^{\delta} \overline{\mathcal{D}}_{\delta}-m_{+}\right) \Delta \mathcal{C}_{\alpha \beta}=0
$$

where

$$
\begin{aligned}
m_{+}-m_{-} & =-\frac{1}{2 \mu \mu_{3}}, \\
m_{+} m_{-} & =-\frac{1}{4 \mu_{3}}\left(\frac{1}{\kappa}-3 \mu_{1} \mathcal{R}_{0}^{2}-3 \bar{\mu}_{1} \overline{\mathcal{R}}_{0}^{2}\right) .
\end{aligned}
$$

The constants $m_{+}$and $m_{-}$are real for

$$
\frac{1}{4 \mu^{2} \mu_{3}} \geq\left(\frac{1}{\kappa}-3 \mu_{1} \mathcal{R}_{0}^{2}-3 \bar{\mu}_{1} \overline{\mathcal{R}}_{0}^{2}\right) .
$$

The supergravity model (3.52) leads to massive supergravity for different choices of parameters. For instance, we can see from eq. (3.76) that in a Minkowski background with $\mu_{3}>0$ and $\mu=\infty$ we must have either a negative Einstein-Hilbert term $(\kappa<0)$ or no Einstein Hilbert term $(\kappa \rightarrow \infty)$ for massive supergravity.

In the case where $\mu_{3}=0$ and $\mu$ is finite we have the equation

$$
\left(\frac{\mathrm{i}}{2} \mathcal{D}^{\gamma} \overline{\mathcal{D}}_{\gamma}-\hat{m}\right) \Delta \mathcal{C}_{\alpha \beta}=0
$$


where $\hat{m}$ is given by

$$
\hat{m}=-\mu\left(\frac{1}{\kappa}-\frac{3}{2} \mu_{1} \mathcal{R}_{0}^{2}-\frac{3}{2} \bar{\mu}_{1} \overline{\mathcal{R}}_{0}^{2}\right) .
$$

When $\mu_{0}=\mu_{1}=0$ we have topologically massive supergravity.

One should note that the supergravity model (3.52) is more general than the one considered in [23] since the model contains 9 real free parameters. ${ }^{13}$ This leads to an important consequence. In contrast to [23] we have shown that we can eliminate the degrees of freedom associated with the torsion superfield $\mathcal{R}$ in any AdS background satisfying eq. (3.65) that otherwise propagates since $\mathcal{R}$ is coupled to a product of propagating fields. Its elimination can be seen to coincide with removing the contribution from the $R(M+\bar{M})^{2}$ and $R(M-\bar{M})^{2}$ terms in the component action to the linearized equation of motion. These terms contribute to highly non-linear interactions upon imposing the equation of motion on $M$. In [23] the $R(M+\bar{M})^{2}$ term was eliminated by a choice of constraints, which coincides with $\mu_{0}=0$, eq. (3.68a) and $\mu_{1}=-\frac{2}{3} \mu_{2}$. Imposing these constraints and expanding about a background with $\mathcal{R}_{0}=\overline{\mathcal{R}}_{0}$ we see that $(3.68 \mathrm{~b})$ is identically satisfied and one recovers the factorisation (3.73). However, it is important to note that the factorisation holds for the model defined by the constraints (3.68a) and (3.68b) about any AdS background satisfying eq. (3.65).

It is worth mentioning that there exists other type I maximally supersymmetric backgrounds [18] defined by the conditions

$$
\mathcal{R}=0, \quad \mathcal{C}_{a}=\text { const }
$$

In this case the equation on the compensator reduces to

$$
\lambda=0
$$

while the supergravity equation of motion fixes $\mathcal{C}^{2}:=\mathcal{C}^{a} \mathcal{C}_{a}$ as follows

$$
\mathcal{C}^{2}=\frac{1}{8 \kappa \mu_{3}}
$$

We do not discuss linearization about this background here.

\section{$4 \mathcal{N}=2$ supergravity models with a real linear compensator}

The $\mathcal{N}=2$ conventional superspace formulation was presented in the previous section where it was also shown how to describe type I minimal supergravity with the use of a chiral compensator and its conjugate. Type II minimal supergravity, which makes use of a real linear compensator, can be described similarly with conventional superspace. In this section we show how to do this and generalise the geometric framework to a superconformal setting.

\footnotetext{
${ }^{13}$ The cosmological constant can be made to be real via a rescaling of the chiral compensator, $\Phi(z) \rightarrow \mathrm{e}^{\mathrm{i} \alpha} \Phi(z)$.
} 


\subsection{Type II minimal supergravity in conventional superspace}

Type II minimal supergravity makes use of a real linear compensator $G$. The compensator $G$ is defined to be nowhere vanishing and satisfy the following constraint

$$
\left(\mathcal{D}^{2}-4 \overline{\mathcal{R}}\right) G=0 .
$$

The superfield $G$ transforms homogeneously under super-Weyl transformations,

$$
G^{\prime}=\mathrm{e}^{\sigma} G
$$

Since $G$ is nowhere vanishing the super-Weyl transformations permit us to choose a gauge where $G=1$, which leads to the consistency condition

$$
\mathcal{R}=0 .
$$

We may refer to the superspace subject to the above conditions as type II geometry. Supergravity models constructed with type II geometry are often referred to as $\mathcal{N}=(2,0)$ supergravity or type II supergravity. Imposing only the conditions (4.3) and keeping in mind eq. (3.5e), one can see that the residual gauge transformations are generated by the superfield $\sigma$ subject to the constraint

$$
\mathcal{D}^{2} \mathrm{e}^{-\sigma}=0
$$

\subsection{The superconformal setting}

Type II geometry can be used to describe type II supergravity and its matter couplings. However, as mentioned in previous sections, it is advantagous to make use of conformal superspace from the point of view of component reduction. The more general framework can always be reduced to conventional superspace. Below we elucidate the description of type II supergravity in this setting.

\subsubsection{The real linear compensator}

In conformal superspace, the real linear compensator $G$ is a primary nowhere vanishing scalar superfield of dimension 1 ,

$$
\nabla^{2} G=0, \quad \mathbb{D} G=G, \quad K_{A} G=0 .
$$

The constraint (4.5) allows us to express $G$ in terms of a prepotential $\mathcal{V}$ as follows:

$$
G=\mathrm{i} \nabla^{\alpha} \bar{\nabla}_{\alpha} \mathcal{V},
$$

where $\mathcal{V}$ possesses the gauge transformations

$$
\delta \mathcal{V}=\Lambda+\bar{\Lambda}, \quad \bar{\nabla}_{\alpha} \Lambda=0,
$$

with the gauge parameter $\Lambda$ being an arbitrary covariantly chiral dimensionless scalar. 
One can associate with $\mathcal{V}$ a gauge one-form $V=E^{A} V_{A}$ describing the vector multiplet. Modulo an exact one-form, we can choose the components of $V$ as follows:

$$
V_{\alpha}=\mathrm{i} \nabla_{\alpha} \mathcal{V}, \quad \bar{V}_{\alpha}=-\mathrm{i} \bar{\nabla}_{\alpha} \mathcal{V}, \quad V_{a}=-\frac{1}{2}\left(\gamma_{a}\right)^{\alpha \beta}\left[\nabla_{\alpha}, \bar{\nabla}_{\beta}\right] \mathcal{V} .
$$

The corresponding gauge-invariant field strength is simply given by $F=\mathrm{d} V$. In the complex basis the field strength is

$$
F=\bar{E}^{\beta} \wedge E^{\alpha} F_{\alpha \beta}+E^{\beta} \wedge E^{a} F_{a \beta}+\bar{E}^{\beta} \wedge E^{a} \bar{F}_{a \beta}+\frac{1}{2} E^{b} \wedge E^{a} F_{a b}
$$

where

$$
\begin{aligned}
F_{\alpha \beta} & =-2 \varepsilon_{\alpha \beta} G, \\
F_{a \beta} & =\mathrm{i}\left(\gamma_{a}\right)_{\beta}{ }^{\gamma} \nabla_{\gamma} G, \\
\bar{F}_{a \beta} & =-\mathrm{i}\left(\gamma_{a}\right)_{\beta} \bar{\nabla}_{\gamma} G, \\
F_{a b} & =\frac{1}{4} \varepsilon_{a b c}\left(\gamma^{c}\right)^{\gamma \delta}\left[\nabla_{\gamma}, \bar{\nabla}_{\delta}\right] G .
\end{aligned}
$$

One can use $G$ to construct dimensionless covariant derivatives that preserve the primary property of superfields. They are given by

$$
\begin{aligned}
& \mathscr{D}_{\alpha}=G^{-\frac{1}{2}}\left(\nabla_{\alpha}-\left(\nabla^{\beta} \ln G\right) M_{\alpha \beta}+\left(\nabla_{\alpha} \ln G\right) J-\left(\nabla_{\alpha} \ln G\right) \mathbb{D}\right), \\
& \mathscr{D}_{a}=-\frac{\mathrm{i}}{4}\left(\gamma_{a}\right)^{\alpha \beta}\left\{\mathscr{D}_{\alpha}, \overline{\mathscr{D}}_{\beta}\right\}+\mathrm{i} \mathscr{C}_{a} J+2 \mathscr{S} M_{a},
\end{aligned}
$$

where we have defined

$$
\begin{aligned}
\mathscr{S} & :=-\frac{\mathrm{i}}{4 G} \nabla^{\gamma} \bar{\nabla}_{\gamma} \ln G, \\
\mathscr{C}_{\alpha \beta} & :=-\frac{1}{4}\left[\nabla_{(\alpha}, \bar{\nabla}_{\beta)}\right] \frac{1}{G} .
\end{aligned}
$$

One can check that $G$ is covariantly constant with respect to $\mathscr{D}_{A}$. Furthermore, on primary superfields the covariant derivatives $\mathscr{D}_{A}$ satisfy the algebra

$$
\begin{aligned}
& \left\{\mathscr{D}_{\alpha}, \mathscr{D}_{\beta}\right\}=0, \\
& \left\{\mathscr{D}_{\alpha}, \overline{\mathscr{D}}_{\beta}\right\}=-2 \mathrm{i} \mathscr{D}_{\alpha \beta}-4 \mathrm{i} \varepsilon_{\alpha \beta} \mathscr{S} J+4 \mathrm{i} \mathscr{S} M_{\alpha \beta}-2 \varepsilon_{\alpha \beta} \mathscr{C}^{\gamma \delta} M_{\gamma \delta},
\end{aligned}
$$

which formally coincides with the algebra (3.3) with $\mathcal{R}=\overline{\mathcal{R}}=0$.

General supergravity invariants may be realized in the form

$$
S=\int \mathrm{d}^{3 \mid 4} z E G \mathfrak{L}\left(G, \mathscr{T}, \mathscr{D} \mathscr{T}, \mathscr{D}^{2} \mathscr{T}, \cdots\right)
$$

where $\mathfrak{L}$ is a dimensionless superfield constructed out of the torsion components $\mathscr{T}$, their covariant derivatives and the compensator $G$.

We can relate the superconformal framework to the one of conventional superspace by gauge fixing the additional symmetries. We can use the conformal boosts and $S$ supersymmetry transformations to impose the gauge condition

$$
B_{A}=0,
$$


which degauges conformal superspace to conventional superspace. The composites (4.12) become the following super-Weyl invariant objects

$$
\begin{aligned}
\mathscr{S} & :=-\frac{\mathrm{i}}{4 G}\left(\mathcal{D}^{\gamma} \overline{\mathcal{D}}_{\gamma} \ln G+4 \mathrm{i} \mathcal{S}\right), \\
\mathscr{C}_{\alpha \beta} & :=-\frac{1}{4}\left(\left[\mathcal{D}_{(\alpha}, \overline{\mathcal{D}}_{\beta)}\right]-4 \mathcal{C}_{\alpha \beta}\right) \frac{1}{G} .
\end{aligned}
$$

Upon imposing the additional gauge condition

$$
G=1
$$

the composites $\mathscr{C}_{\alpha \beta}$ and $\mathscr{S}$ can be seen to coincide with the torsion components $\mathcal{C}_{\alpha \beta}$ and $\mathcal{S}$, respectively.

It is worth mentioning that one can use the compensator to construct super-Weyl invariant covariant derivatives $\mathcal{D}_{A}^{(G)}$ in conventional superspace as follows:

$$
\begin{aligned}
& \mathcal{D}_{\alpha}^{(G)}=G^{-\frac{1}{2}}\left(\mathcal{D}_{\alpha}-\left(\mathcal{D}^{\beta} \ln G\right) M_{\alpha \beta}+\left(\mathcal{D}_{\alpha} \ln G\right) J\right), \\
& \mathcal{D}_{a}^{(G)}=-\frac{\mathrm{i}}{4}\left(\gamma_{a}\right)^{\alpha \beta}\left\{\mathcal{D}_{\alpha}^{(G)}, \overline{\mathcal{D}}_{\beta}^{(G)}\right\}+\mathrm{i} \mathscr{C}_{a} J+2 \mathscr{S} M_{a},
\end{aligned}
$$

where $\mathscr{C}_{a}$ and $\mathscr{S}$ are given by eqs. (4.16). It can be checked that these covariant derivatives satisfy the same algebra as (4.13). Unlike the operators $\mathscr{D}_{A}$, eq. (4.11), they do not annihilate the compensator $G$.

It is worth noting that $G \mathscr{S}$ turns out to be proportional to the composite linear multiplet ${ }^{14}$

$$
\mathbb{G}:=\mathrm{i} \nabla^{\gamma} \bar{\nabla}_{\gamma} \ln \left(\frac{G}{\Phi \bar{\Phi}}\right)=\mathrm{i} \nabla^{\gamma} \bar{\nabla}_{\gamma} \ln G
$$

where $\Phi$ is an arbitrary dimension $1 / 2$ chiral superfield. The composite $\mathbb{G}$ will be useful in the construction of supergravity invariants.

It should be mentioned that one can construct other composite multiplets by simply choosing the prepotential $\mathcal{V}$ of a linear multiplet to be built out of $G$. It is also possible to engineer composite linear multiplets with the use of a number of real linear multiplets $G^{\mathrm{I}} \cdot{ }^{15}$ For example, we can construct the following composite linear superfields:

$$
\mathbb{G}_{\mathcal{A}}=\mathrm{i} \nabla^{\gamma} \bar{\nabla}_{\gamma} \mathcal{A}\left(G^{\mathrm{I}}\right), \quad \mathbb{G}_{\mathcal{B}}=\mathrm{i} \nabla^{\gamma} \bar{\nabla}_{\gamma} \ln \left[\frac{\mathcal{B}\left(G^{\mathrm{I}}\right)}{\Phi \bar{\Phi}}\right],
$$

where $\mathcal{A}$ is a real homogeneous function of $G^{\mathrm{I}}$ of degree zero and $\mathcal{B}$ is a real homogeneous function of $G^{\mathrm{I}}$ of degree $1,{ }^{16}$

$$
G^{\mathrm{I}} \frac{\partial}{\partial G^{\mathrm{I}}} \mathcal{A}=0, \quad G^{\mathrm{I}} \frac{\partial}{\partial G^{\mathrm{I}}} \mathcal{B}=\mathcal{B}
$$

We will not make use of the composites $\mathbb{G}_{\mathcal{A}}$ and $\mathbb{G}_{\mathcal{B}}$ in what follows.

\footnotetext{
${ }^{14}$ This composite first appeared explicitly in conventional superspace in [18].

${ }^{15}$ Models with a number of real linear multiplets were considered in [33].

${ }^{16}$ The composite vector multiplets constructed in [23] coincide with $\mathcal{B}=C_{\mathrm{IJ}} G^{\mathrm{I}} G^{\mathrm{J}}$ with $C_{\mathrm{IJ}}$ a homogeneous function of $G^{\mathrm{I}}$ of degree $-1, G^{\mathrm{I}} \frac{\partial}{\partial G^{\mathrm{I}}} C_{\mathrm{JK}}=-C_{\mathrm{JK}}$.
} 


\subsubsection{The $B F$ action}

One can perform component reduction of superspace integrals by reducing to a chiral subspace and making use of the component reduction formula (3.27). However, many of our type II supergravity invariants can be conveniently rewritten as a $B F$ term for a composite linear multiplet. The locally supersymmetric $B F$ action can be written as

$$
S_{B F}=\int \mathrm{d}^{3 \mid 4} z E \mathcal{V} \boldsymbol{G} \text {. }
$$

Here $\mathcal{V}=\overline{\mathcal{V}}$ is the gauge prepotential of an Abelian vector multiplet, and $\boldsymbol{G}$ is a real linear superfield. The $B F$ action reduces in components to [19]

$$
\begin{aligned}
S_{B F}= & -\frac{1}{8} \int \mathrm{d}^{3} x e\left(\varepsilon^{a b c} v_{a} \boldsymbol{f}_{b c}+\frac{\mathrm{i}}{2} \lambda^{\gamma} \overline{\boldsymbol{\lambda}}_{\gamma}+\frac{\mathrm{i}}{2} \boldsymbol{\lambda}^{\gamma} \bar{\lambda}_{\gamma}+g \boldsymbol{h}+\boldsymbol{g} h\right. \\
& -\frac{1}{2}\left(\gamma^{a}\right)_{\gamma \delta} \psi_{a}^{\gamma}\left(g \boldsymbol{\lambda}^{\delta}+\boldsymbol{g} \lambda^{\delta}\right)+\frac{1}{2}\left(\gamma^{a}\right)_{\gamma \delta} \bar{\psi}_{a}^{\gamma}\left(g \overline{\boldsymbol{\lambda}}^{\delta}+\boldsymbol{g} \bar{\lambda}^{\delta}\right) \\
& \left.-\mathrm{i} \varepsilon^{a b c}\left(\gamma_{a}\right)_{\gamma \delta} \psi_{b}{ }^{\gamma} \bar{\psi}_{c}^{\delta} g \boldsymbol{g}\right),
\end{aligned}
$$

where the component fields are defined by ${ }^{17}$

$$
\begin{aligned}
g & :=G\left|, \quad \lambda_{\alpha}:=-2 \nabla_{\alpha} G\right|, \quad \bar{\lambda}_{\alpha}:=-2 \bar{\nabla}_{\alpha} G\left|, \quad h:=\mathrm{i} \bar{\nabla}^{\gamma} \nabla_{\gamma} G\right|, \\
v_{a} & :=e_{a}{ }^{m} V_{m}\left|=e_{m}{ }^{a} V_{a}\right|+\frac{1}{2} \psi_{m}{ }^{\alpha} V_{\alpha}\left|+\frac{1}{2} \bar{\psi}_{m \alpha} \bar{V}^{\alpha}\right| .
\end{aligned}
$$

The component field strength can be constructed as follows

$$
\begin{aligned}
f_{a b}:= & F_{a b}\left|-\psi_{[a}{ }^{\beta} F_{b] \beta}\right|-\bar{\psi}_{[a}{ }^{\beta} \bar{F}_{b] \beta} \mid-\frac{1}{2} \psi_{[a}{ }^{\alpha} \bar{\psi}_{b]}{ }^{\beta} F_{\alpha \beta} \\
= & \frac{1}{4} \varepsilon_{a b c}\left(\gamma^{c}\right)^{\gamma \delta}\left[\nabla_{\gamma}, \bar{\nabla}_{\delta}\right] G \mid+\frac{\mathrm{i}}{2} \psi_{[a}{ }^{\beta}\left(\gamma_{b]}\right)^{\gamma} \lambda_{\gamma} \\
& -\frac{\mathrm{i}}{2} \bar{\psi}_{[a}{ }^{\beta}\left(\gamma_{b]}\right)_{\beta}{ }^{\gamma} \bar{\lambda}_{\gamma}+\psi_{[a}{ }^{\alpha} \bar{\psi}_{b] \alpha} g .
\end{aligned}
$$

The same definitions hold for the component fields of $\boldsymbol{G}$. The component fields of the Weyl multiplet are defined as in the type I case.

To fix additional symmetries in our invariants one can make use of the gauge conditions (4.15) and (4.17), which leads to the following gauge conditions at the component level

$$
g=1, \quad \lambda_{\alpha}=0, \quad b_{m}=0 .
$$

The first fixes the dilatations, the second fixes the $S$-supersymmetry transformations and the last fixes the special conformal boosts. To see what the invariants we construct correspond to at the component level we give the following useful results in the gauge (4.25) (compare with (2.27)):

$$
\begin{aligned}
\square g & =\frac{1}{4} R+\text { fermion terms } \\
\square^{2} g & =R^{a b} R_{a b}-\frac{1}{8} R^{2}+\frac{1}{4} D^{a} D_{a} R+\text { fermion terms }
\end{aligned}
$$

\footnotetext{
${ }^{17}$ The supersymmetry transformations of the component fields were given in [19].
} 
where we have defined

$$
\begin{aligned}
\square g & :=\nabla^{a} \nabla_{a} G \mid, \\
\square^{2} g & :=\nabla^{a} \nabla_{a} \nabla^{b} \nabla_{b} G \mid .
\end{aligned}
$$

\subsection{Supergravity invariants}

We will write down actions for various supergravity models by constructing a superspace Lagrangian built out of $G$, the composites (4.12a) and (4.12b), and their $\mathscr{D}$-covariant derivatives.

\subsubsection{The supergravity action}

The type II minimal supergravity action with a cosmological term was given in [18] in conventional superspace. In conformal superspace it is given by

$$
S=\frac{1}{\kappa} S_{\mathrm{SG}}+\lambda S_{\mathrm{cos}}
$$

where

$$
\begin{aligned}
& S_{\mathrm{SG}}=4 \int \mathrm{d}^{3 \mid 4} z E G \ln \left(\frac{G}{\Phi \bar{\Phi}}\right), \\
& S_{\mathrm{cos}}=4 \int \mathrm{d}^{3 \mid 4} z E \mathcal{V} G
\end{aligned}
$$

and $\lambda$ is the cosmological constant. Integrating by parts leads to the following equivalent form for $S_{\mathrm{SG}}$ :

$$
S_{\mathrm{SG}}=4 \int \mathrm{d}^{3 \mid 4} z E \mathcal{V} \mathbb{G}=-16 \int \mathrm{d}^{3 \mid 4} z E \mathcal{V} G \mathscr{S} .
$$

The corresponding component action may be derived by putting $\boldsymbol{G} \rightarrow \mathbb{G}$ into the $B F$ action (4.23). It is straighforward to show that the component field $\boldsymbol{h}$ contains a term proportional to $\frac{1}{g} \square g$. Making use of the gauge conditions (4.25) and the results (4.26), one can see that it gives rise to the Einstein-Hilbert term in the component action. The cosmological term comes from the U(1) Chern-Simons term described by the invariant $S_{\text {cos }}$. The full component action for supergravity with a cosmological term was analysed in detail in [18].

\subsubsection{The $R h^{n}$ invariants}

One can construct an invariant containing a $R h^{n}$ term with $n>1$ as follows

$$
\begin{aligned}
S_{R h^{n}} & =\int \mathrm{d}^{3 \mid 4} z E\left(\frac{\mathbb{G}}{G}\right)^{n} G=\int \mathrm{d}^{3 \mid 4} z E\left(\frac{\mathbb{G}}{G}\right)^{n-1} \mathbb{G} \\
& =(-4)^{n} \int \mathrm{d}^{3 \mid 4} z E G \mathscr{S}^{n} .
\end{aligned}
$$

Upon integrating by parts one finds the equivalent forms

$$
S_{R h^{n}}=\int \mathrm{d}^{3 \mid 4} z E \mathcal{V} \mathbb{G}_{n}=\int \mathrm{d}^{3 \mid 4} z E \mathbb{G}_{n-1} \ln \left(\frac{G}{\Phi \bar{\Phi}}\right),
$$


where

$$
\mathbb{G}_{n}=\mathrm{i} \nabla^{\gamma} \bar{\nabla}_{\gamma}\left(\frac{\mathbb{G}}{G}\right)^{n}
$$

The component form of the above action can be obtained from the $B F$ action (4.23) by putting for instance

$$
G \rightarrow \mathbb{G}_{n}
$$

into the $B F$ action (4.23). It is straightforward to check that the component action contains the term

$$
\frac{3 n(n-1)}{16} R h^{n}
$$

upon imposing the gauge conditions (4.25).

For $n=1$ the invariant (4.31) vanishes and so we have to consider the $n=1$ case separately. A locally supersymmetric invariant containing a $R h$ term is described by

$$
S_{R h}=-4 \int \mathrm{d}^{3 \mid 4} z E \mathbb{G} \ln \left(\frac{G}{\Phi \bar{\Phi}}\right)=16 \int \mathrm{d}^{3 \mid 4} z E G \mathscr{S} \ln \left(\frac{G}{\Phi \bar{\Phi}}\right) .
$$

The component action can be worked out by putting $G \rightarrow \mathbb{G}$ and $G \rightarrow \mathbb{G}$ into the $B F$ action (4.23). It gives rise to a term proportional to $\frac{1}{g^{2}} h \square g$, which upon gauge fixing leads to the $R h$ term in the component action. The bosonic action was explictly given in [23].

It is important to note that in the $n=2$ case the action also contains a scalar curvature squared term. However, as was discussed in [23], an independent invariant containing a curvature squared term is not known to exist. This can be attributed to the fact that only a real scalar composite $\mathscr{S}$ can be constructed from the linear multiplet, while for the type I case one can construct a complex scalar composite $\mathscr{R}$ leading to an extra invariant.

\subsubsection{The Ricci curvature squared invariant}

An invariant containing a Ricci squared term is given by

$$
S_{\mathrm{Ricci}^{2}}=4 \int \mathrm{d}^{3 \mid 4} z E G \mathscr{C}^{\alpha \beta} \mathscr{C}_{\alpha \beta}
$$

The action also contains a scalar curvature squared term contribution. This can be checked by using the results of [18]. It is natural to introduce the one parameter family of invariants

$$
S_{\zeta}=4 \int \mathrm{d}^{3 \mid 4} z E G\left(\mathscr{C}^{\alpha \beta} \mathscr{C}_{\alpha \beta}+\zeta \mathscr{S}^{2}\right)
$$

where $\zeta$ parametrizes the scalar curvature squared contribution.

The invariant $S_{\mathrm{Ricci}^{2}}=S_{0}$ can be seen to correspond to the one given in [23] with the gauge conditions (4.15) and (4.17). In this gauge we find $\mathcal{R}=0$ and $\mathcal{C}_{\alpha \beta}$ satisfies a constraint reminiscent of a $\mathcal{N}=2$ Yang-Mills multiplet

$$
\mathcal{D}^{2} \mathcal{C}_{\alpha \beta}=0
$$


The above constraints mean that the supersymmetry transformations of $\mathcal{C}_{\alpha \beta}$ can be put into one-to-one correspondence with a Yang-Mills multiplet. The action with the gauge conditions (4.15) and (4.17) reads

$$
S_{\text {Ricii }^{2}}=4 \int \mathrm{d}^{3 \mid 4} z E \mathcal{C}^{\alpha \beta} \mathcal{C}_{\alpha \beta}
$$

However, we can identity this action up to some multiplicative constant as a special case of the Yang-Mills action

$$
\int \mathrm{d}^{3 \mid 4} z E \operatorname{tr} \mathcal{G}^{2}
$$

where $\mathcal{G}=\mathcal{C}^{\alpha \beta} M_{\alpha \beta}$ and we are tracing over the Lorentz group. Therefore we can equally construct the action in the gauge $G=1$ using the correspondence with the Yang-Mills multiplet. This provides a geometric explanation for the procedure employed in [23] at the component level. However, in our approach it is not necessary to work in the gauge (4.25) since one can just use the action (4.37).

Finally, It is worth mentioning that a pure Ricci curvature squared invariant is given by

$$
S_{\mathrm{Ricci}^{2}}^{(\text {pure }}=S_{-40 / 3}=4 \int \mathrm{d}^{3 \mid 4} z E G\left(\mathscr{C}^{\alpha \beta} \mathscr{C}_{\alpha \beta}-\frac{40}{3} \mathscr{S}^{2}\right) .
$$

In the gauge (4.25) it gives rise to a $R^{a b} R_{a b}$ term in the component action.

It should be mentioned that although we have restricted our attention to curvature squared terms. Similarly to the type I case, one can always consider higher derivative and locally supersymmetric actions by considering other instances of the action (4.14). One should mention the following important descendent of the primary composites (4.12):

$$
\mathscr{W}_{\alpha \beta}=-\frac{\mathrm{i}}{4}\left[\mathscr{D}^{\gamma}, \overline{\mathscr{D}}_{\gamma}\right] \mathscr{C}_{\alpha \beta}+\frac{1}{2}\left[\mathscr{D}_{(\alpha}, \overline{\mathscr{D}}_{\beta)}\right] \mathscr{S}+2 \mathscr{S} \mathscr{C}_{\alpha \beta}
$$

It is related to the super-Cotton tensor $W_{\alpha \beta}$ as

$$
W_{\alpha \beta}=G^{2} \mathscr{W}_{\alpha \beta}
$$

It should be kept in mind that the super-Cotton tensor is actually independent of the compensator $G$.

\subsection{Models for massive supergravity}

In this section, in analogy to type I supergravity, we analyse the equations of motion for a general supergravity model and determine the conditions in which we have massive theories of supergravity.

We consider the following type II supergravity model

$$
S=\lambda S_{\mathrm{cos}}+\frac{1}{\kappa} S_{\mathrm{SG}}+\frac{1}{\tilde{\mu}} S_{\mathrm{CSG}}+\mu_{1} S_{R h}+\mu_{2} S_{R h^{2}}+\mu_{3} S_{\mathrm{Ricci}^{2}},
$$

where $\kappa, \tilde{\mu}, \mu_{1}, \mu_{2}$ and $\mu_{3}$ are real, and we make use of the invariants defined in subsection 4.3. Here $S_{\mathrm{CSG}}$ denotes the $\mathcal{N}=2$ conformal supergravity action given in our conventions in [31]. Here we assume $\mu_{3} \geq 0$ as in [23]. 
The equations of motion corresponding to the theory with action (4.45) can be derived by varying the action with respect to the prepotential $\mathcal{V}$ of the real linear compensator $G$. One finds the equation of motion on the compensator to be

$$
\begin{aligned}
0= & \mathrm{i} \mathscr{D}^{\alpha} \overline{\mathscr{D}}_{\alpha}\left[8 \mu_{1} \mathscr{S}-2 \mu_{2}\left(\mathrm{i} \mathscr{D}^{\beta} \overline{\mathscr{D}}_{\beta} \mathscr{S}+2 \mathrm{i} \mathscr{S}^{2}\right)+\mu_{3}\left(2 \mathrm{i} \mathscr{D}^{\beta} \overline{\mathscr{D}}_{\beta} \mathscr{S}-\mathscr{C}^{\alpha \beta} \mathscr{C}_{\alpha \beta}\right)\right] \\
& +2 \lambda-\frac{4}{\kappa} \mathscr{S} .
\end{aligned}
$$

The equation of motion for the conformal supergravity prepotential is

$$
\frac{1}{\mu} W_{\alpha \beta}+T_{\alpha \beta}=0
$$

where $\mu$ is related to $\tilde{\mu}$ by a multiplicative constant. One can find the supergravity equation of motion (4.47) by making use of the results for the deformation of the prepotential in appendix $\mathrm{C}$, which imply

$$
\delta S_{\mathrm{CSG}}=\int \mathrm{d}^{3 \mid 4} z E \delta H^{\alpha \beta} W_{\alpha \beta}, \quad \delta\left[S-\frac{1}{\tilde{\mu}} S_{\mathrm{CSG}}\right]=\int \mathrm{d}^{3 \mid 4} z E \delta H^{\alpha \beta} T_{\alpha \beta} .
$$

It can be checked that the supercurrent $T_{\alpha \beta}$ is given by

$$
T_{\alpha \beta}=G^{2} \mathbb{T}_{\alpha \beta},
$$

where

$$
\mathbb{T}_{\alpha \beta}=\lambda \mathbb{T}^{(\cos )}{ }_{\alpha \beta}+\frac{1}{\kappa} \mathbb{T}_{\alpha \beta}^{(\mathrm{SG})}+\mu_{1} \mathbb{T}_{\alpha \beta}^{(\mathrm{R} h)}+\mu_{2} \mathbb{T}^{\left(\mathrm{R} h^{2}\right)}{ }_{\alpha \beta}+\mu_{3} \mathbb{T}^{\left(\mathrm{Ricci}^{2}\right)}{ }_{\alpha \beta},
$$

and

$$
\begin{aligned}
& \mathbb{T}^{(\cos )}{ }_{\alpha \beta}=2\left[\mathscr{D}_{(\alpha}, \overline{\mathscr{D}}_{\beta)}\right] \mathcal{V},
\end{aligned}
$$

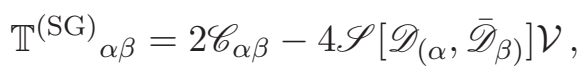

$$
\begin{aligned}
& \mathbb{T}^{(\mathcal{R} h)}{ }_{\alpha \beta}=4\left(\left[\mathscr{D}_{(\alpha}, \overline{\mathscr{D}}_{\beta)}\right]+4 \mathscr{C}_{\alpha \beta}\right) \mathscr{S}+8\left(\left[\mathscr{D}_{(\alpha}, \overline{\mathscr{D}}_{\beta)}\right] \mathcal{V}\right) \mathrm{i} \mathscr{D}^{\gamma} \overline{\mathscr{D}}_{\gamma} \mathscr{S}, \\
& \mathbb{T}^{\left(\mathcal{R} h^{n}\right)_{\alpha \beta}}=-(-4)^{n}\left\{\frac{n}{32}\left(\left[\mathscr{D}_{(\alpha}, \overline{\mathscr{D}}_{\beta}\right]+4 \mathscr{C}_{\alpha \beta}\right) \mathrm{i} \mathscr{D}^{\gamma} \overline{\mathscr{D}}_{\gamma} \mathscr{S}^{(n-1)}\right. \\
& \left.\left.+\frac{n(n-1)}{2} \mathscr{S}^{n-2}\left(\mathscr{D}_{(\alpha} \mathscr{S}\right) \overline{\mathscr{D}}_{\beta}\right) \mathscr{S}\right\} \\
& -\frac{(-4)^{n}}{16}\left(\left[\mathscr{D}_{(\alpha}, \overline{\mathscr{D}}_{\beta)}\right] \mathcal{V}\right) \mathrm{i} \mathscr{D}^{\gamma} \overline{\mathscr{D}}_{\gamma}\left(n \mathrm{i} \mathscr{D}^{\gamma} \overline{\mathscr{D}}_{\gamma} \mathscr{S}^{(n-1)}+4(n-1) \mathscr{S}^{n}\right), \\
& \mathbb{T}^{\left(\mathrm{Ricci}^{2}{ }_{\alpha \beta}\right.}=-\frac{1}{4}\left[\mathscr{D}^{\gamma}, \overline{\mathscr{D}}_{\gamma}\right]\left[\mathscr{D}^{\delta}, \overline{\mathscr{D}}_{\delta}\right] \mathscr{C}_{\alpha \beta}-4 \mathscr{D}^{a} \mathscr{D}_{a} \mathscr{C}_{\alpha \beta}+\mathrm{i} \mathscr{D}_{(\alpha}{ }^{\gamma}\left[\mathscr{D}^{\delta}, \overline{\mathscr{D}}_{|\delta|}\right] \mathscr{C}_{\beta) \gamma} \\
& +24 \overline{\mathscr{C}}_{\alpha \beta \gamma} \mathscr{D}^{\gamma} \mathscr{S}-24 \mathscr{C}_{\alpha \beta \gamma} \overline{\mathscr{D}}^{\gamma} \mathscr{S}+\left[\mathscr{D}_{(\alpha}, \overline{\mathscr{D}}_{\beta)}\right] \mathscr{C}^{\gamma \delta} \mathscr{C}_{\gamma \delta} \\
& -\frac{16}{3} \mathscr{C}_{\alpha \beta} \mathrm{i} \mathscr{D}^{\gamma} \overline{\mathscr{D}}_{\gamma} \mathscr{S}+4 \mathscr{C}^{\gamma \delta}\left(\mathrm{i} \overline{\mathscr{D}}_{(\alpha} \mathscr{C}_{\beta \gamma \delta)}+\mathrm{i} \mathscr{D}_{(\alpha} \overline{\mathscr{C}}_{\beta \gamma \delta)}\right)+8 \mathscr{C}_{(\alpha}{ }^{\gamma} \mathscr{D}_{\beta) \gamma} \mathscr{S} \\
& -4 \mathrm{i} \mathscr{S}\left[\mathscr{D}^{\gamma}, \overline{\mathscr{D}}_{\gamma}\right] \mathscr{C}_{\alpha \beta}-8 \mathscr{S} \varepsilon^{c a b}\left(\gamma_{c}\right)_{\alpha \beta} \mathscr{D}_{a} \mathscr{C}_{b}-32 \mathscr{S}^{2} \mathscr{C}_{\alpha \beta} \\
& +\left(\left[\mathscr{D}_{(\alpha}, \overline{\mathscr{D}}_{\beta)}\right] \mathcal{V}\right) \mathrm{i} \mathscr{D}^{\gamma} \overline{\mathscr{D}}_{\gamma}\left(2 \mathrm{i} \mathscr{D}^{\gamma} \overline{\mathscr{D}}_{\gamma} \mathscr{S}-\mathscr{C}_{\alpha \beta} \mathscr{C}^{\alpha \beta}\right) \text {. }
\end{aligned}
$$


Making use of the compensator equation of motion, the above expression becomes

$$
\begin{aligned}
& 0=\frac{1}{\mu} \mathscr{W}_{\alpha \beta}+\frac{2}{\kappa} \mathscr{C}_{\alpha \beta}+4 \mu_{1}\left(\left[\mathscr{D}_{(\alpha}, \overline{\mathscr{D}}_{\beta)}\right]+4 \mathscr{C}_{\alpha \beta}\right) \mathscr{S} \\
& -\mu_{2}\left[\left(\left[\mathscr{D}_{(\alpha}, \overline{\mathscr{D}}_{\beta)}\right]+4 \mathscr{C}_{\alpha \beta}\right) \mathrm{i} \mathscr{D}^{\gamma} \overline{\mathscr{D}}_{\gamma} \mathscr{S}+16\left(\mathscr{D}_{(\alpha} \mathscr{S}\right) \overline{\mathscr{D}}_{\beta)} \mathscr{S}\right] \\
& -\frac{\mu_{3}}{4}\left[\left[\mathscr{D}^{\gamma}, \overline{\mathscr{D}}_{\gamma}\right]\left[\mathscr{D}^{\delta}, \overline{\mathscr{D}}_{\delta}\right] \mathscr{C}_{\alpha \beta}+16 \mathscr{D}^{a} \mathscr{D}_{a} \mathscr{C}_{\alpha \beta}-4 \mathrm{i} \mathscr{D}_{(\alpha}{ }^{\gamma}\left[\mathscr{D}^{\delta}, \overline{\mathscr{D}}_{|\delta|}\right] \mathscr{C}_{\beta) \gamma}\right. \\
& -96 \overline{\mathscr{C}}_{\alpha \beta \gamma} \mathscr{D}^{\gamma} \mathscr{S}+96 \mathscr{C}_{\alpha \beta \gamma} \overline{\mathscr{D}}^{\gamma} \mathscr{S}-4\left[\mathscr{D}_{(\alpha}, \overline{\mathscr{D}}_{\beta)}\right] \mathscr{C}^{\gamma \delta} \mathscr{C}_{\gamma \delta} \\
& +\frac{64}{3} \mathscr{C}_{\alpha \beta} \mathrm{i} \mathscr{D}^{\gamma} \overline{\mathscr{D}}_{\gamma} \mathscr{S}-16 \mathscr{C}^{\gamma \delta}\left(\mathrm{i} \overline{\mathscr{D}}_{(\alpha} \mathscr{C}_{\beta \gamma \delta)}+\mathrm{i} \mathscr{D}_{(\alpha} \overline{\mathscr{C}}_{\beta \gamma \delta)}\right)-32 \mathscr{C}_{(\alpha}{ }^{\gamma} \mathscr{D}_{\beta) \gamma} \mathscr{S} \\
& \left.+16 \mathrm{i} \mathscr{S}\left[\mathscr{D}^{\gamma}, \overline{\mathscr{D}}_{\gamma}\right] \mathscr{C}_{\alpha \beta}+32 \mathscr{S} \varepsilon^{c a b}\left(\gamma_{c}\right)_{\alpha \beta} \mathscr{D}_{a} \mathscr{C}_{b}+128 \mathscr{S}^{2} \mathscr{C}_{\alpha \beta}\right] \text {. }
\end{aligned}
$$

The supercurrent $T_{\alpha \beta}$ obeys the conservation equation

$$
\nabla^{\beta} T_{\alpha \beta}=0
$$

when the compensator obeys its equation of motion (4.46).

We are interested in maximally supersymmetric solutions of the supergravity equations of motion. In type II supergravity backgrounds, all maximally supersymmetric backgrounds $[18,65]$ are characterised by dimension- 1 torsion superfields under the following constraints

$$
\mathcal{R}=0, \quad \mathcal{D}_{A} \mathcal{S}=0, \quad \mathcal{D}_{\alpha} \mathcal{C}_{b}=0 \quad \Longrightarrow \quad \mathcal{D}_{a} \mathcal{C}_{b}=2 \varepsilon_{a b c} \mathcal{C}^{c} \mathcal{S}, \quad \mathcal{C}^{a} \mathcal{C}_{a}=\text { const }
$$

The corresponding algebra of covariant derivatives is

$$
\begin{aligned}
\left\{\mathcal{D}_{\alpha}, \mathcal{D}_{\beta}\right\} & =0, \\
\left\{\mathcal{D}_{\alpha}, \overline{\mathcal{D}}_{\beta}\right\} & =-2 \mathrm{i}\left(\gamma^{c}\right)_{\alpha \beta}\left(\mathcal{D}_{c}-2 \mathcal{S} M_{c}-\mathrm{i} \mathcal{C}_{c} J\right)+4 \varepsilon_{\alpha \beta}\left(\mathcal{C}^{c} M_{c}-\mathrm{i} \mathcal{S} J\right), \\
{\left[\mathcal{D}_{a}, \mathcal{D}_{\beta}\right] } & =\mathrm{i} \varepsilon_{a b c}\left(\gamma^{b}\right)_{\beta}{ }^{\gamma} \mathcal{C}^{c} \mathcal{D}_{\gamma}+\left(\gamma_{a}\right)_{\beta}{ }^{\gamma} \mathcal{S} \mathcal{D}_{\gamma} \\
{\left[\mathcal{D}_{a}, \mathcal{D}_{b}\right] } & =4 \varepsilon_{a b c}\left(\mathcal{C}^{c} \mathcal{C}_{d}+\delta_{d}^{c} \mathcal{S}^{2}\right) M^{d}
\end{aligned}
$$

The equations of motion (4.46) and (4.52) simplify significantly for maximally supersymmetric backgrounds where we have the conditions

$$
\mathcal{C}_{a}=0, \quad \mathcal{S}=\mathcal{S}_{0}=\text { const }
$$

In this case the equation on the compensator reduces to

$$
\mathcal{S}_{0}=\frac{\kappa}{2} \lambda
$$

and the supercurrent vanishes,

$$
T_{\alpha \beta}=W_{\alpha \beta}=0 .
$$

The supergravity equations of motion are by construction super-Weyl invariant. One can fix this super-Weyl invariance by imposing the gauge

$$
G=1 .
$$


Keep in mind that the super-Weyl invariance can be restored by replacing $\mathcal{D}_{\alpha} \rightarrow \mathscr{D}_{\alpha}, \mathcal{C}_{a} \rightarrow$ $\mathscr{C}_{a}$ and $\mathcal{S} \rightarrow \mathscr{S}$ everywhere. We will assume the above gauge condition in what follows.

We consider perturbations in the model (4.45) about the maximally supersymmetric solution:

$$
\mathcal{S}=\mathcal{S}_{0}+\Delta \mathcal{S}, \quad \mathcal{C}_{\alpha \beta}=\Delta \mathcal{C}_{\alpha \beta}
$$

The equation of motion on the compensator becomes

$$
0=\frac{2}{\kappa} \Delta \mathcal{S}-4\left(\mu_{1}-\mu_{2} \mathcal{S}_{0}\right) \mathrm{i} \mathcal{D}^{\alpha} \overline{\mathcal{D}}_{\alpha} \Delta \mathcal{S}+\left(\mu_{2}-\mu_{3}\right)\left(\mathrm{i} \mathcal{D}^{\alpha} \overline{\mathcal{D}}_{\alpha}\right)^{2} \Delta \mathcal{S} .
$$

Thus we see that $\Delta \mathcal{S}$ is propagating in general. However one can simplify the equation of motion (4.61) by turning it into an algebraic one by setting

$$
\mu_{1}=\mu_{2} \mathcal{S}_{0}, \quad \mu_{3}=\mu_{2} .
$$

In this case, if $\frac{1}{\kappa} \neq 0$ we must require $\Delta \mathcal{S}=0$.

By linearizing the Bianchi identity (3.4) about the background chosen one can show that $\Delta \mathcal{C}_{\alpha \beta}$ is divergenceless, $\mathcal{D}^{\beta} \Delta \mathcal{C}_{\alpha \beta}=0$. Using this condition one can write the supergravity equation of motion in the form

$$
\begin{aligned}
0= & \mu_{3}\left(\mathrm{i} \mathcal{D}^{\gamma} \overline{\mathcal{D}}_{\gamma}\right)^{2} \Delta \mathcal{C}_{\alpha \beta}-\left(8 \mu_{3} \mathcal{S}_{0}+\frac{1}{2 \mu}\right) \mathrm{i} \mathcal{D}^{\gamma} \overline{\mathcal{D}}_{\gamma} \Delta \mathcal{C}_{\alpha \beta} \\
& +2\left(\frac{1}{\kappa}+\frac{1}{\mu} \mathcal{S}_{0}-32 \mu_{3} \mathcal{S}_{0}^{2}\right) \Delta \mathcal{C}_{\alpha \beta}
\end{aligned}
$$

Consistency of the previous equation may be checked by making use of a general property of symmetric divergenceless superfields in the background chosen. Specifically, given a symmetric real spinor $T_{\alpha_{1} \cdots \alpha_{n}}=T_{\left(\alpha_{1} \cdots \alpha_{n}\right)}$ such that $\mathcal{D}^{\alpha} T_{\alpha \alpha_{1} \cdots \alpha_{n-1}}=0$, one can check that the following holds

$$
\mathcal{D}^{\alpha} \mathcal{D}^{\gamma} \overline{\mathcal{D}}_{\gamma} T_{\alpha \alpha_{1} \cdots \alpha_{n-1}}=0
$$

This implies that the operator $\frac{\mathrm{i}}{2} \mathcal{D}^{\gamma} \overline{\mathcal{D}}_{\gamma}$ preserves the divergenceless condition of the superfield $\Delta \mathcal{C}_{\alpha \beta}$. Thus one can check that (4.63) is consistent.

It should be mentioned that (4.63) can also be rewritten in terms of vector covariant derivatives as follows

$$
\begin{aligned}
0= & \mu_{3} \mathcal{D}^{a} \mathcal{D}_{a} \Delta \mathcal{C}_{\alpha \beta}+\left(4 \mu_{3} \mathcal{S}_{0}-\frac{1}{4 \mu}\right) \varepsilon^{c a b}\left(\gamma_{c}\right)_{\alpha \beta} \mathcal{D}_{a} \Delta \mathcal{C}_{b} \\
& +\frac{1}{2}\left(\frac{1}{\kappa}-\frac{1}{\mu} \mathcal{S}_{0}-16 \mu_{3} \mathcal{S}_{0}^{2}\right) \Delta \mathcal{C}_{\alpha \beta} .
\end{aligned}
$$

In the case $\mu_{3}>0$ we may factorize (4.63) as follows

$$
\left(\frac{\mathrm{i}}{2} \mathcal{D}^{\gamma} \overline{\mathcal{D}}_{\gamma}+m_{-}\right)\left(\frac{\mathrm{i}}{2} \mathcal{D}^{\gamma} \overline{\mathcal{D}}_{\gamma}-m_{+}\right) \Delta \mathcal{C}_{\alpha \beta}=0,
$$


where

$$
\begin{aligned}
m_{+}-m_{-} & =4 \mathcal{S}_{0}+\frac{1}{4 \mu_{3} \mu}, \\
m_{+} m_{-} & =-\frac{1}{2}\left(\frac{1}{\kappa \mu_{3}}+\frac{1}{\mu \mu_{3}} \mathcal{S}_{0}-32 \mathcal{S}_{0}^{2}\right) .
\end{aligned}
$$

The constants $m_{+}$and $m_{-}$are real for

$$
\left(4 \mu_{3} \mathcal{S}_{0}+\frac{1}{4 \mu}\right)^{2} \geq 2 \mu_{3}\left(\frac{1}{\kappa}+\frac{1}{\mu} \mathcal{S}_{0}-32 \mu_{3} \mathcal{S}_{0}^{2}\right)
$$

Massive supergravity may be described by the model (4.45) with various choices of parameters. We see from eq. (4.68) that in a Minkowski background $\left(\mathcal{S}_{0}=0\right)$ with $\mu_{3}>0$ and $\mu=\infty$ it is necessary to have a negative Einstein Hilbert term, $\kappa<0$ or no EinsteinHilbert term $\kappa \rightarrow \infty$. About a non-Minkowski background, $\mathcal{S}_{0} \neq 0$, the presence of a $R h^{2}$ in the action is problematic for ghost freedom [23]. The choice $\lambda=0$ and $\mu_{1}=0$ recovers the $\mathcal{N}=2$ generalised massive supergravity model discussed in [23].

It is worth noting that maximally supersymmetric backgrounds are characterised by the more general conditions (4.54). In this case

$$
\mathcal{S}=\mathcal{S}_{0}=\text { const }, \quad \mathcal{C}^{a}=\mathcal{C}_{0}^{a}=\text { const }
$$

and the equation on the compensator reduces to

$$
\mathcal{S}_{0}=\frac{\kappa}{2} \lambda
$$

while the equation for the gravitational superfield becomes

$$
0=\left[\frac{1}{\kappa}+\left(\frac{1}{\mu}+8 \mu_{1}\right) \mathcal{S}_{0}-16 \mu_{3} \mathcal{S}_{0}^{3}\right] \mathcal{C}_{0}^{a} .
$$

We have already studied the case $\mathcal{C}_{0}^{a}=0$. If $\mathcal{C}_{0}^{a} \neq 0$ we have the condition

$$
\frac{1}{\kappa}+\left(\frac{1}{\mu}+8 \mu_{1}\right) \mathcal{S}_{0}-16 \mu_{3} \mathcal{S}_{0}^{3}=0 \text {. }
$$

We do not discuss linearization about this background here.

\section{$5 \mathcal{N}=2$ supergravity models with a complex linear compensator}

In the previous sections we have constructed $\mathcal{N}=2$ supergravity models by using a chiral and a real linear compensator. In complete analogy to four-dimensional $\mathcal{N}=1$ supergravity, see $[26,45,46]$ for detailed reviews, $3 \mathrm{D} \mathcal{N}=2$ off-shell Poincaré and AdS supergravities can be realised by using a complex linear superfield coupled to conformal supergravity. These non-minimal 3D $\mathcal{N}=2$ models were introduced in [29, 33]. In this section we aim to show that massive supergravity can be constructed in the non-minimal case. 


\subsection{Non-minimal supergravity in conventional superspace}

To describe non-minimal supergravity one makes use of a complex linear compensator $\Sigma$ that obeys the constraint

$$
\left(\overline{\mathcal{D}}^{2}-4 \mathcal{R}\right) \Sigma=0
$$

and is subject to no reality condition. By definition, the compensator $\Sigma$ is chosen to be nowhere vanishing and transform as a primary field of weight $w$ under the super-Weyl group. The $\mathrm{U}(1)_{R}$ charge of $\Sigma$ is uniquely determined [29],

$$
\delta_{\sigma} \Sigma=w \sigma \Sigma \quad \Longrightarrow \quad J \Sigma=(1-w) \Sigma .
$$

For every value of $w \neq 0,1$ the following action

$$
S_{\text {non-minimal }}=\frac{4 w}{1-w} \int \mathrm{d}^{3 \mid 4} z E(\bar{\Sigma} \Sigma)^{\frac{1}{2 w}}
$$

describes off-shell non-minimal Poincaré supergravity providing a supersymmetric extension of the Einstein-Hilbert term. On the other hand, it turns out that the complex linear superfield $\Sigma$ is not suitable to construct a cosmological constant term and describe AdS supergravity. The way around this limitation was found in the four-dimensional case in [51] and applied to three dimensions in [33]. The core of the idea is that when $w=-1$ the complex linear constraint (5.1) admits non-trivial deformations.

Consider a new conformal compensator $\Gamma$ that has the transformation properties

$$
\delta_{\sigma} \Gamma=-\sigma \Gamma, \quad J \Gamma=2 \Gamma
$$

and obeys the improved linear constraint [33]

$$
-\frac{1}{4}\left(\overline{\mathcal{D}}^{2}-4 \mathcal{R}\right) \Gamma=\lambda=\text { const } .
$$

This constraint is super-Weyl invariant and the complex parameter $\lambda \neq 0$ turns out to play the role of a cosmological constant. In fact, the action

$$
S_{\text {AdS }}=-2 \int \mathrm{d}^{3 \mid 4} z E(\bar{\Gamma} \Gamma)^{-1 / 2}
$$

describes AdS supergravity. We can prove this statement by showing that the action (5.6) is dual to the type I minimal supergravity action (3.36). Consider the first-order action

$$
S_{\text {first-order }}=\int \mathrm{d}^{3 \mid 4} z E\left(-4 \bar{\Phi} \Phi+\Gamma \Phi^{4}+\bar{\Gamma} \bar{\Phi}^{4}\right)
$$

where $\Phi$ is complex unconstrained, and $\Gamma$ obeys the constraint (5.5). Varying $S_{\text {first-order }}$ with respect to $\Gamma$ yields $\overline{\mathcal{D}}_{\alpha} \Phi=0$, and then (5.7) reduces to the supergravity matter action (3.36) where for simplicity we have set $\kappa=1$. On the other hand, we can integrate out the fields $\Phi$ and $\bar{\Phi}$ to end up with the action (5.6). In the following we will focus only on non-minimal supergravity where the compensator satisfies (5.5). 
Let us now discuss some geometrical properties of the $w=-1$ non-minimal supergravity within conventional superspace. The super-Weyl and local $\mathrm{U}(1)_{R}$ symmetries can be used to impose the gauge condition

$$
\Gamma=1
$$

In this gauge, some restrictions on the geometry occur [29]. To describe them, it is useful to split the covariant derivatives as

$$
\mathcal{D}_{\alpha}=\mathrm{D}_{\alpha}+\mathrm{i} T_{\alpha} J, \quad \overline{\mathcal{D}}_{\alpha}=\overline{\mathrm{D}}_{\alpha}+\mathrm{i} \bar{T}_{\alpha} J,
$$

where $T_{\alpha}$ is related to the original complex $\mathrm{U}(1)_{R}$ connection $\Phi_{\alpha}$ as $T_{\alpha}=-\Phi_{\alpha}$. In the gauge (5.8), the constraint $\left(\overline{\mathcal{D}}^{2}-4 \mathcal{R}\right) \Gamma=-4 \lambda$ turns into

$$
\mathcal{R}=\lambda+\frac{\mathrm{i}}{2}\left(\overline{\mathrm{D}}_{\alpha} \bar{T}^{\alpha}+\mathrm{i} \bar{T}_{\alpha} \bar{T}^{\alpha}\right)
$$

Evaluating explicitly $\left\{\mathcal{D}_{\alpha}, \mathcal{D}_{\beta}\right\} \Gamma$ and $\left\{\mathcal{D}_{\alpha}, \overline{\mathcal{D}}_{\beta}\right\} \Gamma$ and then setting $\Gamma=1$ gives

$$
\begin{aligned}
\mathrm{D}_{(\alpha} T_{\beta)} & =0, \quad \mathcal{S}=\frac{1}{8}\left(\overline{\mathrm{D}}^{\alpha} T_{\alpha}-\mathrm{D}^{\alpha} \bar{T}_{\alpha}+2 \mathrm{i} T^{\alpha} \bar{T}_{\alpha}\right), \\
\Phi_{\alpha \beta} & =\mathcal{C}_{\alpha \beta}+\frac{\mathrm{i}}{2} \mathrm{D}_{(\alpha} \bar{T}_{\beta)}+\frac{\mathrm{i}}{2} \overline{\mathrm{D}}_{(\alpha} T_{\beta)}+T_{(\alpha} \bar{T}_{\beta)} .
\end{aligned}
$$

If we define a new vector covariant derivative $\mathrm{D}_{a}$ by $\mathrm{D}_{a}:=\mathcal{D}_{a}-\mathrm{i} \Phi_{a} J$, then the algebra of the covariant derivatives $\mathrm{D}_{A}=\left(\mathrm{D}_{a}, \mathrm{D}_{\alpha}, \overline{\mathrm{D}}^{\alpha}\right)$ proves to be

$$
\begin{aligned}
\left\{\mathrm{D}_{\alpha}, \mathrm{D}_{\beta}\right\}= & -2 \mathrm{i} T_{(\alpha} \mathrm{D}_{\beta)}-4 \overline{\mathcal{R}} M_{\alpha \beta}, \\
\left\{\mathrm{D}_{\alpha}, \overline{\mathrm{D}}_{\beta}\right\}= & -2 \mathrm{iD}_{\alpha \beta}-\mathrm{i} \bar{T}_{(\alpha} \mathrm{D}_{\beta)}+\mathrm{i} T_{(\alpha} \overline{\mathrm{D}}_{\beta)}+\frac{\mathrm{i}}{2} \varepsilon_{\alpha \beta}\left(\bar{T}^{\gamma} \mathrm{D}_{\gamma}+T^{\gamma} \overline{\mathrm{D}}_{\gamma}\right) \\
& -2 \varepsilon_{\alpha \beta} \mathcal{C}^{\gamma \delta} M_{\gamma \delta}+4 \mathrm{i} \mathcal{S} M_{\alpha \beta} .
\end{aligned}
$$

Note that here the independent curvature tensor superfields $T_{\alpha}$ and $\mathcal{C}_{\alpha \beta}$ are of mass dimension $1 / 2$ and one, respectively, while both $\mathcal{S}$ and $\mathcal{R}$ are now descendants of the torsion superfields $T_{\alpha}$ and $\bar{T}_{\alpha}$.

Note that the Bianchi identities of the non-minimal algebra imply the following constraints

$$
\begin{aligned}
\overline{\mathrm{D}}_{\alpha} \mathcal{R} & =2 \mathrm{i} \bar{T}_{\alpha} \mathcal{R} \\
\mathrm{D}^{\beta} \mathcal{C}_{\alpha \beta} & =-\frac{1}{2}\left[\left(\overline{\mathrm{D}}_{\alpha}+2 \mathrm{i} \bar{T}_{\alpha}\right) \overline{\mathcal{R}}+4 \mathrm{iD} D_{\alpha} \mathcal{S}\right] .
\end{aligned}
$$

Moreover, the constraint defining a real linear superfield becomes

$$
\left(\mathrm{D}^{2}+\mathrm{i} T^{\alpha} \mathrm{D}_{\alpha}-4 \overline{\mathcal{R}}\right) G=0 .
$$

Such a constraint also holds for $\mathcal{S}$. The expression for the super-Cotton tensor expressed in terms of the non-minimal covariant derivatives $\mathrm{D}_{A}$ is

$$
\begin{aligned}
\mathcal{W}_{\alpha \beta}= & -\frac{\mathrm{i}}{4}\left[\mathrm{D}^{\gamma}, \overline{\mathrm{D}}_{\gamma}\right] \mathcal{C}_{\alpha \beta}+\frac{1}{2}\left[\mathrm{D}_{(\alpha}, \overline{\mathrm{D}}_{\beta)}\right] \mathcal{S}-\frac{1}{4} T^{\gamma} \overline{\mathrm{D}}_{(\alpha} \mathcal{C}_{\beta \gamma)}+\frac{1}{4} \bar{T}^{\gamma} \mathrm{D}_{(\alpha} \mathcal{C}_{\beta \gamma)} \\
& -\frac{1}{12} T_{(\alpha} \mathrm{D}_{\beta)} \mathcal{R}+\frac{1}{12} \bar{T}_{(\alpha} \overline{\mathrm{D}}_{\beta)} \overline{\mathcal{R}}-\frac{\mathrm{i}}{6}\left(T_{(\alpha} \overline{\mathrm{D}}_{\beta)}+\bar{T}_{(\alpha} \mathrm{D}_{\beta)}\right) \mathcal{S}+2 \mathcal{S} \mathcal{C}_{\alpha \beta},
\end{aligned}
$$

or, equivalently,

$$
\mathcal{W}_{\alpha \beta}=-\frac{1}{2}\left[\mathrm{D}_{(\alpha}, \overline{\mathrm{D}}_{\beta)}\right] \mathcal{S}+\frac{\mathrm{i}}{2} T_{(\alpha} \overline{\mathrm{D}}_{\beta)} \mathcal{S}+\frac{\mathrm{i}}{2} \bar{T}_{(\alpha} \mathrm{D}_{\beta)} \mathcal{S}-\varepsilon^{c a b}\left(\gamma_{c}\right)_{\alpha \beta} \mathrm{D}_{a} \mathcal{C}_{b}-2 \mathcal{S} \mathcal{C}_{\alpha \beta}
$$




\subsection{The superconformal setting}

In conformal superspace the conformal compensator $\Gamma$ is a primary superfield of dimension -1 and $\mathrm{U}(1)_{R}$ weight 2 ,

$$
\mathbb{D} \Gamma=-1, \quad J \Gamma=2 \Gamma, \quad K_{A} \Gamma=0,
$$

and satisfies the constraint

$$
-\frac{1}{4} \bar{\nabla}^{2} \Gamma=\lambda=\text { const. }
$$

In complete analogy to the type I and II cases, using $\Gamma$ one can introduce new covariant derivatives that take primary superfields to primary superfields. We define the new covariant derivatives $\mathscr{D}_{A}=\left(\mathscr{D}_{a}, \mathscr{D}_{\alpha}, \overline{\mathscr{D}}^{\alpha}\right)$ as follows:

$$
\begin{aligned}
& \mathscr{D}_{\alpha}:=\bar{\Gamma}^{\frac{1}{2}}\left\{\nabla_{\alpha}\right.\left.+\frac{1}{2} \nabla^{\gamma} \log (\Gamma \bar{\Gamma}) M_{\alpha \gamma}-\frac{1}{4} \nabla_{\alpha} \log \left(\frac{\Gamma}{\bar{\Gamma}}\right) J+\frac{1}{2} \nabla_{\alpha} \log (\Gamma \bar{\Gamma}) \mathbb{D}\right\} \\
& \mathscr{D}_{\alpha \beta}:=(\Gamma \bar{\Gamma})^{\frac{1}{2}}\left\{\nabla_{\alpha \beta}+\frac{\mathrm{i}}{2} \bar{\nabla}_{(\alpha} \log (\Gamma \bar{\Gamma}) \nabla_{\beta)}+\frac{\mathrm{i}}{2} \nabla_{(\alpha} \log (\Gamma \bar{\Gamma}) \bar{\nabla}_{\beta)}\right. \\
&+\frac{1}{2} \nabla_{(\alpha}{ }^{\delta} \log (\Gamma \bar{\Gamma}) M_{\beta) \delta}-\frac{\mathrm{i}}{8}\left(\nabla^{\gamma} \log (\Gamma \bar{\Gamma})\right) \bar{\nabla}_{\gamma} \log (\Gamma \bar{\Gamma}) M_{\alpha \beta} \\
&-\frac{\mathrm{i}}{8}\left[\left(\nabla_{(\alpha} \log (\Gamma \bar{\Gamma})\right) \bar{\nabla}_{\beta)} \log \left(\frac{\Gamma}{\bar{\Gamma}}\right)+\left(\bar{\nabla}_{(\alpha} \log (\Gamma \bar{\Gamma})\right) \nabla_{\beta)} \log \left(\frac{\Gamma}{\bar{\Gamma}}\right)\right] J \\
&\left.-\frac{1}{4} \nabla_{\alpha \beta} \log \left(\frac{\Gamma}{\bar{\Gamma}}\right) J+\frac{1}{2} \nabla_{\alpha \beta} \log (\Gamma \bar{\Gamma}) \mathbb{D}\right\}
\end{aligned}
$$

These covariant derivatives are such that

$$
\left[\mathbb{D}, \mathscr{D}_{A}\right]=0, \quad\left[J, \mathscr{D}_{A}\right]=0, \quad\left[K_{A}, \mathscr{D}_{B}\right\}=0,
$$

and satisfy the algebra:

$$
\begin{aligned}
\left\{\mathscr{D}_{\alpha}, \mathscr{D}_{\beta}\right\}= & -2 \mathrm{i} \mathscr{T}_{(\alpha} \mathscr{D}_{\beta)}-4 \overline{\mathscr{R}} M_{\alpha \beta}, \\
\left\{\mathscr{D}_{\alpha}, \overline{\mathscr{D}}_{\beta}\right\}= & -2 \mathrm{i} \mathscr{D}_{\alpha \beta}-\mathrm{i} \overline{\mathscr{T}}_{(\alpha} \mathscr{D}_{\beta)}+\mathrm{i} \mathscr{T}_{(\alpha} \overline{\mathscr{D}}_{\beta)}+\frac{\mathrm{i}}{2} \varepsilon_{\alpha \beta}\left(\overline{\mathscr{T}}^{\gamma} \mathscr{D}_{\gamma}+\mathscr{T}^{\gamma} \overline{\mathscr{D}}_{\gamma}\right) \\
& -2 \varepsilon_{\alpha \beta} \mathscr{C}^{\gamma \delta} M_{\gamma \delta}+4 \mathrm{i} \mathscr{S}_{\alpha \beta} .
\end{aligned}
$$

Here we have introduced the following primary dimensionless, and $\mathrm{U}(1)_{R}$ chargeless superfields

$$
\begin{aligned}
\mathscr{T}_{\alpha} & =\frac{\mathrm{i}}{4} \bar{\Gamma}^{\frac{1}{2}} \nabla_{\alpha} \log \left(\Gamma \bar{\Gamma}^{3}\right), \\
\mathscr{S} & =\frac{\mathrm{i}}{8}(\Gamma \bar{\Gamma})^{\frac{1}{2}} \nabla^{\alpha} \bar{\nabla}_{\alpha} \log (\Gamma \bar{\Gamma}), \\
\mathscr{R} & =-\frac{1}{4}\left(\Gamma^{3} \bar{\Gamma}\right)^{\frac{1}{2}} \bar{\nabla}^{2}(\Gamma \bar{\Gamma})^{-\frac{1}{2}}, \\
\mathscr{C}_{\alpha \beta} & =-\frac{1}{4}\left[\nabla_{(\alpha}, \bar{\nabla}_{\beta)}\right](\Gamma \bar{\Gamma})^{\frac{1}{2}},
\end{aligned}
$$


together with their complex conjugates. Note also that $\Gamma$ is covariantly constant with respect to the derivatives $\mathscr{D}_{A}$,

$$
\mathscr{D}_{A} \Gamma=0 .
$$

The algebra of covariant derivatives (5.21) formally coincides with that of $w=-1$ nonminimal supergravity, (5.12).

By using the compensator $\Gamma$ and the torsion superfield $\mathscr{T}_{\alpha}$, together with its descendants, we can construct general actions of the form

$$
S_{\mathrm{NM}}=\int \mathrm{d}^{3 \mid 4} z E(\Gamma \bar{\Gamma})^{-\frac{1}{2}} \mathfrak{L}(\mathscr{T}, \mathscr{D} \mathscr{T}, \cdots),
$$

where $\mathfrak{L}$ is a primary dimensionless superfield constructed out of the torsion components $\mathscr{T}$ and its covariant derivatives.

It is worth underlining the peculiarity of the presence of a primary, spinorial torsion $\mathscr{T}_{\alpha}$ in $\mathcal{N}=2$ non-minimal supergravity. Its presence is ultimately related to the fact that non-minimal supergravity has $4+4$ extra auxiliary fields compared to the minimal type I and type II cases. Consider the following independent components of the complex linear compensator

$$
\begin{aligned}
& B=\Gamma\left|, \quad \rho_{\alpha}=\nabla_{\alpha} \Gamma\right|, \quad \zeta_{\alpha}=\nabla_{\alpha} \bar{\Gamma} \mid, \\
& H=\nabla^{2} \Gamma\left|, \quad p=\bar{\nabla}^{\alpha} \nabla_{\alpha} \Gamma\right|, \quad p_{\alpha \beta}=\bar{\nabla}_{(\alpha} \nabla_{\beta)} \Gamma\left|, \quad \bar{\beta}_{\alpha}=\frac{1}{2} \nabla^{\beta} \bar{\nabla}_{\alpha} \nabla_{\beta} \Gamma\right|,
\end{aligned}
$$

together with their complex conjugates. The gauge choice

$$
\Gamma=1, \quad B_{A}=0,
$$

that fixes dilatation, $\mathrm{U}(1)_{R}$ and special conformal symmetry, at the component level corresponds to setting

$$
B=1, \quad\left(\rho_{\alpha}+\zeta_{\alpha}\right)=0, \quad b_{m}=0 .
$$

The first condition fixes the dilatation and $\mathrm{U}(1)_{R}$ symmetries. The second condition fixes $S$-supersymmetry. The last condition fixes the conformal boosts. It is clear that only half of the eight spinor components of $\zeta_{\alpha}, \bar{\zeta}_{\alpha} \rho_{\alpha}$ and $\bar{\rho}_{\alpha}$ are used to fix $S$-supersymmetry while the remainder fit in the non-minimal spinor auxiliary field

$$
T_{\alpha} \mid=\frac{1}{4}\left(\rho_{\alpha}-\zeta_{\alpha}\right)
$$

\subsection{Massive supergravity}

In this section we use the non-minimal formulation to construct a novel consistent theory of massive supergravity.

Consider the action

$$
S=\frac{1}{\tilde{\mu}} S_{\mathrm{CSG}}+S_{\text {first-order }},
$$

where $S_{\mathrm{CSG}}$ is the conformal supergravity action while $S_{\text {first-order }}$ is given by

$$
S_{\text {first-order }}=\int \mathrm{d}^{3 \mid 4} z E\left(\bar{\Phi} \Phi \mathfrak{L}(\Phi, \bar{\Phi})+\Gamma \Phi^{4}+\bar{\Gamma} \bar{\Phi}^{4}\right),
$$


and the dimensionless primary superfield $\mathfrak{L}$ is given by

$$
\mathfrak{L}:=-4-4 \mu_{2} \mathscr{R} \overline{\mathscr{R}}+4 \mu_{3} \mathscr{C}^{\alpha \beta} \mathscr{C}_{\alpha \beta}+\left(\mu_{0} \mathscr{R}^{3}+\mu_{1} \mathscr{R}^{4}+\text { c.c. }\right) \text {. }
$$

The superfields $\Gamma$ and $\bar{\Gamma}$ are the complex linear compensators defined in the previous sections and satisfy the constraint (5.18) and its conjugate, respectively. The superfields $\Phi$ and $\bar{\Phi}$ here are unconstrained complex primaries such that

$$
\mathbb{D} \Phi=\frac{1}{2} \Phi, \quad J \Phi=-\frac{1}{2} \Phi .
$$

The dimensionless primary superfields $\mathscr{R}, \overline{\mathscr{R}}$ and $\mathscr{C}_{\alpha \beta}$ are functionals of $\Phi$ and $\bar{\Phi}$ defined as (compare with (3.19))

$$
\mathscr{R}:=-\frac{1}{4 \Phi^{4}} \bar{\nabla}^{2}(\Phi \bar{\Phi}), \quad \mathscr{C}_{\alpha \beta}:=-\frac{1}{4}\left[\nabla_{(\alpha}, \bar{\nabla}_{\beta)}\right] \frac{1}{\Phi \bar{\Phi}} .
$$

An important feature of the action (5.29) is that if we vary it with respect to $\Gamma$ the Lagrange multiplier term $\Gamma \Phi^{4}$ yields the chiral constraint, $\bar{\nabla}_{\alpha} \Phi=0$. Then (5.29) with (5.31) reduces to the general type I massive action (3.52) (where for simplicity we have set $\kappa=1)$. On the other hand, we can formally integrate out the fields $\Phi$ and $\bar{\Phi}$, which upon imposing their equations of motion become functionals of $\Gamma$ and $\bar{\Gamma}$. If one then plugs the expressions for $\Phi(\Gamma, \bar{\Gamma})$ and $\bar{\Phi}(\Gamma, \bar{\Gamma})$ into eq. (5.30), the resulting model describes massive supergravity in the $\mathcal{N}=2$ non-minimal case. On-shell the resulting dynamical system is equivalent to the model described by eq. (3.52).

We have just demonstrated that, by dualizing type I models, massive supergravity can be constructed also in the non-minimal case. An open question remains whether the models obtained by dualizing the type I models are the most general massive supergravity theories one can construct in the non-minimal formulation. We leave the investigation of this question for future work.

\section{$6 \mathcal{N}=3$ supergravity with a compensating vector multiplet}

The conventional superspace formalism of [29] offers the ability to construct the most general $\mathcal{N}=3$ supergravity models. In this section we focus on $\mathcal{N}=3$ supergravity with a nowhere vanishing off-shell vector multiplet and construct various supergravity invariants up to and including curvature squared invariants. Furthermore, we will demonstrate how such invariants may be constructed within the superconformal framework. It should also be mentioned that in the context of projective superspace the $\mathcal{N}=3$ vector multiplet is often referred to as the $\mathcal{O}(2)$ multiplet. In what follows we use either name interchangeably.

\subsection{Conventional superspace}

The curved superspace $\mathcal{M}^{3 \mid 6}$ is parametrized by real bosonic $\left(x^{m}\right)$ and real fermionic $\left(\theta_{\mathrm{I}}^{\mu}\right)$ coordinates $z^{M}=\left(x^{m}, \theta_{\mathrm{I}}^{\mu}\right)$, where $m=0,1,2, \mu=1,2$, and $\mathrm{I}=1,2,3$. The superspace geometry [29] is described in terms of covariant derivatives of the form

$$
\mathcal{D}_{A}=E_{A}{ }^{M} \partial_{M}-\frac{1}{2} \Omega_{A}^{a b} M_{a b}-\frac{1}{2} \Phi_{A}{ }^{P Q} N_{P Q} .
$$


Here $E_{A}=E_{A}^{M}(z) \partial_{M}$ is the inverse supervielbein, $\Omega_{A}^{a b}$ is the Lorentz connection and $\Phi_{A} P Q$ is the $\mathrm{SO}(3)$ connection. The $\mathrm{SO}(3)$ generators $N_{I J}=-N_{J I}$ act on the spinor covariant derivatives as follows

$$
\left[N^{I J}, \mathcal{D}_{\alpha}^{K}\right]=2 \delta^{K[I} \mathcal{D}_{\alpha}^{J]}
$$

The supergravity spinor covariant derivatives obey the following anti-commutation relations:

$$
\begin{aligned}
\left\{\mathcal{D}_{\alpha}^{I}, \mathcal{D}_{\beta}^{J}\right\}= & 2 \mathrm{i} \delta^{I J}\left(\gamma^{c}\right)_{\alpha \beta} \mathcal{D}_{c}-2 \mathrm{i} \varepsilon_{\alpha \beta} \mathcal{C}^{\gamma \delta I J} M_{\gamma \delta}-4 \mathrm{i}\left(\mathcal{S}^{I J}+\delta^{I J} \mathcal{S}\right) M_{\alpha \beta} \\
+ & {\left[-4 \mathrm{i} \varepsilon_{\alpha \beta} \mathcal{S}^{K[I} \delta^{J] L}-4 \mathrm{i} \varepsilon_{\alpha \beta} \delta^{K[I} \delta^{J] L} \mathcal{S}+\mathrm{i} \mathcal{C}_{\alpha \beta}{ }^{K L} \delta^{I J}\right.} \\
& \left.-4 \mathrm{i} \mathcal{C}_{\alpha \beta}{ }^{K(I} \delta^{J) L}\right] N_{K L},
\end{aligned}
$$

where $\mathcal{S}^{I J}$ and $\mathcal{C}_{\alpha \beta}{ }^{I J}$ satisfy the symmetry properties

$$
\mathcal{S}^{I J}=\mathcal{S}^{(I J)}, \quad \mathcal{S}^{I}{ }_{I}=0, \quad \mathcal{C}_{\alpha \beta}{ }^{I J}=\mathcal{C}_{\alpha \beta}{ }^{[I J]}=\mathcal{C}_{(\alpha \beta)}{ }^{I J}
$$

as well as the Bianchi identities

$$
\begin{aligned}
\mathcal{D}_{\alpha}^{I} C_{\beta \gamma}{ }^{J K}= & \frac{2}{3} \varepsilon_{\alpha(\beta}\left(\mathcal{C}_{\gamma)}{ }^{I J K}+3 \mathcal{T}_{\gamma)}{ }^{J K I}+4\left(\mathcal{D}_{\gamma)}^{[J} \mathcal{S}\right) \delta^{K] I}-\frac{1}{3} \mathcal{S}_{\gamma)}{ }^{[J} \delta^{K] I}\right) \\
& +\mathcal{C}_{\alpha \beta \gamma}{ }^{I J K}-2 \mathcal{C}_{\alpha \beta \gamma}{ }^{[J} \delta^{K] I} \\
\mathcal{D}_{\alpha}^{I} \mathcal{S}^{J K}= & 2 \mathcal{T}_{\alpha}{ }^{I(J K)}+\mathcal{S}_{\alpha}{ }^{(J} \delta^{K) I}-\frac{1}{3} \mathcal{S}_{\alpha}{ }^{I} \delta^{J K}
\end{aligned}
$$

The symmetry properties of $\mathcal{C}_{\alpha}{ }^{I J K}, \mathcal{C}_{\alpha \beta \gamma}{ }^{I J K}$ and $\mathcal{C}_{\alpha \beta \gamma}{ }^{I}$ are

$$
\mathcal{C}_{\alpha}{ }^{I J K}=\mathcal{C}_{\alpha}{ }^{[I J K]}, \quad \mathcal{C}_{\alpha \beta \gamma}{ }^{I J K}=\mathcal{C}_{\alpha \beta \gamma}{ }^{[I J K]}=\mathcal{C}_{(\alpha \beta \gamma)}{ }^{I J K}, \quad \mathcal{C}_{\alpha \beta \gamma}{ }^{I}=\mathcal{C}_{(\alpha \beta \gamma)}{ }^{I},
$$

while the superfield $\mathcal{T}_{\alpha}^{I J K}$ is such that

$$
\mathcal{T}_{\alpha}^{I J K}=\mathcal{T}_{\alpha}^{[I J] K}, \quad \delta_{J K} \mathcal{T}_{\alpha}{ }^{I J K}=\mathcal{T}_{\alpha}^{[I J K]}=0 .
$$

The remaining covariant derivative commutation relations follow from the spinor covariant derivative anti-commutator, see [29] for more details.

The superspace geometry describes conformal supergravity because it admits superWeyl transformations of the form [69]

$$
\begin{aligned}
& \mathcal{D}_{\alpha}^{\prime I}= \mathrm{e}^{\frac{1}{2} \sigma}\left(\mathcal{D}_{\alpha}^{I}+\mathcal{D}^{\beta I} \sigma M_{\alpha \beta}+\mathcal{D}_{\alpha J} \sigma N^{I J}\right) \\
& \mathcal{D}_{a}^{\prime}=\mathrm{e}^{\sigma}\left(\mathcal{D}_{a}+\frac{\mathrm{i}}{2}\left(\gamma_{a}\right)^{\alpha \beta}\left(\mathcal{D}_{\alpha}^{K} \sigma\right) \mathcal{D}_{\beta K}+\varepsilon_{a b c}\left(\mathcal{D}^{b} \sigma\right) M^{c}+\frac{\mathrm{i}}{4}\left(\mathcal{D}_{K}^{\rho} \sigma\right) \mathcal{D}_{\rho}^{K} \sigma M_{a}\right. \\
&\left.\quad+\frac{\mathrm{i}}{48}\left(\gamma_{a}\right)^{\alpha \beta} \mathrm{e}^{-3 \sigma}\left[\mathcal{D}_{\alpha}^{K}, \mathcal{D}_{\beta}^{L}\right] \mathrm{e}^{3 \sigma}\right) .
\end{aligned}
$$


The corresponding transformations of the torsion superfields are

$$
\begin{aligned}
\mathcal{S}^{\prime} & =-\frac{\mathrm{i}}{6} \mathrm{e}^{\frac{1}{2} \sigma}\left(\mathcal{D}_{K}^{\gamma} \mathcal{D}_{\gamma}^{K}+6 \mathrm{i} \mathcal{S}\right) \mathrm{e}^{\frac{1}{2} \sigma}, \\
\mathcal{S}^{\prime I J} & =\frac{\mathrm{i}}{4} \mathrm{e}^{2 \sigma}\left(\mathcal{D}^{\gamma I} \mathcal{D}_{\gamma}^{J}-\frac{1}{3} \delta^{I J} \mathcal{D}_{K}^{\gamma} \mathcal{D}_{\gamma}^{K}-4 \mathrm{i} \mathcal{S}^{I J}\right) \mathrm{e}^{-\sigma}, \\
\mathcal{C}_{\alpha \beta}^{\prime}{ }^{I J} & =\frac{\mathrm{i}}{2}\left(\mathcal{D}_{(\alpha}^{[I} \mathcal{D}_{\beta)}^{J]}-2 \mathrm{i} \mathcal{C}_{\alpha \beta}{ }^{I J}\right) \mathrm{e}^{\sigma} .
\end{aligned}
$$

All supergravity-matter invariants can be made super-Weyl invariant with the use of the vector multiplet compensator field strength $G^{I}$, which satisfies the Bianchi identity

$$
\mathcal{D}_{\alpha}^{(I} G^{J)}=\frac{1}{3} \delta^{I J} \mathcal{D}_{\alpha}^{K} G_{K} .
$$

One only needs to make use of its magnitude $G$,

$$
G^{2}:=G^{I} G_{I},
$$

which transforms homogeneously under super-Weyl transformations,

$$
G^{\prime}=\mathrm{e}^{\sigma} G .
$$

It should be mentioned that the abelian vector multiplet is also known as the $\mathcal{O}(2)$ multiplet. The reason for this is made evident by making use of the isomorphism $\mathrm{SO}(3) \cong$ $\mathrm{SU}(2) / \mathbb{Z}_{2}$ and replacing any $\mathrm{SO}(3)$ vector index by a symmetric pair of $\mathrm{SU}(2)$ spinor indices. For the isovector $G^{I}$, one can instead work with the symmetric spinor $G^{i j}$ defined by

$$
G^{I}:=\left(\Sigma^{I}\right)_{i j} G^{i j}, \quad G_{i j}=\left(\Sigma_{I}\right)_{i j} G^{I},
$$

where the sigma-matrices are given by

$$
\left(\Sigma_{I}\right)_{i j}:=\left(\mathbb{1}, \mathrm{i} \sigma_{1}, \mathrm{i} \sigma_{3}\right)=\left(\Sigma_{I}\right)_{j i}
$$

The $\mathcal{O}(2)$ multiplet can be shown to satisfy the analyticity constraint

$$
\mathcal{D}_{\alpha}^{(i j} G^{k l)}=0,
$$

where $\mathcal{D}_{\alpha}^{i j}:=\left(\Sigma_{I}\right)^{i j} \mathcal{D}_{\alpha}^{I}$. The isospinor representation of isotensors is used in the projective superspace formulation [29].

General off-shell matter couplings in $\mathcal{N}=3$ supergravity were constructed in [29] by using projective superspace. Given a supergravity-matter system, its dynamics can be described by a Lagrangian $\mathcal{L}^{(2)}(v)$ which is a real weight-two covariant projective supermultiplet, ${ }^{18}$ with $v^{i}$ the homogeneous coordinates for $\mathbb{C} P^{1}$. We refer the reader to [29] for the definition and notations of $\mathcal{N}=3$ covariant projective superspace and details about the supersymmetric action principle in that context. See also [19] for the generalization to the $\mathcal{N}=3$ conformal superspace of [30].

It was shown in [19] that in the presence of an Abelian vector multiplet with nowhere vanishing gauge invariant field strength $G^{i j}, G:=\sqrt{G^{i j} G_{i j}} \neq 0$, the action functional can be rewritten as a $B F$ term for a composite real $\mathcal{O}(2)$ multiplet $G^{i j}$. In this sense the $B F$ action may be used as a universal action principle for $\mathcal{N}=3$. We will discuss the $B F$ action in more detail below in the superconformal context.

\footnotetext{
${ }^{18}$ In what follows, we suppress explicit $z$-dependence of $\mathcal{N}=3$ superfields.
} 


\subsection{The superconformal setting}

Since our actions can be made to be superconformally invariant it is natural to work with a manifestly superconformal framework. In this subsection we introduce such a framework and provide the ingredients for the construction of supergravity invariants.

\subsubsection{The abelian vector multiplet}

Here we make use of $\mathcal{N}=3$ conformal superspace described in appendix A. The abelian vector multiplet superfield strength $G^{I}$ is a dimension 1 primary,

$$
\mathbb{D} G^{I}=G^{I}, \quad K_{A} G^{I}=0,
$$

and satisfies the Bianchi identity

$$
\nabla_{\alpha}^{(I} G^{J)}=\frac{1}{3} \delta^{I J} \nabla_{\alpha}^{K} G_{K}
$$

The superfield $G^{I}$ naturally appears in the components of the gauge-invariant field strength $F=\mathrm{d} V$,

$$
F=\frac{1}{2} E_{J}^{\beta} \wedge E_{I}^{\alpha} F_{\alpha \beta}^{I J}+E_{J}^{\beta} \wedge E^{a} F_{a \beta}^{J}+\frac{1}{2} E^{b} \wedge E^{a} F_{a b},
$$

where

$$
\begin{aligned}
F_{\alpha \beta}^{I J} & =-2 \mathrm{i} \varepsilon_{\alpha \beta} \varepsilon^{I J K} G_{K}, \\
F_{a \alpha}^{I} & =\frac{1}{2}\left(\gamma_{a}\right)_{\alpha}{ }^{\beta} \varepsilon^{I J K} \nabla_{\beta J} G_{K}, \\
F_{a b} & =-\frac{\mathrm{i}}{24} \varepsilon_{a b c}\left(\gamma^{c}\right)^{\alpha \beta} \varepsilon_{I J K}\left[\nabla_{\alpha}^{I}, \nabla_{\beta}^{J}\right] G^{K} .
\end{aligned}
$$

Using $G=\sqrt{G^{I} G_{I}}$ one can construct dimensionless covariant derivatives that take primary superfields to primary superfields. The covariant derivatives are

$$
\begin{aligned}
& \mathscr{D}_{\alpha}^{I}=G^{-\frac{1}{2}}\left(\nabla_{\alpha}^{I}-\left(\nabla^{\beta I} \ln G\right) M_{\alpha \beta}-\left(\nabla_{\alpha J} \ln G\right) N^{I J}-\left(\nabla_{\alpha}^{I} \ln G\right) \mathbb{D}\right), \\
& \mathscr{D}_{a}=\frac{\mathrm{i}}{12}\left(\gamma_{a}\right)^{\alpha \beta}\left\{\mathscr{D}_{\alpha}^{K}, \mathscr{D}_{\beta K}\right\}+2 \mathscr{S} M_{a}+\frac{1}{6} \mathscr{C}_{a}{ }^{K L} N_{K L},
\end{aligned}
$$

where we have introduced the dimensionless primary superfields

$$
\begin{aligned}
\mathscr{C}_{a}{ }^{I J} & :=-\frac{\mathrm{i}}{4}\left(\gamma_{a}\right)^{\alpha \beta} \nabla_{\alpha}^{[I} \nabla_{\beta}^{J]} \frac{1}{G} \equiv \varepsilon^{I J K} \mathscr{C}_{a K}, \\
\mathscr{S} & :=-\frac{\mathrm{i}}{6 G^{\frac{1}{2}}} \nabla_{K}^{\gamma} \nabla_{\gamma}^{K} G^{-\frac{1}{2}} .
\end{aligned}
$$

It can be checked that the covariant derivatives annihilate $G, \mathscr{D}_{A} G=0$. On primary superfields one can verify that the covariant derivatives (6.20) satisfy the algebra

$$
\begin{aligned}
\left\{\mathscr{D}_{\alpha}^{I}, \mathscr{D}_{\beta}^{J}\right\}= & 2 \mathrm{i} \delta^{I J}\left(\gamma^{c}\right)_{\alpha \beta} \mathscr{D}_{c}-2 \mathrm{i} \varepsilon_{\alpha \beta} \mathscr{C}^{\gamma \delta I J} M_{\gamma \delta}-4 \mathrm{i}\left(\mathscr{S}^{I J}+\delta^{I J} \mathscr{S}\right) M_{\alpha \beta} \\
+ & \left(-4 \mathrm{i} \varepsilon_{\alpha \beta} \mathscr{S}^{K[I} \delta^{J] L}-4 \mathrm{i} \varepsilon_{\alpha \beta} \delta^{K[I} \delta^{J] L} \mathscr{S}+\mathrm{i} \mathscr{C}_{\alpha \beta}{ }^{K L} \delta^{I J}\right. \\
& \left.-4 \mathrm{i} \mathscr{C}_{\alpha \beta}{ }^{K(I} \delta^{J) L}\right) N_{K L},
\end{aligned}
$$


where we have introduced

$$
\mathscr{S}^{I J}:=\frac{\mathrm{i}}{4 G^{2}}\left(\nabla^{\gamma(I} \nabla_{\gamma}^{J)}-\frac{1}{3} \delta^{I J} \nabla_{K}^{\gamma} \nabla_{\gamma}^{K}\right) G .
$$

The algebra of covariant derivative of $\mathscr{D}_{A}$ can be seen to formally agree with the one of $\mathrm{SO}(3)$ superspace. It is worth mentioning that in the gauge $B_{A}=0$ the superfields $\mathscr{S}^{I J}$, $\mathscr{S}$ and $\mathscr{C}_{\alpha \beta}{ }^{I J}$ become

$$
\begin{aligned}
\mathscr{C}_{\alpha \beta}{ }^{I J} & =\frac{\mathrm{i}}{2}\left(\mathcal{D}_{(\alpha}^{[I} \mathcal{D}_{\beta)}^{J]}-2 \mathrm{i} \mathcal{C}_{\alpha \beta}{ }^{I J}\right) \frac{1}{G}, \\
\mathscr{S} & =-\frac{\mathrm{i}}{6 G^{\frac{1}{2}}}\left(\mathcal{D}_{K}^{\gamma} \mathcal{D}_{\gamma}^{K}+6 \mathrm{i} \mathcal{S}\right) \frac{1}{G^{\frac{1}{2}}}, \\
\mathscr{S}^{I J} & =\frac{\mathrm{i}}{4 G^{2}}\left(\mathcal{D}^{\gamma I} \mathcal{D}_{\gamma}^{J}-\frac{1}{3} \delta^{I J} \mathcal{D}_{K}^{\gamma} \mathcal{D}_{\gamma}^{K}-4 \mathrm{i} \mathcal{S}^{I J}\right) G .
\end{aligned}
$$

Similar to the previous cases the superfields $\mathscr{S}^{I J}, \mathscr{S}$ and $\mathscr{C}_{\alpha \beta}{ }^{I J}$ can be seen to degauge to $\mathcal{S}^{I J}, \mathcal{S}$ and $C_{\alpha \beta}{ }^{I J}$ upon imposing the gauge condition $G=1$.

Instead of working with $G^{I}$, one can also equivalently make use of the prescription (6.13) and introduce $G^{(2)}$ defined such that

$$
\nabla_{\alpha}^{(2)} G^{(2)}=0 \quad \Longleftrightarrow \quad \nabla_{\alpha}^{(i j} G^{k l)}=0, \quad G^{(2)}(v):=G_{i j} v^{i} v^{j}, \quad \nabla_{\alpha}^{(2)}:=v_{i} v_{j} \nabla_{\alpha}^{i j},
$$

where $v^{i}$ are homogeneous coordinates for $\mathbb{C} P^{1} \cdot{ }^{19}$ In this form the $\mathcal{O}(2)$ multiplet can be given the prepotential realisation [29]

$$
G^{(2)}(v):=G_{i j} v^{i} v^{j}=\Delta^{(4)} \oint_{\hat{\gamma}} \frac{(\hat{v}, \mathrm{~d} \hat{v})}{2 \pi(v, \hat{v})^{2}} \mathcal{V}(\hat{v})
$$

where $\mathcal{V}(v)$ is the tropical prepotential for the vector multiplet, $\nabla_{\alpha}^{(2)} \mathcal{V}=0$, and we have introduced the analytic projection operator [29]

$$
\Delta^{(4)}:=\frac{\mathrm{i}}{4} \nabla^{\alpha(2)} \nabla_{\alpha}^{(2)}
$$

Here the prepotential $\mathcal{V}$ possesses the gauge transformations

$$
\delta \mathcal{V}=\lambda+\breve{\lambda}, \quad \nabla_{\alpha}^{(2)} \lambda=0,
$$

where the gauge parameter $\lambda$ is an arbitrary weight- 0 arctic multiplet and $\breve{\lambda}$ is its smileconjugate, see [29] for more details.

A noteworthy and useful composite $\mathcal{O}(2)$ multiplet $\mathbb{G}^{(2)}$ may be constructed using the prepotential realisation (6.26) with the prepotential [29]

$$
\mathcal{V}=\ln \left(\frac{G^{(2)}}{i \Upsilon^{(1)} \breve{\Upsilon}^{(1)}}\right)
$$

\footnotetext{
${ }^{19}$ Refer to $[19,29]$ for details on the formalism of projective superspace.
} 
where $\Upsilon^{(1)}$ is a weight- 1 arctic multiplet and $\breve{\Upsilon}^{(1)}$ its smile conjugate [29]. The composite $\mathbb{G}^{I}$, which was used in the description of $(2,1)$ AdS supergravity [19], can be expressed in terms of the composites $\mathscr{S}^{I J}$ and $\mathscr{S}$ as follows:

$$
\begin{aligned}
\mathbb{G}^{I} & =4\left(\mathscr{S}^{I J}+\delta^{I J} \mathscr{S}\right) G_{J}, \\
& =\frac{\mathrm{i}}{G^{2}} G_{J}\left(\nabla^{\gamma(I} \nabla_{\gamma}^{J)}-\frac{1}{3} \delta^{I J} \nabla_{K}^{\gamma} \nabla_{\gamma}^{K}\right) G-\frac{2 \mathrm{i}}{3 G^{\frac{1}{2}}} G^{I} \nabla_{K}^{\gamma} \nabla_{\gamma}^{K} G^{-\frac{1}{2}}
\end{aligned}
$$

It will be a useful ingredient in the construction of supergravity invariants.

\subsubsection{The $B F$ action}

General off-shell matter couplings in $\mathcal{N}=3$ supergravity were constructed in [29] and it was shown in [19] that the general action functional there can be rewritten as a $B F$ term

$$
\begin{aligned}
S_{\mathrm{LM}} & =\frac{1}{2 \pi \mathrm{i}} \oint_{\gamma}(v, \mathrm{~d} v) \int \mathrm{d}^{3 \mid 6} z E C^{(-4)} \mathcal{L}^{(2)} \\
& =\frac{1}{2 \pi \mathrm{i}} \oint_{\gamma}(v, \mathrm{~d} v) \int \mathrm{d}^{3 \mid 6} z E C^{(-4)} \mathcal{V} G^{(2)}, \quad \mathrm{d}^{3 \mid 6} z=\mathrm{d}^{3} x \mathrm{~d}^{6} \theta,
\end{aligned}
$$

where $\mathcal{V}(v)$ is the tropical prepotential for the $\mathcal{O}(2)$ multiplet $G^{(2)}$, while $\boldsymbol{G}^{(2)}$ is some composite $\mathcal{O}(2)$ multiplet. Here the model-independent isotwistor superfield $C^{(-4)}(v)$ of weight -4 is required to be conformally primary and of dimension -1 . The action (6.31) is invariant under the gauge transformations (6.28). The action (6.31) is also called the $\mathcal{N}=3$ linear multiplet action. The component form of the action (6.31) is [19]

$$
\begin{aligned}
S_{\mathrm{LM}}= & \frac{1}{2} \int \mathrm{d}^{3} x e\left(\varepsilon^{a b c} v_{a} \boldsymbol{f}_{b c}-2 \mathrm{i} \chi^{\gamma} \boldsymbol{\chi}_{\gamma}-\frac{\mathrm{i}}{4} \lambda^{\gamma I J} \boldsymbol{\lambda}_{\gamma I J}+g^{I} \boldsymbol{h}_{I}+\boldsymbol{g}^{I} h_{I}\right. \\
& -\frac{1}{2}\left(\gamma^{a}\right)_{\gamma \delta} \psi_{a_{I}^{\gamma}}^{\gamma}\left(\lambda^{\delta I J} \boldsymbol{g}_{J}+\boldsymbol{\lambda}^{\delta I J} g_{J}+\mathrm{i} \chi^{\delta} \boldsymbol{g}^{I}+\mathrm{i} \chi^{\delta} g^{I}\right) \\
& \left.+\frac{\mathrm{i}}{2} \varepsilon^{a b c}\left(\gamma_{a}\right)_{\gamma \delta} \psi_{b}^{\gamma} \psi_{c L}^{\delta}\left(\delta^{K L} g^{P} \boldsymbol{g}_{P}-2 g^{K} \boldsymbol{g}^{L}\right)\right),
\end{aligned}
$$

where the component fields are defined as follows:

$$
\begin{aligned}
g_{I} & =G_{I}\left|, \quad \lambda_{\alpha}^{I J}=2 \nabla_{\alpha}^{[I} G^{J]}\right|, \quad \chi_{\alpha}=\frac{\mathrm{i}}{3} \nabla_{\alpha}^{I} G_{I}\left|, \quad h_{I}=-\mathrm{i} \nabla^{\gamma J} \nabla_{\gamma J} G_{I}\right| \\
v_{a} & =e_{a}{ }^{m} V_{m}\left|=V_{a}\right|+\frac{1}{2} \psi_{a I}^{\alpha} V_{\alpha}^{I} \mid \\
f_{a b} & =F_{a b} \mid-\frac{1}{2}\left(\psi_{[a}^{K} \gamma_{b]} \lambda_{K}\right)+\frac{\mathrm{i}}{2} \psi_{a}^{\gamma K} \psi_{b \gamma}^{L} g_{K L} \\
& =-\frac{\mathrm{i}}{12} \varepsilon_{a b c}\left(\gamma^{c}\right)^{\alpha \beta} \varepsilon^{I J K} \nabla_{\alpha I} \nabla_{\beta J} G_{K} \mid-\frac{1}{4} \varepsilon^{I J K}\left(\psi_{[a I} \gamma_{b]} \lambda_{J K}\right)+\frac{\mathrm{i}}{2} \varepsilon^{I J K} \psi_{a I}^{\gamma} \psi_{b \gamma J} g_{K}
\end{aligned}
$$

The same definitions hold for the component fields of $\boldsymbol{G}$.

The component fields of the Weyl multiplet were defined in [30]. The vielbein $e_{m}{ }^{a}$, the gravitino $\psi_{m_{I}}^{\alpha}$, the $\mathrm{SO}(3)$ gauge field $V_{m}{ }^{I J}$ and the dilatation gauge field $b_{m}$, are defined as the lowest components of their corresponding superforms,

$$
e_{m}^{a}:=E_{m}{ }^{a}\left|, \quad \psi_{m_{I}}^{\alpha}:=2 E_{m_{I}}^{\alpha}\right|, \quad V_{m}^{I J}:=\Phi_{m}^{I J}\left|, \quad b_{m}:=B_{m}\right| .
$$


There also exists an additional component field $w_{\alpha}$ which is defined as the component projection of the super-Cotton tensor $W_{\alpha}, w_{\alpha}=W_{\alpha} \mid$. At the component level, we will be mostly interested in the bosonic sector of locally supersymmetric invariants and therefore will not be concerned with fermionic fields.

To fix the dilatations, $S$-supersymmetry, special conformal symmetry and $\mathrm{SO}(3)$ symmetry in our invariants one can make use of the gauge conditions

$$
G^{I}=(0,0, G), \quad G=1, \quad B_{A}=0
$$

which leads to the following conditions at the component level:

$$
g^{I}=(0,0, g), \quad g:=\sqrt{g^{I} g_{I}}=1, \quad \chi_{\alpha}=\lambda_{\alpha}{ }^{I J}=0, \quad b_{m}:=B_{m} \mid=0 .
$$

The first breaks the $\mathrm{SO}(3)$ symmetry, the second breaks dilatations, the third fixes $S$ supersymmetry transformations and the last fixes the special conformal transformations. Note that the gauge conditions (6.36) also imply that

$$
h^{I} \equiv(0,0, h) .
$$

In the gauge (6.36) one can verify the following useful results:

$$
\begin{aligned}
\square g^{I} & =\frac{1}{4} R g^{I}+\text { fermion terms }, \\
\square^{2} g^{I} & =R^{a b} R_{a b} g^{I}-\frac{1}{8} R^{2} g^{I}+\frac{1}{4}\left(D^{a} D_{a} R\right) g^{I}+\text { fermion terms },
\end{aligned}
$$

where we have defined

$$
\begin{aligned}
\square g^{I} & :=\nabla^{a} \nabla_{a} G^{I} \mid \\
\square^{2} g^{I} & :=\nabla^{a} \nabla_{a} \nabla^{b} \nabla_{b} G^{I} \mid
\end{aligned}
$$

and

$$
D_{a}=e_{a}{ }^{m}\left(\partial_{m}-\frac{1}{2} \omega_{m}{ }^{b c} M_{b c}-V_{m}{ }^{K L} N_{K L}-b_{m} \mathbb{D}\right) .
$$

The above results will be useful in identifying the curvature terms in the invariants at the component level.

\subsection{Supergravity invariants}

In this subsection we construct various supergravity invariants in superspace using the composites $\mathscr{S}^{I J}$ and $\mathscr{S}$.

\subsubsection{The supergravity action}

The $\mathcal{N}=(2,1)$ supergravity action with a cosmological term is described by the Lagrangian [29]

$$
\mathcal{L}^{(2)}=\frac{1}{\kappa} \mathcal{L}_{\mathrm{SG}}^{(2)}+\lambda \mathcal{L}_{\mathrm{cos}}^{(2)},
$$


where

$$
\begin{aligned}
\mathcal{L}_{\mathrm{SG}}^{(2)} & =G^{(2)} \ln \left(\frac{G^{(2)}}{\mathrm{i} \Upsilon^{(1)} \breve{\Upsilon}^{(1)}}\right), \\
\mathcal{L}_{\mathrm{cos}}^{(2)} & =\mathcal{V} G^{(2)} .
\end{aligned}
$$

The component action corresponding to $\mathcal{L}_{\mathrm{SG}}^{(2)}$ can be constructed by letting $G^{I} \rightarrow \mathbb{G}^{I}$ in the component $B F$ action (6.32). After doing so, one can verify that the component field $\boldsymbol{h}^{I}$ contains a term proportional to $\frac{1}{g} \square g^{I}$. Imposing the gauge condition (6.36) and using the results (6.38) recovers the Einstein-Hilbert term in the action. The cosmological term coming from $\mathcal{L}_{\text {cos }}^{(2)}$ corresponds to a U(1) Chern-Simons term. The equations of motion for the supergravity model was given in [19].

\subsubsection{The $R h^{n}$ invariants}

An invariant can be constructed using

$$
\mathcal{L}_{R h^{n}}^{(2)}=\left(\frac{\mathbb{G}^{(2)}}{G^{(2)}}\right)^{n} G^{(2)}
$$

Using integration by parts one can show that the corresponding action can be equivalently constructed from

$$
\mathcal{L}^{(2)}=\mathcal{V} \mathbb{G}_{n}^{(2)},
$$

where we have defined [19]

$$
\mathbb{G}_{n}^{(2)}:=\Delta^{(4)} \oint_{\hat{\gamma}} \frac{(\hat{v}, \mathrm{~d} \hat{v})}{2 \pi(v, \hat{v})^{2}}\left(\frac{\mathbb{G}^{(\hat{2})}}{G^{(\hat{2})}}\right)^{n}
$$

The component action corresponding to $\mathcal{L}_{R h^{n}}^{(2)}$ may be obtained by letting $\boldsymbol{G}^{I} \rightarrow \mathbb{G}^{I}$ in the component $B F$ action (6.32). For $n=1$ the action can be seen to vanish since upon integrating by parts it coincides with

$$
\mathcal{L}^{(2)}=\mathbb{G}^{(2)}
$$

which may be thought of as a $B F$ action with one of the vector multiplets set to zero. For $n \geq 2$ it can be shown to contain a term proportional to $R h^{n}$ upon imposing the gauge conditions (6.36).

For the $n=1$ case one can use the following independent invariant

$$
\mathcal{L}_{R h}^{(2)}=\mathbb{G}^{(2)} \ln \left(\frac{G^{(2)}}{\mathrm{i} \Upsilon^{(1)} \breve{\Upsilon}^{(1)}}\right)
$$

The component action can be analysed by putting $G^{I} \rightarrow \mathbb{G}^{I}$ and $G^{I} \rightarrow \mathbb{G}^{I}$ into the $B F$ action. A term of the form

$$
\frac{1}{g^{2}} h_{I} \square g^{I}
$$


will arise in the component action in the contribution $g^{I} \boldsymbol{h}_{I}+\boldsymbol{g}^{I} h_{I}$. Upon gauge fixing it gives rise to a $R h$ term. In analogy with the $\mathcal{N}=2$ case with a vector multiplet we call the invariant a $R h$ invariant.

It is also worth mentioning that upon integrating by parts one can show that the supergravity invariant corresponding to $\mathcal{L}_{R h}^{(2)}$ can be described by the Lagrangian

$$
\mathcal{L}^{(2)}=V \mathbb{G}_{0}^{(2)},
$$

where

$$
\mathbb{G}_{0}^{(2)}=\Delta^{(4)} \oint_{\hat{\gamma}} \frac{(\hat{v}, \mathrm{~d} \hat{v})}{2 \pi(v, \hat{v})^{2}} \frac{\mathbb{G}^{(\hat{2})}}{G^{(\hat{2})}} \ln \left(\frac{G^{(\hat{2})}}{\mathrm{i} \Upsilon^{(\hat{1})} \breve{\Upsilon}^{(\hat{1})}}\right)
$$

\subsubsection{Ricci squared invariants}

In the previous subsections it was straightforward to write down the superspace Lagrangian $\mathcal{L}^{(2)}$ for various invariants. To construct a Ricci squared invariant we will instead adopt a different approach and first construct an invariant in full superspace. To construct locally supersymmetric invariants one can use the conventional $\mathcal{N}=3$ locally supersymmetric action

$$
S=\mathrm{i} \int \mathrm{d}^{3 \mid 6} z E \mathcal{L}
$$

where the Lagrangian $\mathcal{L}$ is a dimensionless primary scalar superfield

$$
\mathbb{D} \mathcal{L}=0, \quad K_{A} \mathcal{L}=0
$$

It was shown in [19] that the above action can be recast in the form (6.31) with

$$
\mathcal{L}^{(2)}=2 \Delta^{(4)} \frac{G \mathcal{L}}{G^{(2)}}
$$

and

$$
\boldsymbol{G}^{(2)}=\Delta^{(4)} \oint_{\hat{\gamma}} \frac{(\hat{v}, \mathrm{~d} \hat{v})}{2 \pi(v, \hat{v})^{2}} \frac{\mathcal{L}^{(2)}(\hat{v})}{G^{(2)}(\hat{v})}
$$

One can consider the general actions of the form

$$
S=\mathrm{i} \int \mathrm{d}^{3 \mid 6} z E \mathfrak{L}\left(\mathscr{T}, \mathscr{D} \mathscr{T}, \mathscr{D}^{2} \mathscr{T}, \cdots\right),
$$

where $\mathfrak{L}$ is a dimensionless superfield constructed out of the torsion components $\mathscr{T}$ of the covariant derivatives $\mathscr{D}_{A}$. One can in principle construct general higher derivatives couplings beyond those of the previous subsections using the action (6.55). As in the previous sections we will focus our attention on actions containing at most curvature squared terms and thus search for an invariant containing a Ricci squared term.

To construct a Ricci squared term we will consider the invariant

$$
S=\mathrm{i} \int \mathrm{d}^{3 \mid 6} z E \mathcal{L}_{\zeta, \rho},
$$


with

$$
\mathcal{L}_{\zeta, \rho}=\frac{\zeta}{G^{2}} \mathscr{S}^{I J} G_{I} G_{J}+\rho \mathscr{S}
$$

and $\zeta$ and $\rho$ are arbitrary constants. Using eq. (6.53) and (6.54) we can rewrite the full superspace action in terms of a $B F$ action with the composite $\mathcal{O}(2)$ multiplet

$$
\boldsymbol{G}^{(2)}(v)=2 \Delta^{(4)} \oint_{\hat{\gamma}} \frac{(\hat{v}, \mathrm{~d} \hat{v})}{2 \pi(v, \hat{v})^{2}} \frac{1}{\left(G^{(2)}(\hat{v})\right)^{2}} \Delta^{(\hat{4})}(\hat{v})\left(G \mathcal{L}_{\zeta, \rho}\right)
$$

It can be shown that the above composite for $\zeta=0$ or $\rho=0$ leads to a Ricci curvature squared term in the action since the action will contain a term proportional to $\frac{1}{g^{3}} g_{I} \square^{2} g^{I}$. Furthermore, one can show that the Ricci curvature squared contributions cancel for $\rho=4 \zeta$. However, the corresponding invariant does not coincide with a linear combination of the previous invariants. Thus we expect that by using the action corresponding to $\mathcal{L}_{1,4}$ one can construct an invariant containing a scalar curvature squared contribution independent of the $R h^{2}$ invariant already constructed.

It should be mentioned that since $\mathcal{L}$ is dimensionless we can let $\mathcal{L}=1$ without breaking the dilatation symmetry. Upon doing so it is not immediately obvious whether the invariant (6.51) gives another non-vanishing invariant, which is a full superspace volume. However, it is not difficult to verify that it does indeed vanish. To see this we construct the action using the superspace Lagrangian

$$
\mathcal{L}^{(2)}=2 \Delta^{(4)} \frac{G}{G^{(2)}} .
$$

One can check that it is proportional to the composite $\mathbb{G}^{(2)}$,

$$
\mathcal{L}^{(2)}=2 \mathbb{G}^{(2)} .
$$

From which it is clear that the full superspace volume must vanish,

$$
\int \mathrm{d}^{3 \mid 6} z E=0 .
$$

It would be interesting to re-derive our result (6.61) using the normal coordinates techniques of [70]. Eq. (6.61) is actually quite remarkable. The point is that there exist only three conformal supergravity theories for which one can define a full superspace volume (without use of any compensator), specifically: $2 \mathrm{D} \mathcal{N}=(2,2), 3 \mathrm{D} \mathcal{N}=3$ and $4 \mathrm{D} \mathcal{N}=2$. It is only in these cases that the superspace measure is dimensionless, and therefore invariant under the super-Weyl transformations. In the $2 \mathrm{D} \mathcal{N}=(2,2)$ case, the corresponding full superspace volume is vanishing, as follows from eq. (4.1) in [71]. In the $4 \mathrm{D} \mathcal{N}=2$ case, the full superspace volume also vanishes, as follows from eqs. (3.22) and (3.23) in [70]. Eq. (6.61) tells us that this property holds in the remaining case, and is therefore generic. It should be remarked that the $3 \mathrm{D} \mathcal{N}=2$ property

$$
S=\int \mathrm{d}^{3 \mid 4} z E G=0
$$

can also be interpreted as the vanishing superspace volume in type II supergravity provided one makes use of the covariant derivatives (4.11), which is analogous to the new minimal formulation for $4 \mathrm{D} \mathcal{N}=1$ supergravity [72]. However, the latter result holds in the presence of a conformal compensator and thus corresponds to Poincaré supergravity. 


\section{Concluding comments}

Using the off-shell formulations for 3D $\mathcal{N}$-extended conformal supergravity $[29,30]$ and the results in $[18,19,31,33,68]$, in this paper we have developed the geometric superspace settings to construct arbitrary higher derivative couplings (including $R^{n}$ terms) in supergravity theories with $\mathcal{N} \leq 3$. We have concentrated on the explicit construction of all supersymmetric invariants with up to and including four derivatives, since these invariants are used in the models for $\mathcal{N}=1$ and $\mathcal{N}=2$ massive supergravity advocated in [20, 21, 23].

All four-derivative invariants in $\mathcal{N}=1$ supergravity were constructed in [20,21] using component techniques. However, these papers did not provide tools to generate arbitrary higher order invariants. The novelty of our superspace approach is twofold: (i) our construction is geometric; (ii) it allows one to generate supersymmetric invariants of arbitrary order in powers of curvature and its covariant derivatives.

There are three off-shell formulations for $3 \mathrm{D} \mathcal{N}=2$ Poincaré and AdS supergravity theories [29, 33]: (i) type I minimal; (ii) type II minimal; and (iii) non-minimal. For the minimal $\mathcal{N}=2$ supergravity theories, four-derivative invariants were derived in [23] using the component superconformal tensor calculus. In the present paper, we have developed an alternative approach which is not only geometric but also possesses, unlike the one of [23], the following key properties: (i) it allows the generation of supersymmetric invariants of arbitrary order in powers of the curvature and its covariant derivatives; (ii) it keeps manifest the local superconformal symmetry; and (iii) it does not make use of any gauge choice in deriving the curvature squared invariants. The important point of our constructions is that they provide a complete description of the fourth order invariants for the minimal supergravity theories. In particular, we have described the Ricci curvature squared invariant in type I supergravity beyond the bosonic level originally given in [23]. For the case of type II supergravity we have provided a geometric explanation for the gauge-dependent procedure used in [23] to construct the Ricci curvature squared invariant. We have also given a simple geometric reason for the non-existence of two independent invariants containing $R h^{2}$ terms. Finally, we have provided for the first time a geometric setup to construct arbitrary higher derivative invariants within non-minimal supergravity.

Using the supergravity invariants given in the $\mathcal{N}=1$ and minimal $\mathcal{N}=2$ supergravity cases, we have constructed general supergravity models with curvature-squared and lower order terms in order to study models for massive supergravity. In these cases we have derived the superfield equations of motion, linearised them about maximally supersymmetric backgrounds and obtained restrictions on the parameters that have lead to models for massive supergravity. To derive the superfield equations of motion, we have worked out in appendices $\mathrm{B}$ and $\mathrm{C}$ the response of all geometric objects to an infinitesimal prepotential deformation. For type I supergravity we have identified a new massive supergravity model which does not propagate any degrees of freedom associated with the component field $M$ about any AdS background satisfying (3.65). In the the non-minimal formulation for $\mathcal{N}=2$ supergravity we have constructed for the first time a novel consistent theory of massive supergravity. 
In the case of $\mathcal{N}=3$ supergravity we have considered the off-shell formulation with a compensating vector multiplet. For this supergravity theory the AdS action was given in [29], the off-shell $\mathcal{N}=3$ conformal supergravity action was constructed in [31] and the model for topologically massive supergravity was studied in [19]. In the present paper we have constructed for the first time new higher derivative invariants with four and less derivatives. The new $\mathcal{N}=3$ invariants constructed appear to be analogous to the invariants in the type II case in $\mathcal{N}=2$ supergravity. However, by using the vector multiplet as compensator it does not seem possible to remove one of the torsion components $\mathcal{S}, \mathcal{S}^{I J}$ or $\mathcal{C}_{a}{ }^{I J}$ by gauge fixing. Thus it appears that more invariants are possible in the $\mathcal{N}=3$ case. Perhaps the most interesting point raised in our $\mathcal{N}=3$ analysis is that there appears to exist an independent scalar curvature squared invariant in contrast to the the type II case. Our results provide the building blocks for the construction of general massive $\mathcal{N}=3$ supergravity theories.

The off-shell $\mathcal{N}=3$ formulation with a compensating vector multiplet corresponds to $(2,1)$ AdS supergravity. So far the appropriate compensator for $(3,0)$ AdS supergravity is not known. However, one expects the compensator to be described by a Lorentz and $\mathrm{SO}(3)$ scalar primary superfield $Y$, while without loss of generality we can take this compensator to be have dimension $1, \mathbb{D} Y=Y$. It is expected that $(3,0)$ AdS superspace is a solution to the equations of motion for $\mathcal{N}=3$ supergravity coupled to the compensator. On the other hand one can describe $(3,0)$ AdS superspace by imposing some differential constraints on $Y$. The appropriate constraints are ${ }^{20}$

$$
\begin{aligned}
\nabla^{\gamma(I} \nabla_{\gamma}^{J)} Y & =\frac{1}{3} \delta^{I J} \nabla_{K}^{\gamma} \nabla_{\gamma}^{K} Y, \\
\nabla_{(\alpha}^{[I} \nabla_{\beta)}^{J]} Y^{-1} & =0 .
\end{aligned}
$$

To see this, one just degauges to conventional superspace [30] and imposes the gauge condition $Y=1$. We find the following constraints on the torsion superfields:

$$
\mathcal{S}^{I J}=0, \quad \mathcal{C}_{a}{ }^{I J}=0,
$$

which defines $(3,0)$ AdS superspace [69]. It is also interesting to note that the constraints (7.1) implies the equation

$$
\nabla_{K}^{\gamma} \nabla_{\gamma}^{K} Y^{-\frac{1}{2}}-6 \mathrm{i} \mu Y^{\frac{1}{2}}=0, \quad \mu=\mathrm{const},
$$

where $\mu$ coincides with $\mathcal{S}$ in the gauge $Y=1$. There appears to be a striking similarity between the constraint (7.1a) and the one defining the $\mathcal{N}=2$ off-shell vector multiplet suggesting that perhaps one should treat it as an off-shell condition for the compensator. We thus suggest that the constraint (7.1a) defines an off-shell multiplet while (7.1b) is an on-shell condition derived from an appropriate action which is currently unknown.

\footnotetext{
${ }^{20}$ In the isospinor notation, the constraint (7.1a) can be written in the compact form $\nabla^{\alpha(i j} \nabla_{\alpha}^{k l)} Y=0$. The constraint (7.1b) implies the vanishing of the super-Cotton tensor. In the super-Poincaré case, the constraint (7.1a) describes one of the two $\mathcal{N}=3$ multiplets obtained by reducing the $\mathcal{N}=4$ supercurrent to $\mathcal{N}=3$ Minkowski superspace [73], with the second multiplet being the $\mathcal{N}=3$ supercurrent.
} 
In this paper we have restricted our attention to supergravity with $\mathcal{N} \leq 3$. Keeping in mind certain similarities between the general $\mathcal{N}=3$ and $\mathcal{N}=4$ supergravity-matter systems, see [29] for more details, it is natural to expect that techniques analogous to those used for the $\mathcal{N}=3$ case can be applied to the $\mathcal{N}=4$ case in order to construct higher derivative couplings.

In conclusion, we give a bi-product of our analysis and present superfield equations for massive higher spin multiplets in $(1,0),(1,1)$ and $(2,0)$ anti-de Sitter superspaces. A massive higher spin multiplet in $\mathcal{N}=1$ AdS superspace, eq. (2.60), is described by a real symmetric rank- $n$ spinor, $T_{\alpha_{1} \cdots \alpha_{n}}=T_{\left(\alpha_{1} \cdots \alpha_{n}\right)}$, constrained by

$$
\begin{aligned}
\mathcal{D}^{\beta} T_{\alpha_{1} \cdots \alpha_{n-1} \beta} & =0, \\
\left(\frac{\mathrm{i}}{2} \mathcal{D}^{2}+m\right) T_{\alpha_{1} \cdots \alpha_{n}} & =0,
\end{aligned}
$$

with $m$ a real mass parameter. It can be shown that

$$
\left(\frac{\mathrm{i}}{2} \mathcal{D}^{2}\right)^{2} T_{\alpha_{1} \cdots \alpha_{n}}=\left(\mathcal{D}^{a} \mathcal{D}_{a}-\mathrm{i}(n+2) \mathcal{S} \mathcal{D}^{2}-n(n+2) \mathcal{S}^{2}\right) T_{\alpha_{1} \cdots \alpha_{n}}
$$

where the second term on the right can be rewritten as follows:

$$
\frac{\mathrm{i}}{2} \mathcal{D}^{2} T_{\alpha_{1} \cdots \alpha_{n}}=-\mathcal{D}_{\left(\alpha_{1}\right.}{ }^{\beta} T_{\left.\alpha_{2} \cdots \alpha_{n}\right) \beta}-(n+2) \mathcal{S} T_{\alpha_{1} \cdots \alpha_{n}} .
$$

A massive higher spin multiplet in $(1,1)$ AdS superspace, which corresponds to the algebra (3.62) with $\mathcal{C}_{\alpha \beta}=0$, is described by a real symmetric rank- $n$ spinor, $T_{\alpha_{1} \cdots \alpha_{n}}=$ $T_{\left(\alpha_{1} \cdots \alpha_{n}\right)}$ constrained by

$$
\begin{aligned}
\mathcal{D}^{\beta} T_{\alpha_{1} \cdots \alpha_{n-1} \beta}=\overline{\mathcal{D}}^{\beta} T_{\alpha_{1} \cdots \alpha_{n-1} \beta} & =0, \\
\left(\frac{\mathrm{i}}{2} \mathcal{D}^{\gamma} \overline{\mathcal{D}}_{\gamma}+m\right) T_{\alpha_{1} \cdots \alpha_{n}} & =0 .
\end{aligned}
$$

It can be shown that

$$
\left(\frac{\mathrm{i}}{2} \mathcal{D}^{\gamma} \overline{\mathcal{D}}_{\gamma}\right)^{2} T_{\alpha_{1} \cdots \alpha_{n}}=\left(\mathcal{D}^{a} \mathcal{D}_{a}+2(n+2)|\mathcal{R}|^{2}\right) T_{\alpha_{1} \cdots \alpha_{n}}
$$

In the case of $(2,0)$ AdS superspace, which corresponds to the algebra (4.55) with $\mathcal{C}_{a}=0$, massive higher spin multiplets are also described by the equations (7.7). However, the identity (7.8) turns into

$$
\left(\frac{\mathrm{i}}{2} \mathcal{D}^{\gamma} \overline{\mathcal{D}}_{\gamma}\right)^{2} T_{\alpha_{1} \cdots \alpha_{n}}=\left(\mathcal{D}^{a} \mathcal{D}_{a}+(n+2) \mathrm{i} \mathcal{S} \mathcal{D}^{\gamma} \overline{\mathcal{D}}_{\gamma}-n(n+2) \mathcal{S}^{2}\right) T_{\alpha_{1} \cdots \alpha_{n}}
$$

where the second term on the right can be rewritten as follows:

$$
\frac{\mathrm{i}}{2} \mathcal{D}^{\gamma} \overline{\mathcal{D}}_{\gamma} T_{\alpha_{1} \cdots \alpha_{n}}=\mathcal{D}_{\left(\alpha_{1}\right.}{ }^{\gamma} T_{\left.\alpha_{2} \cdots \alpha_{n}\right) \gamma}+(n+2) \mathcal{S} T_{\alpha_{1} \cdots \alpha_{n}}
$$




\section{Acknowledgments}

This work was supported in part by the Australian Research Council (ARC) Discovery Project DP140103925. The work of GT-M and JN was supported by the ARC Discovery Early Career Award (DECRA), project No. DE120101498. GT-M is grateful to the Instituut voor Theoretische Fysica, KU Leuven, for kind hospitality during January-May 2015. From July 2015 the work of GT-M is supported by the Interuniversity Attraction Poles Programme initiated by the Belgian Science Policy (P7/37) and in part by COST Action MP1210 "The String Theory Universe."

\section{A Geometry of conformal superspace}

Here we collect the essential details of the $\mathcal{N}$-extended superspace geometry developed in [30] for the cases $\mathcal{N}=1,2,3$.

We begin with a curved three-dimensional $\mathcal{N}$-extended superspace $\mathcal{M}^{3 \mid 2 \mathcal{N}}$ parametrized by local bosonic $\left(x^{m}\right)$ and fermionic coordinates $\left(\theta_{I}^{\mu}\right)$ :

$$
z^{M}=\left(x^{m}, \theta_{I}^{\mu}\right),
$$

where $m=0,1,2, \mu=1,2$ and $I=1, \cdots, \mathcal{N}$. The structure group is chosen to be $\operatorname{OSp}(\mathcal{N} \mid 4, \mathbb{R})$ and the covariant derivatives have the form

$$
\nabla_{A}=E_{A}{ }^{M} \partial_{M}-\omega_{A}^{\underline{b}} X_{\underline{b}}=E_{A}^{M} \partial_{M}-\frac{1}{2} \Omega_{A}^{a b} M_{a b}-\frac{1}{2} \Phi_{A}^{P Q} N_{P Q}-B_{A} \mathbb{D}-\mathfrak{F}_{A}^{B} K_{B} .
$$

Here $E_{A}=E_{A}^{M}(z) \partial_{M}$ is the inverse supervielbein, $M_{a b}$ are the Lorentz generators, $N_{I J}$ are generators of the $\mathrm{SO}(\mathcal{N})$ group, $\mathbb{D}$ is the dilatation generator and $K_{A}=\left(K_{a}, S_{\alpha}^{I}\right)$ are the special superconformal generators. ${ }^{21}$ The supervielbein $E^{A}=\mathrm{d} z^{M} E_{M}{ }^{A}$ is defined such that

$$
E_{M}^{A} E_{A}^{N}=\delta_{M}^{N}, \quad E_{A}^{M} E_{M}^{B}=\delta_{A}^{B} .
$$

The Lorentz generators obey

$$
\begin{aligned}
{\left[M_{a b}, M_{c d}\right] } & =2 \eta_{c[a} M_{b] d}-2 \eta_{d[a} M_{b] c}, \\
{\left[M_{a b}, \nabla_{c}\right] } & =2 \eta_{c[a} \nabla_{b]}, \quad\left[M_{\alpha \beta}, \nabla_{\gamma}^{I}\right]=\varepsilon_{\gamma(\alpha} \nabla_{\beta)}^{I} .
\end{aligned}
$$

The $\mathrm{SO}(\mathcal{N})$ and dilatation generators obey

$$
\begin{aligned}
{\left[N_{K L}, N^{I J}\right] } & =2 \delta_{[K}^{I} N_{L]}^{J}-2 \delta_{[K}^{J} N_{L]}^{I}, & {\left[N_{K L}, \nabla_{\alpha}^{I}\right] } & =2 \delta_{[K}^{I} \nabla_{\alpha L]}, \\
{\left[\mathbb{D}, \nabla_{a}\right] } & =\nabla_{a}, & {\left[\mathbb{D}, \nabla_{\alpha}^{I}\right] } & =\nabla_{\alpha}^{I} .
\end{aligned}
$$

The special conformal generators $K_{A}$ transform under Lorentz and $\mathrm{SO}(\mathcal{N})$ transformations as

$$
\left[M_{a b}, K_{c}\right]=2 \eta_{c[a} K_{b]}, \quad\left[M_{\alpha \beta}, S_{\gamma}^{I}\right]=\varepsilon_{\gamma(\alpha} S_{\beta)}^{I}, \quad\left[N_{K L}, S_{\alpha}^{I}\right]=2 \delta_{[K}^{I} S_{\alpha L]},
$$

\footnotetext{
${ }^{21}$ As usual, we refer to $K_{a}$ as the special conformal generator and $S_{\alpha}^{I}$ as the $S$-supersymmetry generator.
} 
while under dilatations as

$$
\left[\mathbb{D}, K_{a}\right]=-K_{a}, \quad\left[\mathbb{D}, S_{\alpha}^{I}\right]=-\frac{1}{2} S_{\alpha}^{I} .
$$

Among themselves, the generators $K_{A}$ obey the algebra

$$
\left\{S_{\alpha}^{I}, S_{\beta}^{J}\right\}=2 \mathrm{i} \delta^{I J}\left(\gamma^{c}\right)_{\alpha \beta} K_{c} .
$$

Finally, the algebra of $K_{A}$ with $\nabla_{A}$ is given by

$$
\begin{aligned}
{\left[K_{a}, \nabla_{b}\right] } & =2 \eta_{a b} \mathbb{D}+2 M_{a b}, \\
{\left[K_{a}, \nabla_{\alpha}^{I}\right] } & =-\mathrm{i}\left(\gamma_{a}\right)_{\alpha}{ }^{\beta} S_{\beta}^{I}, \\
{\left[S_{\alpha}^{I}, \nabla_{a}\right] } & =\mathrm{i}\left(\gamma_{a}\right)_{\alpha}{ }^{\beta} \nabla_{\beta}^{I}, \\
\left\{S_{\alpha}^{I}, \nabla_{\beta}^{J}\right\} & =2 \varepsilon_{\alpha \beta} \delta^{I J} \mathbb{D}-2 \delta^{I J} M_{\alpha \beta}-2 \varepsilon_{\alpha \beta} N^{I J} .
\end{aligned}
$$

All other (anti-)commutators vanish.

The covariant derivatives obey the (anti-)commutation relations of the form

$$
\begin{aligned}
{\left[\nabla_{A}, \nabla_{B}\right\}=} & -T_{A B}{ }^{C} \nabla_{C}-\frac{1}{2} R(M)_{A B}{ }^{c d} M_{c d}-\frac{1}{2} R(N)_{A B}{ }^{P Q} N_{P Q} \\
& -R(\mathbb{D})_{A B} \mathbb{D}-R(S)_{A B}{ }_{K}^{\gamma} S_{\gamma}^{K}-R(K)_{A B}{ }^{c} K_{c}
\end{aligned}
$$

where $T$ is the torsion, and $R(M), R(N), R(\mathbb{D}), R(K)$ are the curvatures.

The covariant derivatives transform under the conformal supergravity gauge group as follows

$$
\delta_{\mathcal{G}} \nabla_{A}=\left[\mathcal{K}, \nabla_{A}\right],
$$

where $\mathcal{K}$ denotes the first-order differential operator

$$
\mathcal{K}=\xi^{C} \nabla_{C}+\frac{1}{2} \Lambda^{a b} M_{a b}+\frac{1}{2} \Lambda^{I J} N_{I J}+\sigma \mathbb{D}+\Lambda^{A} K_{A} .
$$

Covariant (or tensor) superfields transform as

$$
\delta_{\mathcal{G}} T=\mathcal{K} T
$$

The algebra of covariant derivatives are constrained entirely in terms of a single primary superfield, the super-Cotton tensor. It is used in the construction of the component fields of the 3D Weyl multiplet [31]. The super-Cotton tensor takes different forms for the $\mathcal{N}=1,2,3$ cases. We summarise these cases below.

\section{A.1 The $\mathcal{N}=1$ case}

The $\mathcal{N}=1$ super-Cotton tensor $W_{\alpha \beta \gamma}$ is a symmetric primary superfield of dimension-5/2

$$
S_{\delta} W_{\alpha \beta \gamma}=0, \quad \mathbb{D} W_{\alpha \beta \gamma}=\frac{5}{2} W_{\alpha \beta \gamma} .
$$


The algebra of covariant derivatives is given by

$$
\begin{aligned}
\left\{\nabla_{\alpha}, \nabla_{\beta}\right\} & =2 \mathrm{i} \nabla_{\alpha \beta}, \\
{\left[\nabla_{a}, \nabla_{\alpha}\right] } & =\frac{1}{4}\left(\gamma_{a}\right)_{\alpha}{ }^{\beta} W_{\beta \gamma \delta} K^{\gamma \delta}, \\
{\left[\nabla_{a}, \nabla_{b}\right] } & =-\frac{\mathrm{i}}{8} \varepsilon_{a b c}\left(\gamma^{c}\right)^{\alpha \beta} \nabla_{\alpha} W_{\beta \gamma \delta} K^{\gamma \delta}-\frac{1}{4} \varepsilon_{a b c}\left(\gamma^{c}\right)^{\alpha \beta} W_{\alpha \beta \gamma} S^{\gamma},
\end{aligned}
$$

The Bianchi identities imply an additional constraint on $W_{\alpha \beta \gamma}$,

$$
\nabla^{\alpha} W_{\alpha \beta \gamma}=0
$$

\section{A.2 The $\mathcal{N}=2$ case}

Here we make use of the complex basis for the $\mathcal{N}=2$ covariant derivatives $\nabla_{A}=$ $\left(\nabla_{\alpha}, \bar{\nabla}^{\alpha}, \nabla_{a}\right)$, see [30] for more details. The complex spinor covariant derivatives have definite $\mathrm{U}(1)$ charges:

$$
\left[J, \nabla_{\alpha}\right]=\nabla_{\alpha}, \quad\left[J, \bar{\nabla}_{\alpha}\right]=-\bar{\nabla}_{\alpha},
$$

with the $\mathrm{U}(1)$ generator defined by

$$
J:=-\frac{\mathrm{i}}{2} \varepsilon^{K L} N_{K L}
$$

The $\mathrm{SO}(2)$ connection and curvature take the form

$$
\frac{1}{2} \Phi_{A}{ }^{K L} N_{K L}=\mathrm{i} \Phi_{A} J, \quad \frac{1}{2} R(N)_{A B}{ }^{K L} N_{K L}=\mathrm{i} R(J)_{A B} J .
$$

The conjugation rule in the complex basis is

$$
\left(\nabla_{\alpha} F\right)^{*}=(-1)^{\varepsilon(F)} \bar{\nabla}_{\alpha} \bar{F},
$$

where $F$ is a complex superfield and $\bar{F}=(F)^{*}$ is its complex conjugate.

The super-Cotton tensor $W_{\alpha \beta}$ is a symmetric primary superfield of dimension 2

$$
S_{\gamma} W_{\alpha \beta}=0, \quad \mathbb{D} W_{\alpha \beta}=2 W_{\alpha \beta} .
$$

As in the $\mathcal{N}=1$ case, its spinor divergence vanishes,

$$
\nabla^{\alpha} W_{\alpha \beta}=0 .
$$

In the complex basis $\left(\nabla_{\alpha}, \bar{\nabla}_{\alpha}\right)$, the covariant derivative algebra takes the form

$$
\begin{aligned}
\left\{\nabla_{\alpha}, \nabla_{\beta}\right\} & =0 \\
\left\{\nabla_{\alpha}, \bar{\nabla}_{\beta}\right\}= & -2 \mathrm{i} \nabla_{\alpha \beta}-\varepsilon_{\alpha \beta} W_{\gamma \delta} K^{\gamma \delta}, \\
{\left[\nabla_{a}, \nabla_{\beta}\right]=} & \frac{\mathrm{i}}{2}\left(\gamma_{a}\right)_{\beta}^{\gamma} \nabla_{\gamma} W^{\alpha \delta} K_{\alpha \delta}-\left(\gamma_{a}\right)_{\beta \gamma} W^{\gamma \delta} \bar{S}_{\delta}, \\
{\left[\nabla_{a}, \nabla_{b}\right]=} & -\frac{\mathrm{i}}{8} \varepsilon_{a b c}\left(\gamma^{c}\right)^{\gamma \delta}\left(\mathrm{i}\left[\nabla_{\gamma}, \bar{\nabla}_{\delta}\right] W_{\alpha \beta} K^{\alpha \beta}+4 \bar{\nabla}_{\gamma} W_{\delta \beta} \bar{S}^{\beta}+4 \nabla_{\gamma} W_{\delta \beta} S^{\beta}\right. \\
& \left.-8 W_{\gamma \delta} J\right) .
\end{aligned}
$$


Here the generators $M_{a b}, J, \mathbb{D}, S_{\alpha}, \bar{S}^{\alpha}, K_{a}$ and the covariant derivatives $\nabla_{A}$ satisfy the following algebraic relations

$$
\begin{aligned}
& {\left[M_{\alpha \beta}, \nabla_{\gamma}\right]=\varepsilon_{\gamma(\alpha} \nabla_{\beta}, \quad\left[\mathbb{D}, \nabla_{\alpha}\right]=\frac{1}{2} \nabla_{\alpha},} \\
& \left\{S_{\alpha}, S_{\beta}\right\}=0, \quad\left\{S_{\alpha}, \bar{S}_{\beta}\right\}=2 \mathrm{i} K_{\alpha \beta}, \\
& {\left[S_{\alpha}, K_{b}\right]=0 \text {, }} \\
& {\left[M_{\alpha \beta}, S_{\gamma}\right]=\varepsilon_{\gamma(\alpha} S_{\beta)}, \quad\left[J, S_{\alpha}\right]=-S_{\alpha}, \quad\left[\mathbb{D}, S_{\alpha}\right]=-\frac{1}{2} S_{\alpha},} \\
& {\left[K_{a}, \nabla_{\alpha}\right]=-\mathrm{i}\left(\gamma_{a}\right)_{\alpha}{ }^{\beta} \bar{S}_{\beta}, \quad\left[S_{\alpha}, \nabla_{a}\right]=-\mathrm{i}\left(\gamma_{a}\right)_{\alpha}{ }^{\beta} \bar{\nabla}_{\beta},} \\
& \left\{\bar{S}_{\alpha}, \nabla_{\beta}\right\}=0, \quad\left\{S_{\alpha}, \nabla_{\beta}\right\}=2 \varepsilon_{\alpha \beta} \mathbb{D}-2 M_{\alpha \beta}-2 \varepsilon_{\alpha \beta} J,
\end{aligned}
$$

together with their complex conjugates.

\section{A.3 The $\mathcal{N}=3$ case}

The $\mathcal{N}=3$ super-Cotton tensor $W_{\alpha}$ is a primary superfield of dimension $3 / 2$ with vanishing spinor divergence,

$$
S_{\beta}^{I} W_{\alpha}=0, \quad \mathbb{D} W_{\alpha}=\frac{3}{2} W_{\alpha}, \quad \nabla^{\alpha I} W_{\alpha}=0 .
$$

The algebra of covariant derivatives is

$$
\begin{aligned}
\left\{\nabla_{\alpha}^{I}, \nabla_{\beta}^{J}\right\}= & 2 \mathrm{i} \delta^{I J} \nabla_{\alpha \beta}-2 \varepsilon_{\alpha \beta} \varepsilon^{I J L} W^{\gamma} S_{\gamma L}+\mathrm{i} \varepsilon_{\alpha \beta}\left(\gamma^{c}\right)^{\gamma \delta} \varepsilon^{I J K}\left(\nabla_{\gamma K} W_{\delta}\right) K_{c}, \\
{\left[\nabla_{a}, \nabla_{\beta}^{J}\right]=} & \mathrm{i} \varepsilon^{J K L}\left(\gamma_{a}\right)_{\beta \gamma} W^{\gamma} N_{K L}+\mathrm{i} \varepsilon^{J K L}\left(\gamma_{a}\right)_{\beta \gamma}\left(\nabla_{K}^{\gamma} W^{\delta}\right) S_{\delta L} \\
& +\frac{1}{4} \varepsilon^{J K L}\left(\gamma_{a}\right)_{\beta \gamma}\left(\gamma^{c}\right)_{\delta \rho}\left(\nabla_{K}^{\gamma} \nabla_{L}^{\delta} W^{\rho}\right) K_{c}, \\
{\left[\nabla_{a}, \nabla_{b}\right]=} & \varepsilon_{a b c}\left(\gamma^{c}\right)_{\alpha \beta}\left[-\frac{1}{2} \varepsilon^{I J K}\left(\nabla_{I}^{\alpha} W^{\beta}\right) N_{J K}-\frac{1}{4} \varepsilon^{I J K}\left(\nabla_{I}^{\alpha} \nabla_{J}^{\beta} W^{\gamma}\right) S_{\gamma K}\right. \\
+ & \left.\frac{\mathrm{i}}{24} \varepsilon^{I J K}\left(\gamma^{d}\right)_{\gamma \delta}\left(\nabla_{I}^{\alpha} \nabla_{J}^{\beta} \nabla_{K}^{\gamma} W^{\delta}\right) K_{d}\right] .
\end{aligned}
$$

\section{B Results for $\mathcal{N}=1$ prepotential deformation}

In order to compute the equations of motion corresponding to the supergravity action (2.52), it is necessary to know how the functionals listed in subsection 2.3 depend on the unconstrained prepotential for $\mathcal{N}=1$ conformal supergravity, which is a real symmetric rank-3 spinor $\Psi_{\alpha \beta \gamma}$ in accordance with the prepotential formulation for $3 \mathrm{D} \mathcal{N}=1$ supergravity sketched in [26]. Here we will build on the ideas put forward in the classic papers by Grisaru and Siegel $[74,75]$ devoted to the background field method in 4D $\mathcal{N}=1$ supergravity (see [45] for a pedagogical review and applications).

In $\mathcal{N}=1$ conformal supergravity, the gauge group consists of (i) the super-Weyl transformations (2.7); and (ii) the superspace general coordinate and local Lorentz transformations, which have the infinitesimal form

$$
\delta_{\mathcal{K}} \mathcal{D}_{A}=\left[\mathcal{K}, \mathcal{D}_{A}\right], \quad \mathcal{K}=\xi^{B}(z) \mathcal{D}_{B}+\frac{1}{2} K^{b c}(z) M_{b c},
$$

with the gauge parameters $\xi^{B}$ and $K^{b c}$ obeying natural reality conditions but otherwise arbitrary. 
Let $\mathbb{D}_{A}$ be some other set of covariant derivatives which differ from $\mathcal{D}_{A}$ by finite deformations but satisfy the same (anti-)commutation relations (2.4) as the covariant derivatives $\mathcal{D}_{A}$. We can represent

$$
\mathbb{D}_{A}=\mathbb{E}_{A}^{B} \mathcal{D}_{B}-\frac{1}{2} \boldsymbol{\Omega}_{A}^{b c} M_{b c}
$$

for some tensor superfields $\mathbb{E}_{A}^{B}$ and $\Omega_{A}^{b c}$. In such a setting, the gauge transformations (2.7) and (B.1) can be realised in two different incarnations: (i) as "background" transformations; and (ii) as "quantum" transformations [74, 75]. The latter gauge freedom associated with super-Weyl $(\sigma)$, coordinate $\left(\xi^{\beta}\right)$ and local Lorentz $\left(K^{b c}\right)$ parameters may be used to bring the operator $\mathbb{D}_{\alpha}$ to the form:

$$
\mathbb{D}_{\alpha}=\mathcal{D}_{\alpha}+\mathrm{i} \Psi_{\alpha \gamma \delta} \mathcal{D}^{\gamma \delta}-\frac{1}{2} \boldsymbol{\Omega}_{\alpha}^{\gamma \delta} M_{\gamma \delta}, \quad \Psi_{\alpha \beta \gamma}=\Psi_{(\alpha \beta \gamma)} .
$$

The deformed connection $\boldsymbol{\Omega}_{\alpha}{ }^{\gamma \delta}$ may be determined as a function of the prepotential $\Psi_{\alpha \beta \gamma}$ by requiring the spinor derivatives $\mathbb{D}_{\alpha}$ to satisfy the same algebra as that of conventional superspace (2.4). Specifically, we require

$$
\begin{aligned}
\left\{\mathbb{D}_{\alpha}, \mathbb{D}_{\beta}\right\}= & 2 \mathrm{i} \mathbb{D}_{\alpha \beta}-4 \mathrm{i} \mathbb{S} M_{\alpha \beta}, \\
{\left[\mathbb{D}_{\alpha \beta}, \mathbb{D}_{\gamma}\right]=} & -2 \varepsilon_{\gamma(\alpha} \mathbb{S D}_{\beta)}+2 \varepsilon_{\gamma(\alpha} \mathbb{C}_{\beta) \delta \rho} M^{\delta \rho} \\
& +\frac{2}{3}\left(\mathcal{D}_{\gamma} \mathbb{S} M_{\alpha \beta}-4 \mathcal{D}_{(\alpha} \mathbb{S} M_{\beta) \gamma}\right),
\end{aligned}
$$

where we define the deformed vector derivative by

$$
\mathbb{D}_{a}=\frac{\mathrm{i}}{4}\left(\gamma_{a}\right)^{\alpha \beta}\left\{\mathbb{D}_{\alpha}, \mathbb{D}_{\beta}\right\}+2 \mathbb{S} M_{a} .
$$

The above torsion superfields $\mathbb{C}_{a}$ and $\mathbb{S}$ are some functions of the prepotential $\Psi_{\alpha \beta \gamma}$ and its covariant derivatives.

Requiring the algebra (B.4) fixes $\boldsymbol{\Omega}_{\alpha}^{\gamma \delta}$ as a function of $\Psi_{\alpha \beta \gamma}$ and its covariant derivatives. The deformed spinor covariant derivative is

$$
\begin{aligned}
\mathbb{D}_{\alpha}= & \mathcal{D}_{\alpha}+\mathrm{i} \Psi_{\alpha \gamma \delta} \mathcal{D}^{\gamma \delta}-\frac{1}{4} \mathcal{D}^{2} \Psi_{\alpha \beta \gamma} M^{\beta \gamma}-\frac{\mathrm{i}}{2} \mathcal{D}_{(\alpha}{ }^{\delta} \Psi_{\beta \gamma) \delta} M^{\beta \gamma} \\
& -\frac{2 \mathrm{i}}{3} \mathcal{D}_{\beta \gamma} \Psi^{\beta \gamma \delta} M_{\delta \alpha}+\frac{3 \mathrm{i}}{2} \mathcal{S} \Psi_{\alpha \beta \gamma} M^{\beta \gamma}+\mathcal{O}\left(\Psi^{2}\right) .
\end{aligned}
$$

Here we have omitted all the terms of second and higher order in $\Psi_{\alpha \beta \gamma}$, for these terms are not necessary for our goals in the present paper. All the results below also hold modulo terms quadratic in $\Psi_{\alpha \beta \gamma}$, but we do not write explicitly $\mathcal{O}\left(\Psi^{2}\right)$. Since $\mathbb{D}_{\alpha}$ has been determined, requiring the (anti-)commutation relations (B.4) fixes the torsion superfields as follows:

$$
\begin{aligned}
\mathbb{S}= & \mathcal{S}-\frac{1}{8}\left(\mathcal{D}^{(\alpha} \mathcal{D}^{\beta \gamma)}+2 \mathcal{C}^{\alpha \beta \gamma}\right) \Psi_{\alpha \beta \gamma} \\
\mathbb{C}_{\alpha \beta \gamma}= & \mathcal{C}_{\alpha \beta \gamma}+\frac{1}{4} \mathcal{D}_{(\alpha}{ }^{\delta} \mathcal{D}^{2} \Psi_{\beta \gamma) \delta}+\frac{\mathrm{i}}{2} \mathcal{D}_{(\alpha}{ }^{\delta} \mathcal{D}_{\beta}{ }^{\rho} \Psi_{\gamma) \delta \rho}-\frac{1}{4} \mathcal{S} \mathcal{D}^{2} \Psi_{\alpha \beta \gamma} \\
& -\frac{5}{3} \mathcal{C}_{(\alpha}{ }^{\rho \tau} \mathcal{D}_{\beta} \Psi_{\gamma \rho \tau)}-\frac{4}{3}\left(\mathcal{D}^{\delta} \mathcal{S}\right) \mathcal{D}_{(\delta} \Psi_{\alpha \beta \gamma)}+\frac{1}{2} \mathcal{C}_{(\alpha \beta}{ }^{\rho} \mathcal{D}^{\tau} \Psi_{\gamma) \rho \tau} \\
& +\frac{1}{3}\left(\mathcal{D}_{(\alpha} \mathcal{S}\right) \mathcal{D}^{\tau} \Psi_{\beta \gamma) \tau}+\frac{\mathrm{i}}{2}\left(\mathcal{D}_{(\alpha}{ }^{\rho} \mathcal{S}\right) \Psi_{\beta \gamma) \rho}-\frac{\mathrm{i}}{2} \mathcal{S}^{2} \Psi_{\alpha \beta \gamma}
\end{aligned}
$$


Finally, the deformed vector covariant derivative is given by

$$
\begin{aligned}
\mathbb{D}_{\alpha \beta}= & \mathcal{D}_{\alpha \beta}+\mathcal{D}_{(\alpha} \Psi_{\beta \gamma \delta)} \mathcal{D}^{\gamma \delta}+\frac{1}{2} \mathcal{D}^{\delta} \Psi_{\delta \rho(\alpha} \mathcal{D}_{\beta)}{ }^{\rho} \\
+ & {\left[-\frac{\mathrm{i}}{4} \mathcal{D}^{2} \Psi_{\alpha \beta \gamma}+\frac{1}{2} \mathcal{D}_{(\alpha}{ }^{\delta} \Psi_{\beta \gamma) \delta}-\frac{1}{3} \mathcal{D}^{\rho \delta} \Psi_{\rho \delta(\alpha} \varepsilon_{\beta) \gamma}+\frac{1}{2} \mathcal{S} \Psi_{\alpha \beta \gamma}\right] \mathcal{D}^{\gamma} } \\
+ & {\left[-\frac{5}{4} \mathcal{D}_{(\alpha}{ }^{\rho} \mathcal{D}_{\beta} \Psi_{\gamma \delta \rho)}+\frac{1}{4} \mathcal{D}_{(\alpha \beta} \mathcal{D}^{\tau} \Psi_{\gamma \delta) \tau}+\frac{1}{4} \mathcal{D}^{\rho \tau}\left(\mathcal{D}_{(\tau} \Psi_{\gamma \rho \alpha)} \varepsilon_{\beta \delta}+\mathcal{D}_{(\tau} \Psi_{\gamma \rho \beta)} \varepsilon_{\alpha \delta}\right)\right.} \\
& -\frac{1}{12} \varepsilon_{\gamma(\alpha} \varepsilon_{\beta) \delta} \mathcal{D}^{\rho \epsilon} \mathcal{D}^{\tau} \Psi_{\rho \tau \epsilon}+\frac{1}{8}\left(\mathcal{D}_{(\alpha}{ }^{\rho} \varepsilon_{\beta)(\gamma} \mathcal{D}^{\tau} \Psi_{\delta) \rho \tau}-\mathcal{D}_{(\gamma}{ }^{\rho} \varepsilon_{\delta)(\alpha} \mathcal{D}^{\tau} \Psi_{\beta) \rho \tau}\right) \\
& -\mathcal{C}_{(\alpha \beta}{ }^{\tau} \Psi_{\gamma \delta) \tau}+\frac{1}{2} \mathcal{C}^{\rho \tau}{ }_{(\alpha} \varepsilon_{\beta)(\gamma} \Psi_{\delta) \rho \tau}-\frac{1}{2} \mathcal{C}^{\rho \tau}{ }_{(\gamma} \varepsilon_{\delta)(\alpha} \Psi_{\beta) \rho \tau}-\frac{1}{6} \varepsilon_{\gamma(\alpha} \varepsilon_{\beta) \delta} \mathcal{C}^{\rho \tau \epsilon} \Psi_{\rho \tau \epsilon} \\
& \left.\left.+\frac{4}{3}\left(\mathcal{D}_{(\alpha} \mathcal{S}\right) \Psi_{\beta \gamma \delta)}-\frac{2}{3}\left(\mathcal{D}^{\rho} \mathcal{S}\right) \Psi_{\rho \gamma(\alpha} \varepsilon_{\beta) \delta}\right)\right] M^{\gamma \delta}
\end{aligned}
$$

For the derivation of the above results and the relations $(2.54 \mathrm{c})$, a number of identities prove to be useful. The most important identities are:

$$
\begin{aligned}
& \mathcal{D}^{2} \mathcal{C}_{\alpha \beta \gamma}=2 \mathrm{i} \mathcal{D}_{(\alpha}{ }^{\delta} \mathcal{C}_{\beta \gamma) \delta}+\frac{8 \mathrm{i}}{3} \mathcal{D}_{(\alpha \beta} \mathcal{D}_{\gamma)} \mathcal{S}+10 \mathrm{i} \mathcal{S} \mathcal{C}_{\alpha \beta \gamma}, \\
& \mathcal{D}^{\beta \gamma} \mathcal{C}_{\alpha \beta \gamma}=-\frac{4}{3} \mathcal{D}_{\alpha \beta} \mathcal{D}^{\beta} \mathcal{S}-4 \mathcal{S} \mathcal{D}_{\alpha} \mathcal{S} \\
& \mathcal{D}_{\alpha} \mathcal{D}^{2}=-2 \mathrm{i} \mathcal{D}_{\alpha \beta} \mathcal{D}^{\beta}+2 \mathrm{i} \mathcal{S} \mathcal{D}_{\alpha}+4 \mathrm{i} \mathcal{S} \mathcal{D}^{\beta} M_{\alpha \beta}+2 \mathrm{i} \mathcal{C}_{\alpha \rho \tau} M^{\rho \tau} \\
& +\frac{8 \mathrm{i}}{3}\left(\mathcal{D}^{\delta} \mathcal{S}\right) M_{\alpha \delta} \\
& \mathcal{D}_{(\alpha}{ }^{\delta} \mathcal{D}_{\beta}{ }^{\rho} \mathcal{C}_{\gamma) \delta \rho}=\mathcal{D}^{a} \mathcal{D}_{a} \mathcal{C}_{\alpha \beta \gamma}-\frac{4}{3} \mathcal{D}_{(\alpha \beta} \mathcal{D}_{\gamma \delta)} \mathcal{D}^{\delta} \mathcal{S}+2 i \mathcal{C}_{\alpha \beta \gamma} \mathcal{D}^{2} \mathcal{S}-6\left(\mathcal{D}_{(\alpha \beta} \mathcal{S}\right) \mathcal{D}_{\gamma)} \mathcal{S} \\
& -4 \mathcal{S D}_{(\alpha \beta} \mathcal{D}_{\gamma)} \mathcal{S}+3 K_{\delta \rho(\alpha \beta} \mathcal{C}_{\gamma)}{ }^{\delta \rho}+10 \mathcal{S}^{2} \mathcal{C}_{\alpha \beta \gamma}, \\
& {\left[\mathcal{D}_{(\alpha}{ }^{\rho}, \mathcal{D}^{2}\right] \mathcal{C}_{\beta \gamma) \rho}=12 \mathrm{i} \mathcal{C}_{\rho(\alpha \beta} \mathcal{D}^{\rho}{ }_{\gamma)} \mathcal{S}-\frac{10 \mathrm{i}}{3}\left(\mathcal{D}^{\delta} \mathcal{S}\right) K_{\alpha \beta \gamma \delta}+\frac{10 \mathrm{i}}{3}\left(\mathcal{D}_{(\alpha} \mathcal{S}\right) \mathcal{D}_{\beta \gamma)} \mathcal{S}} \\
& +\frac{5}{3}\left(\mathcal{D}^{2} \mathcal{S}\right) \mathcal{C}_{\alpha \beta \gamma}, \\
& \varepsilon^{a b c}\left(\gamma_{c}\right)_{(\alpha \beta}\left[\mathcal{D}_{a}, \mathcal{D}_{b}\right] \mathcal{D}_{\gamma)} \mathcal{S}=-\mathrm{i} \mathcal{C}_{\alpha \beta \gamma} \mathcal{D}^{2} \mathcal{S}-2 \mathcal{C}_{(\alpha \beta}{ }^{\rho} \mathcal{D}_{\gamma) \rho} \mathcal{S}+\frac{8}{3}\left(\mathcal{D}_{(\alpha} \mathcal{S}\right) \mathcal{D}_{\beta \gamma)} \mathcal{S} \\
& -2 K_{\alpha \beta \gamma \delta} \mathcal{D}^{\delta} \mathcal{S}, \\
& \varepsilon^{a b c}\left(\gamma_{c}\right)_{(\alpha}{ }^{\delta}\left[\mathcal{D}_{a}, \mathcal{D}_{b}\right] \mathcal{C}_{\beta \gamma) \delta}=6 K_{\delta \rho(\alpha \beta} \mathcal{C}_{\gamma)}{ }^{\delta \rho}-\frac{4}{3}\left(\mathcal{D}^{\delta} \mathcal{S}\right) K_{\alpha \beta \gamma \delta}-\frac{20}{9}\left(\mathcal{D}_{(\alpha} \mathcal{S}\right) \mathcal{D}_{\beta \gamma)} \mathcal{S} \\
& -\frac{4}{3} \mathcal{C}_{(\alpha \beta}{ }^{\delta} \mathcal{D}_{\gamma) \delta} \mathcal{S}+\frac{10 \mathrm{i}}{3}\left(\mathcal{D}^{2} \mathcal{S}\right) \mathcal{C}_{\alpha \beta \gamma}+20 \mathcal{S}^{2} \mathcal{C}_{\alpha \beta \gamma},
\end{aligned}
$$

where we have denoted $K_{\alpha \beta \gamma \delta}:=\mathrm{i} \mathcal{D}_{(\alpha} \mathcal{C}_{\beta \gamma \delta)}$.

In the gauge (B.3), there remains some residual gauge freedom. It is described by certain transformations of the form

$$
\delta \mathbb{D}_{A}:=\left[\mathcal{K}, \mathbb{D}_{A}\right]+\delta_{\sigma} \mathbb{D}_{A}, \quad \mathcal{K}=\xi^{b} \mathbb{D}_{b}+\xi^{\alpha} \mathbb{D}_{\alpha}+\frac{1}{2} K^{b c} M_{b c},
$$

where $\delta_{\sigma} \mathbb{D}_{A}$ denotes an infinitesimal super-Weyl transformation parametrised by $\sigma$, which is obtained from eq. (2.7) by replacing $\mathcal{D}_{A} \rightarrow \mathbb{D}_{A}$. In order to preserve the condition (B.3), 
it may be shown that the parameters $\xi^{\alpha}, K^{b c}$ and $\sigma$ should be functions of $\xi^{a}$ and its covariant derivatives, with $\xi^{a}$ being real unconstrained. Modulo $\Psi$-dependent terms, the parameters $\xi^{\alpha}, K^{b c}$ and $\sigma$ have the explicit form

$$
\begin{aligned}
\xi_{\alpha} & =-\frac{\mathrm{i}}{6} \mathcal{D}^{\beta} \xi_{\beta \alpha}, \\
K_{\alpha \beta} & =2 \mathcal{D}_{(\alpha} \xi_{\beta)}-2 \mathcal{S} \xi_{\alpha \beta}, \\
\sigma & =\mathcal{D}_{\alpha} \xi^{\alpha}
\end{aligned}
$$

The gauge transformation of the prepotential is

$$
\delta \Psi_{\alpha \beta \gamma}=\frac{1}{2} \mathcal{D}_{(\alpha} \xi_{\beta \gamma)}+\mathcal{O}(\Psi) .
$$

Let $S=S\left[\mathcal{D}_{A}, \varphi\right]$ be a supergravity action such as (2.52), with $\varphi$ being the compensator. The action has to be invariant under the supergravity gauge transformations (2.7) and (B.1). Assuming that the compensator obeys its equation of motion, $\delta S / \delta \varphi=0$, we consider the variation of the action induced by an infinitesimal deformation of the gravitational superfield $\Psi_{\alpha \beta \gamma}$,

$$
\delta S\left[\mathcal{D}_{A}, \varphi\right]=\mathrm{i} \int \mathrm{d}^{3 \mid 2} z E \delta \Psi^{\alpha \beta \gamma} \mathbb{T}_{\alpha \beta \gamma},
$$

for some superfield $\mathbb{T}_{\alpha \beta \gamma}$. This variation must vanish if $\delta \Psi^{\alpha \beta \gamma}$ is the gauge transformation (B.12). Since the gauge parameter $\xi_{\beta \gamma}$ in (B.12) is an arbitrary superfield, we conclude that

$$
\mathcal{D}^{\gamma} \mathbb{T}_{\alpha \beta \gamma}=0
$$

\section{Results for $\mathcal{N}=2$ prepotential deformation}

The prepotential formulation for $\mathcal{N}=2$ conformal supergravity was given in [68] as a generalisation of the prepotential solution in $4 \mathrm{D} \mathcal{N}=1$ supergravity [76]. Modulo purely gauge degrees of freedom, the $3 \mathrm{D} \mathcal{N}=2$ Weyl multiplet is described by a real vector superfield $H^{a}$. In order to derive the equations of motion for the supergravity actions (3.52) and (4.45), we have to know the dependence of these actions on $H^{a}$. The necessary technical tools are given in this appendix.

In $\mathcal{N}=2$ conformal supergravity, the gauge group consists of (i) the super-Weyl transformations (3.5); and (ii) the superspace general coordinate and local Lorentz and $\mathrm{U}(1)_{R}$ transformations, which have the infinitesimal form

$$
\delta_{\mathcal{K}} \mathcal{D}_{A}=\left[\mathcal{K}, \mathcal{D}_{A}\right], \quad \mathcal{K}=\xi^{B}(z) \mathcal{D}_{B}+\frac{1}{2} K^{b c}(z) M_{b c}+\mathrm{i} \tau(z) J
$$

where the gauge parameters $\xi^{B}, K^{b c}$ and $\tau$ obey natural reality conditions but are otherwise arbitrary.

Let $\mathbb{D}_{A}$ be some other set of covariant derivatives which differ from $\mathcal{D}_{A}$ by finite deformations but satisfy the same (anti-)commutation relations (3.3) as the covariant derivatives 
$\mathcal{D}_{A}$. By analogy with background-quantum splitting in $4 \mathrm{D} \mathcal{N}=1$ supergravity $[45,74,75]$, the operators $\mathbb{D}_{\alpha}$ and $\overline{\mathbb{D}}_{\alpha}$ may be represented in the form

$$
\begin{aligned}
& \mathbb{D}_{\alpha}=\mathrm{e}^{\mathcal{W}}\left[\mathcal{F} \mathcal{D}_{\alpha}-\frac{1}{2} \Delta \Omega_{\alpha}{ }^{b c} M_{b c}-\mathrm{i} \Delta \Phi_{\alpha} J\right] \mathrm{e}^{-\mathcal{W}}, \\
& \overline{\mathbb{D}}_{\alpha}=\mathrm{e}^{\overline{\mathcal{W}}}\left[\overline{\mathcal{F}} \mathcal{D}_{\alpha}-\frac{1}{2} \Delta \bar{\Omega}_{\alpha}^{b c} M_{b c}-\mathrm{i} \Delta \bar{\Phi}_{\alpha} J\right] \mathrm{e}^{-\overline{\mathcal{W}}},
\end{aligned}
$$

for some complex first-order operator $\mathcal{W}$ of the form

$$
\mathcal{W}=\mathcal{W}^{B} \mathcal{D}_{B}-\frac{1}{2} \mathfrak{W}^{b c} M_{b c}-\mathrm{i} \mathfrak{W} J
$$

The introduction of representation (C.2) is accompanied by the appearance of a new gauge invariance that acts on $\mathcal{W}$ and $\overline{\mathcal{W}}$ by

$$
\mathrm{e}^{\mathcal{W}^{\prime}}=\mathrm{e}^{\mathcal{W}} \mathrm{e}^{-\bar{\Lambda}}, \quad \mathrm{e}^{\overline{\mathcal{W}}^{\prime}}=\mathrm{e}^{\overline{\mathcal{W}}} \mathrm{e}^{-\Lambda}, \quad \Lambda=\Lambda^{B} \mathcal{D}_{B}+\frac{1}{2} \Lambda^{b c} M_{b c}+\mathrm{i} \Lambda J
$$

This transformation should be accompanied by certain transformations of $\mathcal{F}, \Delta \Omega_{\alpha}{ }^{b c}, \Delta \Phi_{\alpha}$ and their conjugates such that $\mathbb{D}_{\alpha}$ and $\overline{\mathbb{D}}_{\alpha}$ remain unchanged, which leads to some restrictions on the superfield parameters in $\Lambda$. In such a setting, the supergravity gauge transformations (3.5) and (C.1) can be realised in two different incarnations: (i) as "background" transformations; and (ii) as "quantum" transformations. The quantum gauge transformations and the $\Lambda$-transformations (C.3) may be used to choose a quantum chiral representation in which the operators $\overline{\mathbb{D}}_{\alpha}$ and $\mathbb{D}_{\alpha}$ take the form:

$$
\begin{aligned}
& \overline{\mathbb{D}}_{\alpha}=\overline{\mathcal{D}}_{\alpha}+\cdots, \\
& \mathbb{D}_{\alpha}=\mathrm{e}^{-2 \mathrm{i} H}\left(\mathcal{N}_{\alpha}{ }^{\beta} \mathcal{D}_{\beta}+\cdots\right) \mathrm{e}^{2 \mathrm{i} H}, \quad \operatorname{det} \mathcal{N}=1, \quad \mathcal{N} \overline{\mathcal{N}}=\mathbb{1}_{2},
\end{aligned}
$$

where we have introduced the $2 \times 2$ matrix $\mathcal{N}=\left(\mathcal{N}_{\alpha}^{\beta}\right)$, its complex conjugate $\overline{\mathcal{N}}$, as well as the differential operator

$$
H=H^{a} \mathcal{D}_{a}, \quad \bar{H}^{a}=H^{a} .
$$

The ellipses in (C.4) denote all terms with the Lorentz and $\mathrm{U}(1)_{R}$ generators. The above steps are analogous to the background-quantum splitting in $4 \mathrm{D} \mathcal{N}=1$ supergravity $[74,75]$ described in detail in [45]. The novel feature of the $3 \mathrm{D} \mathcal{N}=2$ case is the appearance of the matrix $\mathcal{N}$, its origin is explained in [68].

All the building blocks in (C.4), as well as the torsion tensors for $\mathbb{D}_{A}$ can be expressed in terms of the gravitational superfield $H^{a}$ by requiring these covariant derivatives to obey the (anti-)commutation relations for the conventional superspace. In this paper we are only interested in explicit expressions for these objects at first-order in $H^{a}$. In this approximation the covariant derivatives (C.4) are

$$
\begin{aligned}
& \mathbb{D}_{\alpha}=\mathcal{D}_{\alpha}-\mathrm{i} \mathcal{D}_{\alpha} H^{\gamma \delta} \mathcal{D}_{\gamma \delta}+N_{\alpha}{ }^{\beta} \mathcal{D}_{\beta}+2 \overline{\mathcal{R}} H_{\alpha}{ }^{\gamma} \overline{\mathcal{D}}_{\gamma}-\frac{1}{2} \boldsymbol{\Omega}_{\alpha}{ }^{\gamma \delta} M_{\gamma \delta}-\mathrm{i} \boldsymbol{\Phi}_{\alpha} J, \\
& \overline{\mathbb{D}}_{\alpha}=\overline{\mathcal{D}}_{\alpha}-\frac{1}{2} \tilde{\boldsymbol{\Omega}}_{\alpha}{ }^{\gamma \delta} M_{\gamma \delta}-\mathrm{i} \tilde{\boldsymbol{\Phi}}_{\alpha} J,
\end{aligned}
$$


where $N_{\alpha}{ }^{\beta}$ is traceless and is related to $\mathcal{N}_{\alpha}{ }^{\beta}$ as follows:

$$
\mathcal{N}_{\alpha}^{\beta}-N_{\alpha}{ }^{\beta}=\delta_{\alpha}^{\beta}+H_{\alpha}{ }^{\gamma} \mathcal{C}_{\gamma}^{\beta}+H^{\beta \gamma} \mathcal{C}_{\alpha \gamma}+2 \mathrm{i} H_{\alpha}{ }^{\beta} \mathcal{S} .
$$

All the superfields $N_{\alpha}^{\beta}, \boldsymbol{\Omega}_{\alpha}^{\gamma \delta}, \tilde{\boldsymbol{\Omega}}_{\alpha}^{\gamma \delta}, \boldsymbol{\Phi}_{\alpha}$ and $\tilde{\boldsymbol{\Phi}}_{\alpha}$ may be expressed in term of $H^{a}$ and its covariant derivatives. These are fixed by requiring the following algebra to be satisfied:

$$
\begin{aligned}
& \left\{\mathbb{D}_{\alpha}, \mathbb{D}_{\beta}\right\}=-4 \overline{\mathbb{R}} M_{\alpha \beta}, \quad\left\{\overline{\mathbb{D}}_{\alpha}, \overline{\mathbb{D}}_{\beta}\right\}=4 \mathbb{R} M_{\alpha \beta}, \\
& \left\{\mathbb{D}_{\alpha}, \overline{\mathbb{D}}_{\beta}\right\}=-2 \mathrm{i} \mathbb{D}_{\alpha \beta}-2 \mathbb{C}_{\alpha \beta} J-4 \mathrm{i} \varepsilon_{\alpha \beta} \mathbb{S} J+4 \mathrm{i} \mathbb{S} M_{\alpha \beta}-2 \varepsilon_{\alpha \beta} \mathbb{C}^{\gamma \delta} M_{\gamma \delta} .
\end{aligned}
$$

Here we have defined the deformed vector derivative by

$$
\mathbb{D}_{a}=-\frac{\mathrm{i}}{4}\left(\gamma_{a}\right)^{\alpha \beta}\left\{\mathbb{D}_{(\alpha}, \overline{\mathbb{D}}_{\beta)}\right\}+\mathrm{i} \mathbb{C}_{a} J+2 \mathbb{S} M_{a} .
$$

It should be noted that in the chiral representation the torsion superfields $\mathbb{C}_{a}$ and $\mathbb{S}$ are no longer real; instead they obey some modified reality conditions. Similarly, $\overline{\mathbb{R}}$ is no longer conjugate to $\mathbb{R}$. Direct calculations lead to the following expressions:

$$
\begin{aligned}
N_{\alpha}{ }^{\beta} & =-\frac{1}{2} \overline{\mathcal{D}}_{\gamma} \mathcal{D}^{\gamma} H_{\alpha}{ }^{\beta}, \\
\boldsymbol{\Omega}_{\alpha, \beta \gamma} & =4\left(\mathcal{D}_{\alpha} H_{(\beta}{ }^{\delta}\right) \mathcal{C}_{\gamma) \delta}+4 \mathrm{i} \mathcal{S} \mathcal{D}_{\alpha} H_{\beta \gamma}+2 \mathcal{D}_{\alpha} N_{\beta \gamma}-4 \mathrm{i} \varepsilon_{\alpha(\beta} \Phi_{\gamma)}, \\
\tilde{\boldsymbol{\Omega}}_{\alpha, \beta \gamma} & =4 \mathrm{i} \varepsilon_{\alpha(\beta} \tilde{\Phi}_{\gamma)}, \\
\boldsymbol{\Phi}_{\alpha} & =\frac{\mathrm{i}}{4} \mathcal{D}_{\alpha} \mathcal{D}^{\beta} \overline{\mathcal{D}}^{\gamma} H_{\beta \gamma}+\mathrm{i}\left(\mathcal{D}_{\alpha} \mathcal{C}_{\beta \gamma}\right) H^{\beta \gamma}+4\left(\mathcal{D}^{\beta} \mathcal{S}\right) H_{\alpha \beta}, \\
\tilde{\mathbf{\Phi}}_{\alpha} & =\frac{\mathrm{i}}{4} \overline{\mathcal{D}}_{\alpha} \overline{\mathcal{D}}^{\beta} \mathcal{D}^{\gamma} H_{\beta \gamma} .
\end{aligned}
$$

The deformed torsion superfields are

$$
\begin{aligned}
\mathbb{R}= & \mathcal{R}+\frac{\mathrm{i}}{2} \overline{\mathcal{D}}^{\alpha} \tilde{\boldsymbol{\Phi}}_{\alpha} \\
\overline{\mathbb{R}}= & \overline{\mathcal{R}}+\frac{\mathrm{i}}{2} \mathcal{C}^{\alpha \beta \gamma} \mathcal{D}_{\alpha} H_{\beta \gamma}+\frac{4 \mathrm{i}}{3}\left(\mathcal{D}_{\beta} \mathcal{S}\right) \mathcal{D}_{\alpha} H^{\alpha \beta}-\frac{1}{6}\left(\overline{\mathcal{D}}_{\alpha} \overline{\mathcal{R}}\right) \mathcal{D}_{\beta} H^{\alpha \beta} \\
& +2 \overline{\mathcal{R}} \mathcal{C}^{\alpha \beta} H_{\alpha \beta}+\frac{\mathrm{i}}{2} \mathcal{D}^{\alpha} \boldsymbol{\Phi}_{\alpha} \\
\mathbb{S}= & \mathcal{S}-\frac{1}{8} \overline{\mathcal{C}}^{\alpha \beta \gamma} \mathcal{D}_{\alpha} H_{\beta \gamma}-\frac{1}{24}\left(8 \overline{\mathcal{D}}_{\alpha} \mathcal{S}-\mathrm{i} \mathcal{D}_{\alpha} \mathcal{R}\right) \mathcal{D}_{\beta} H^{\alpha \beta}-\frac{\mathrm{i}}{4} N^{\alpha \beta} \mathcal{C}_{\alpha \beta} \\
& +\frac{1}{8} \mathcal{D}^{\alpha} \tilde{\boldsymbol{\Phi}}_{\alpha}-\frac{1}{8} \overline{\mathcal{D}}^{\alpha} \boldsymbol{\Phi}_{\alpha} \\
\mathbb{C}_{\alpha \beta}= & \mathcal{C}_{\alpha \beta}-\frac{1}{4} \overline{\mathcal{D}}^{\gamma} \mathcal{D}_{\gamma} N_{\alpha \beta}-2 \mathrm{i} \mathcal{S} N_{\alpha \beta}-2 H_{\alpha \beta} \mathcal{R} \overline{\mathcal{R}}+\frac{\mathrm{i}}{2} \mathcal{D}_{(\alpha} \tilde{\mathbf{\Phi}}_{\beta)}+\frac{\mathrm{i}}{2} \overline{\mathcal{D}}_{(\alpha} \boldsymbol{\Phi}_{\beta)}-N_{(\alpha}{ }^{\delta} \mathcal{C}_{\beta) \delta} \\
& -\frac{\mathrm{i}}{2}\left(\mathcal{D}_{\gamma} H^{\gamma \delta}\right) \overline{\mathcal{C}}_{\alpha \beta \delta}+\frac{\mathrm{i}}{2}\left(\mathcal{D}^{\gamma} H^{\delta}{ }_{(\alpha}\right) \overline{\mathcal{C}}_{\beta) \gamma \delta}+\frac{\mathrm{i}}{12}\left(5 \mathrm{i} \mathcal{D}^{\gamma} \mathcal{R}-4 \overline{\mathcal{D}}^{\gamma} \mathcal{S}\right) \mathcal{D}_{(\alpha} H_{\beta) \gamma} \\
& +\frac{\mathrm{i}}{12}\left(\mathrm{i} \mathcal{D}_{(\alpha} \mathcal{R}+4 \overline{\mathcal{D}}_{(\alpha} \mathcal{S}\right) \mathcal{D}^{\gamma} H_{\beta) \gamma}-\frac{\mathrm{i}}{12}\left(\mathrm{i} \mathcal{D}^{\gamma} \mathcal{R}+4 \overline{\mathcal{D}}^{\gamma} \mathcal{S}\right) \mathcal{D}_{\gamma} H_{\alpha \beta} .
\end{aligned}
$$

In conclusion, a few comments are in order regarding the chiral representation used above. In order to switch from the original real to the chiral representation, every scalar superfield $T$ has to be transformed to

$$
\mathbb{T}=\mathrm{e}^{-\mathrm{i} H^{a} \mathcal{D}_{a}} T .
$$


This tells us that $\delta T=-\mathrm{i} H^{a} \mathcal{D}_{a} T=\mathbb{T}-T+\mathcal{O}\left(H^{2}\right)$ is the complete variation in the case that $T$ is unconstrained. For instance, the prepotential $\mathcal{V}$ for the $\mathcal{N}=2$ linear multiplet varies as

$$
\delta \mathcal{V}=-\mathrm{i} H^{a} \mathcal{D}_{a} \mathcal{V}
$$

However constrained superfields transform in a more complicated fashion since their constraints must be preserved under shifts in the prepotential. For instance, for a covariantly chiral superfield $\Psi$ of $\mathrm{U}(1)_{R}$ charge $-\frac{1}{2}$ and its conjugate $\bar{\Psi}$ we obtain

$$
\begin{aligned}
& \delta \Psi=-\frac{1}{16} \Psi\left(\left[\mathcal{D}^{\alpha}, \overline{\mathcal{D}}^{\beta}\right] H_{\alpha \beta}-4 \mathrm{i} \mathcal{D}^{a} H_{a}\right) \\
& \delta \bar{\Psi}=-\frac{1}{16} \bar{\Psi}\left(\left[\mathcal{D}^{\alpha}, \overline{\mathcal{D}}^{\beta}\right] H_{\alpha \beta}+4 \mathrm{i} \mathcal{D}^{a} H_{a}\right)-2 \mathrm{i} H^{a} \mathcal{D}_{a} \bar{\Psi}
\end{aligned}
$$

Similarly, it can be shown that the $\mathcal{N}=2$ linear superfield varies as

$$
\begin{aligned}
\delta G= & \frac{\mathrm{i}}{4}\left(\overline{\mathcal{D}}^{\alpha} \mathcal{D}_{\alpha} H^{\gamma \delta}\right)\left[\mathcal{D}_{\gamma}, \overline{\mathcal{D}}_{\delta}\right] \mathcal{V}-\frac{1}{2} H^{\gamma \delta} \overline{\mathcal{D}}^{\alpha} \mathcal{D}_{\alpha} \mathcal{D}_{\gamma \delta} \mathcal{V}-\frac{1}{2}\left(\overline{\mathcal{D}}^{\alpha} H^{\gamma \delta}\right) \mathcal{D}_{\alpha} \mathcal{D}_{\gamma \delta} \mathcal{V} \\
& +\frac{1}{2}\left(\mathcal{D}^{\alpha} H^{\gamma \delta}\right) \overline{\mathcal{D}}_{\alpha} \mathcal{D}_{\gamma \delta} \mathcal{V}-2 \mathrm{i}\left(\overline{\mathcal{D}}_{\alpha} H^{\alpha \gamma}\right) \overline{\mathcal{R}}_{\overline{\mathcal{D}}} \mathcal{V}-2 \mathrm{i}\left(\mathcal{D}^{\alpha} H_{\alpha \gamma}\right) \mathcal{R} \mathcal{D}^{\gamma} \mathcal{V} \\
& -2 \mathrm{i} H^{\alpha \beta}\left(\overline{\mathcal{D}}_{\alpha} \overline{\mathcal{R}}\right) \overline{\mathcal{D}}_{\beta} \mathcal{V}
\end{aligned}
$$

which can be rewritten in the following form:

$$
\begin{aligned}
\delta G= & -\mathrm{i} H^{a} \mathcal{D}_{a} G+\frac{\mathrm{i}}{8}\left(\left[\mathcal{D}^{\alpha}, \overline{\mathcal{D}}_{\alpha}\right] H^{\gamma \delta}\right)\left[\mathcal{D}_{\gamma}, \overline{\mathcal{D}}_{\delta}\right] \mathcal{V} \\
& +H^{\alpha \beta}\left(2 \mathcal{C}_{(\alpha}{ }^{\gamma} \mathcal{D}_{\beta) \gamma}-\mathrm{i}\left(\mathcal{D}_{(\alpha} \mathcal{R}\right) \mathcal{D}_{\beta)}-\mathrm{i}\left(\overline{\mathcal{D}}_{(\alpha} \overline{\mathcal{R}}\right) \overline{\mathcal{D}}_{\beta)}\right) \mathcal{V} \\
& +\frac{1}{2}\left(\mathcal{D}^{\gamma} H^{\alpha \beta}\right)\left(\mathcal{D}_{\alpha \beta} \overline{\mathcal{D}}_{\gamma}+\mathrm{i} \mathcal{C}_{\gamma \alpha} \overline{\mathcal{D}}_{\beta}\right) \mathcal{V}-\frac{1}{2}\left(\overline{\mathcal{D}}^{\gamma} H^{\alpha \beta}\right)\left(\mathcal{D}_{\alpha \beta} \mathcal{D}_{\gamma}-\mathrm{i} \mathcal{C}_{\gamma \alpha} \mathcal{D}_{\beta}\right) \mathcal{V} \\
& +\left(\overline{\mathcal{D}}_{\alpha} H^{\alpha \beta}\right)\left(\frac{\mathrm{i}}{2} \mathcal{C}_{\beta \delta} \mathcal{D}^{\delta}+\mathcal{S} \mathcal{D}_{\beta}-\mathrm{i} \bar{R} \overline{\mathcal{D}}_{\beta}\right) \mathcal{V} \\
& +\left(\mathcal{D}_{\alpha} H^{\alpha \beta}\right)\left(\frac{\mathrm{i}}{2} \mathcal{C}_{\beta \delta} \overline{\mathcal{D}}^{\delta}-\mathcal{S D}_{\beta}-\mathrm{i} R \mathcal{D}_{\beta}\right) \mathcal{V} .
\end{aligned}
$$

Open Access. This article is distributed under the terms of the Creative Commons Attribution License (CC-BY 4.0), which permits any use, distribution and reproduction in any medium, provided the original author(s) and source are credited.

\section{References}

[1] R. Utiyama and B.S. DeWitt, Renormalization of a classical gravitational field interacting with quantized matter fields, J. Math. Phys. 3 (1962) 608 [inSPIRE].

[2] B.S. DeWitt, Dynamical Theory of Groups and Fields, Gordon and Breach, New York, (1965).

[3] K.S. Stelle, Renormalization of Higher Derivative Quantum Gravity, Phys. Rev. D 16 (1977) 953 [INSPIRE]. 
[4] A.A. Starobinsky, A New Type of Isotropic Cosmological Models Without Singularity, Phys. Lett. B 91 (1980) 99 [INSPIRE].

[5] S. Deser, R. Jackiw and S. Templeton, Topologically Massive Gauge Theories, Annals Phys. 140 (1982) 372 [Erratum ibid. 185 (1988) 406] [INSPIRE].

[6] E.A. Bergshoeff, O. Hohm and P.K. Townsend, Massive Gravity in Three Dimensions, Phys. Rev. Lett. 102 (2009) 201301 [arXiv:0901.1766] [INSPIRE].

[7] E.A. Bergshoeff, O. Hohm and P.K. Townsend, More on Massive 3D Gravity, Phys. Rev. D 79 (2009) 124042 [arXiv:0905.1259] [INSPIRE].

[8] M. Nakasone and I. Oda, On Unitarity of Massive Gravity in Three Dimensions, Prog. Theor. Phys. 121 (2009) 1389 [arXiv:0902.3531] [InSPIRE].

[9] S. Deser, Ghost-free, finite, fourth order $D=3$ (alas) gravity, Phys. Rev. Lett. 103 (2009) 101302 [arXiv:0904.4473] [INSPIRE].

[10] N. Ohta, A Complete Classification of Higher Derivative Gravity in 3D and Criticality in 4D, Class. Quant. Grav. 29 (2012) 015002 [arXiv:1109.4458] [INSPIRE].

[11] I. Oda, Renormalizability of Massive Gravity in Three Dimensions, JHEP 05 (2009) 064 [arXiv: 0904.2833] [INSPIRE].

[12] K. Muneyuki and N. Ohta, Unitarity versus Renormalizability of Higher Derivative Gravity in 3D, Phys. Rev. D 85 (2012) 101501 [arXiv:1201.2058] [INSPIRE].

[13] S. Deser, Cosmological topological supergravity, in Quantum Theory of Gravity, S.M. Christensen ed., Adam Hilger, Bristol, (1984), pg. 374-381, [INSPIRE].

[14] W. Li, W. Song and A. Strominger, Chiral Gravity in Three Dimensions, JHEP 04 (2008) 082 [arXiv:0801.4566] [INSPIRE].

[15] S. Carlip, S. Deser, A. Waldron and D.K. Wise, Topologically Massive AdS Gravity, Phys. Lett. B 666 (2008) 272 [arXiv:0807.0486] [INSPIRE].

[16] S. Carlip, S. Deser, A. Waldron and D.K. Wise, Cosmological Topologically Massive Gravitons and Photons, Class. Quant. Grav. 26 (2009) 075008 [arXiv: 0803.3998] [INSPIRE].

[17] S. Deser and J.H. Kay, Topologically massive supergravity, Phys. Lett. B 120 (1983) 97 [INSPIRE].

[18] S.M. Kuzenko, U. Lindström, M. Roček, I. Sachs and G. Tartaglino-Mazzucchelli, Three-dimensional $\mathcal{N}=2$ supergravity theories: From superspace to components, Phys. Rev. D 89 (2014) 085028 [arXiv:1312.4267] [inSPIRE].

[19] S.M. Kuzenko and J. Novak, Supergravity-matter actions in three dimensions and Chern-Simons terms, JHEP 05 (2014) 093 [arXiv: 1401.2307] [INSPIRE].

[20] R. Andringa, E.A. Bergshoeff, M. de Roo, O. Hohm, E. Sezgin and P.K. Townsend, Massive $3 D$ Supergravity, Class. Quant. Grav. 27 (2010) 025010 [arXiv:0907.4658] [INSPIRE].

[21] E.A. Bergshoeff, O. Hohm, J. Rosseel, E. Sezgin and P.K. Townsend, More on Massive 3D Supergravity, Class. Quant. Grav. 28 (2011) 015002 [arXiv: 1005.3952] [INSPIRE].

[22] E. Bergshoeff and M. Ozkan, 3D Born-Infeld Gravity and Supersymmetry, JHEP 08 (2014) 149 [arXiv: 1405.6212] [INSPIRE].

[23] G. Alkac, L. Basanisi, E.A. Bergshoeff, M. Ozkan and E. Sezgin, Massive $\mathcal{N}=2$ supergravity in three dimensions, JHEP 02 (2015) 125 [arXiv:1412.3118] [INSPIRE]. 
[24] M. Brown and S.J. Gates Jr., Superspace Bianchi Identities and the Supercovariant Derivative, Annals Phys. 122 (1979) 443 [InSPIRE].

[25] W. Siegel, Unextended Superfields in Extended Supersymmetry, Nucl. Phys. B 156 (1979) 135 [inSPIRE].

[26] S.J. Gates Jr., M.T. Grisaru, M. Roček and W. Siegel, Superspace Or One Thousand and One Lessons in Supersymmetry, Front. Phys. 58 (1983) 1 [hep-th/0108200] [InSPIRE].

[27] P.S. Howe and R.W. Tucker, Local Supersymmetry in (2+1)-Dimensions. 1. Supergravity and Differential Forms, J. Math. Phys. 19 (1978) 869 [INSPIRE].

[28] P.S. Howe and R.W. Tucker, Local Supersymmetry in (2+1)-Dimensions. 2. An Action for a Spinning Membrane, J. Math. Phys. 19 (1978) 981 [INSPIRE].

[29] S.M. Kuzenko, U. Lindström and G. Tartaglino-Mazzucchelli, Off-shell supergravity-matter couplings in three dimensions, JHEP 03 (2011) 120 [arXiv:1101.4013] [INSPIRE].

[30] D. Butter, S.M. Kuzenko, J. Novak and G. Tartaglino-Mazzucchelli, Conformal supergravity in three dimensions: New off-shell formulation, JHEP 09 (2013) 072 [arXiv:1305.3132] [INSPIRE].

[31] D. Butter, S.M. Kuzenko, J. Novak and G. Tartaglino-Mazzucchelli, Conformal supergravity in three dimensions: Off-shell actions, JHEP 10 (2013) 073 [arXiv:1306.1205] [INSPIRE].

[32] S.M. Kuzenko, J. Novak and G. Tartaglino-Mazzucchelli, $N=6$ superconformal gravity in three dimensions from superspace, JHEP 01 (2014) 121 [arXiv:1308.5552] [INSPIRE].

[33] S.M. Kuzenko and G. Tartaglino-Mazzucchelli, Three-dimensional $N=2$ (AdS) supergravity and associated supercurrents, JHEP 12 (2011) 052 [arXiv:1109.0496] [INSPIRE].

[34] S. Theisen, Fourth-order supergravity, Nucl. Phys. B 263 (1986) 687 [InSPIRE].

[35] M. Roček and P. van Nieuwenhuizen, $N \geq 2$ supersymmetric Chern-Simons terms as $D=3$ extended conformal supergravity, Class. Quant. Grav. 3 (1986) 43 [INSPIRE].

[36] B.M. Zupnik and D.G. Pak, Superfield Formulation of the Simplest Three-dimensional Gauge Theories and Conformal Supergravities, Theor. Math. Phys. 77 (1988) 1070 [INSPIRE].

[37] H. Nishino and S.J. Gates Jr., Chern-Simons theories with supersymmetries in three-dimensions, Int. J. Mod. Phys. A 8 (1993) 3371 [INSPIRE].

[38] P.S. Howe, J.M. Izquierdo, G. Papadopoulos and P.K. Townsend, New supergravities with central charges and Killing spinors in (2+1)-dimensions, Nucl. Phys. B 467 (1996) 183 [hep-th/9505032] [INSPIRE].

[39] B. de Wit, A.K. Tollsten and H. Nicolai, Locally supersymmetric $D=3$ nonlinear $\sigma$-models, Nucl. Phys. B 392 (1993) 3 [hep-th/9208074] [INSPIRE].

[40] J.M. Izquierdo and P.K. Townsend, Supersymmetric space-times in (2+1) AdS supergravity models, Class. Quant. Grav. 12 (1995) 895 [gr-qc/9501018] [INSPIRE].

[41] N.S. Deger, A. Kaya, E. Sezgin and P. Sundell, Matter coupled Ad $S_{3}$ supergravities and their black strings, Nucl. Phys. B 573 (2000) 275 [hep-th/9908089] [InSPIRE].

[42] J. Wess and B. Zumino, Superfield Lagrangian for Supergravity, Phys. Lett. B 74 (1978) 51 [INSPIRE].

[43] K.S. Stelle and P.C. West, Minimal Auxiliary Fields for Supergravity, Phys. Lett. B 74 (1978) 330 [INSPIRE]. 
[44] S. Ferrara and P. van Nieuwenhuizen, The Auxiliary Fields of Supergravity, Phys. Lett. B 74 (1978) 333 [InSPIRE].

[45] I. L. Buchbinder and S. M. Kuzenko, Ideas and methods of supersymmetry and supergravity: Or a walk through superspace, IOP, Bristol, 1995 (revised edition: 1998), pg. 656, [INSPIRE].

[46] J. Wess and J. Bagger, Supersymmetry and Supergravity, Princeton University Press, Princeton, (1992).

[47] M.F. Sohnius and P.C. West, An Alternative Minimal Off-Shell Version of $N=1$ Supergravity, Phys. Lett. B 105 (1981) 353 [InSPIRE].

[48] P. Breitenlohner, A Geometric Interpretation of Local Supersymmetry, Phys. Lett. B 67 (1977) 49 [INSPIRE].

[49] P. Breitenlohner, Some Invariant Lagrangians for Local Supersymmetry, Nucl. Phys. B 124 (1977) 500 [InSPIRE].

[50] W. Siegel and S.J. Gates Jr., Superfield Supergravity, Nucl. Phys. B 147 (1979) 77 [inSPIRE].

[51] D. Butter and S.M. Kuzenko, A dual formulation of supergravity-matter theories, Nucl. Phys. B 854 (2012) 1 [arXiv:1106.3038] [INSPIRE].

[52] A. Achucarro and P.K. Townsend, A Chern-Simons Action for Three-Dimensional anti-de Sitter Supergravity Theories, Phys. Lett. B 180 (1986) 89 [INSPIRE].

[53] S. Deser, Scale invariance and gravitational coupling, Annals Phys. 59 (1970) 248 [InSPIRE].

[54] B. Zumino, Effective Lagrangians and broken symmetries, in Lectures on Elementary Particles and Quantum Field Theory, Vol. 2, S. Deser, M. Grisaru and H. Pendleton eds., Cambridge, MA, U.S.A (1970), pg. 437-500 [inSPIRE].

[55] B.M. Zupnik and D.G. Pak, Differential and Integral Forms in Supergauge Theories and Supergravity, Class. Quant. Grav. 6 (1989) 723 [InSPIRE].

[56] U. Lindström and M. Roček, A super Weyl invariant spinning membrane, Phys. Lett. B 218 (1989) 207 [INSPIRE].

[57] P. van Nieuwenhuizen, $D=3$ Conformal Supergravity and Chern-Simons Terms, Phys. Rev. D 32 (1985) 872 [INSPIRE].

[58] S.M. Kuzenko and G. Tartaglino-Mazzucchelli, Conformal supergravities as Chern-Simons theories revisited, JHEP 03 (2013) 113 [arXiv: 1212.6852] [INSPIRE].

[59] J. Wess and B. Zumino, The Component Formalism Follows From the Superspace Formulation of Supergravity, Phys. Lett. B 79 (1978) 394 [INSPIRE].

[60] T. Kugo and S. Uehara, $N=1$ Superconformal Tensor Calculus: Multiplets With External Lorentz Indices and Spinor Derivative Operators, Prog. Theor. Phys. 73 (1985) 235 [INSPIRE].

[61] I.L. Buchbinder, S.M. Kuzenko and O.A. Solovev, One loop counterterms of Wess-Zumino model in the $N=1$ nonminimal supergravity background, Nucl. Phys. B 322 (1989) 277 [INSPIRE].

[62] D. Butter, Background field formalism for chiral matter and gauge fields conformally coupled to supergravity, Nucl. Phys. B 828 (2010) 233 [arXiv:0909.4901] [INSPIRE].

[63] D. Butter and J. Novak, Component reduction in $N=2$ supergravity: the vector, tensor and vector-tensor multiplets, JHEP 05 (2012) 115 [arXiv:1201.5431] [INSPIRE]. 
[64] M. Becker, D. Constantin, S.J. Gates Jr., W.D. Linch III, W. Merrell and J. Phillips, $M$ theory on Spin(7) manifolds, fluxes and $3-D, N=1$ supergravity, Nucl. Phys. B 683 (2004) 67 [hep-th/0312040] [INSPIRE].

[65] S.M. Kuzenko, Supersymmetric Spacetimes from Curved Superspace, PoS(CORFU2014) 140 [arXiv: 1504.08114] [INSPIRE].

[66] D. Butter and S.M. Kuzenko, New higher-derivative couplings in $4 D N=2$ supergravity, JHEP 03 (2011) 047 [arXiv: 1012.5153] [INSPIRE].

[67] I.L. Buchbinder, S.M. Kuzenko and A.A. Tseytlin, On low-energy effective actions in $N=2$, $N=4$ superconformal theories in four-dimensions, Phys. Rev. D 62 (2000) 045001 [hep-th/9911221] [INSPIRE].

[68] S.M. Kuzenko, Prepotentials for $N=2$ conformal supergravity in three dimensions, JHEP 12 (2012) 021 [arXiv:1209.3894] [INSPIRE].

[69] S.M. Kuzenko, U. Lindström and G. Tartaglino-Mazzucchelli, Three-dimensional ( $p, q)$ AdS superspaces and matter couplings, JHEP 08 (2012) 024 [arXiv:1205.4622] [INSPIRE].

[70] S.M. Kuzenko and G. Tartaglino-Mazzucchelli, Different representations for the action principle in $4 D N=2$ supergravity, JHEP 04 (2009) 007 [arXiv:0812.3464] [INSPIRE].

[71] M.T. Grisaru and M.E. Wehlau, Superspace measures, invariant actions and component projection formulae for (2,2) supergravity, Nucl. Phys. B 457 (1995) 219 [hep-th/9508139] [INSPIRE].

[72] P.S. Howe, K.S. Stelle and P.K. Townsend, The Vanishing Volume of $N=1$ Superspace, Phys. Lett. B 107 (1981) 420 [INSPIRE].

[73] E.I. Buchbinder, S.M. Kuzenko and I.B. Samsonov, Superconformal field theory in three dimensions: Correlation functions of conserved currents, JHEP 06 (2015) 138 [arXiv: 1503.04961] [INSPIRE].

[74] M.T. Grisaru and W. Siegel, Supergraphity. 1. Background field formalism, Nucl. Phys. B 187 (1981) 149 [inSPIRE].

[75] M.T. Grisaru and W. Siegel, Supergraphity. 2. Manifestly Covariant Rules and Higher Loop Finiteness, Nucl. Phys. B 201 (1982) 292 [Erratum ibid. B 206 (1982) 496] [INSPIRE].

[76] W. Siegel, Solution to Constraints in Wess-Zumino Supergravity Formalism, Nucl. Phys. B 142 (1978) 301 [inSPIRE]. 